\title{
Probabilistic micromechanical spatial variability quantification in laminated composites
}

\author{
S. Naskar ${ }^{\mathrm{a}}$, T. Mukhopadhyay ${ }^{\mathrm{b}}$, S. Sriramula ${ }^{\mathrm{a}}$ \\ ${ }^{a}$ School of Engineering, University of Aberdeen, Aberdeen, UK \\ ${ }^{b}$ Department of Engineering Science, University of Oxford, Oxford, UK \\ Email address: tanmoy.mukhopadhyay@eng.ox.ac.uk (Tanmoy Mukhopadhyay)
}

\begin{abstract}
This article presents a probabilistic framework to characterize the dynamic and stability parameters of composite laminates with spatially varying micro and macro-mechanical system properties. A novel approach of stochastic representative volume element (SRVE) is developed in the context of two dimensional plate-like structures for accounting the correlated spatially varying properties. The physically relevant random field based uncertainty modelling approach with spatial correlation is adopted in this paper on the basis of Karhunen-Loève expansion. An efficient coupled HDMR and DMORPH based stochastic algorithm is developed for composite laminates to quantify the probabilistic characteristics in global responses. Convergence of the algorithm for probabilistic dynamics and stability analysis of the structure is verified and validated with respect to direct Monte Carlo simulation (MCS) based on finite element method. The significance of considering higher buckling modes in a stochastic analysis is highlighted. Sensitivity analysis is performed to ascertain the relative importance of different macromechanical and micromechanical properties. The importance of incorporating source-uncertainty in spatially varying micromechanical material properties is demonstrated numerically. The results reveal that stochasticity (/ system irregularity) in material and structural attributes influences the system performance significantly depending on the type of analysis and the adopted uncertainty modelling approach, affirming the necessity to consider different forms of source-uncertainties during the analysis to ensure adequate safety, sustainability and robustness of the structure.
\end{abstract}

Keywords: composite laminate; micromechanical random field; spatially correlated material properties; stochastic natural frequency; stochastic buckling load; stochastic mode shape 


\section{Contents}

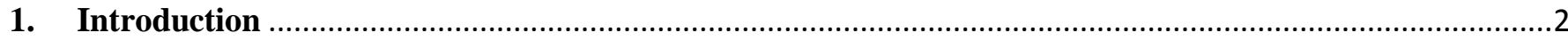

2. Stochastic dynamics and stability analysis of composite plates ..............................................................

3. HDMR based surrogate modelling coupled with DMORPH algorithm ..............................................

4. Stochastic representative volume element based framework for uncertainty quantification ..................12

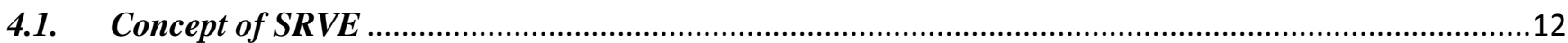

4.2. Characterization of correlated material properties based on Karhunen-Loève expansion ...................14

4.3. Description of the uncertainty quantification framework ......................................................................17

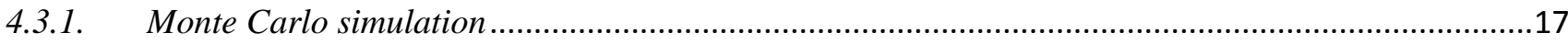

4.3.2. Modelling of source-uncertainty at the input level ........................................................................18

4.3.3. Propagation of uncertainty based on HDMR coupled with DMORPH algorithm ...........................20

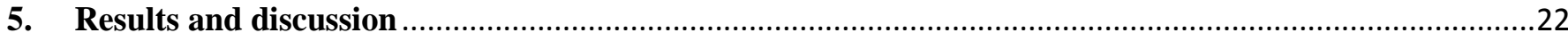

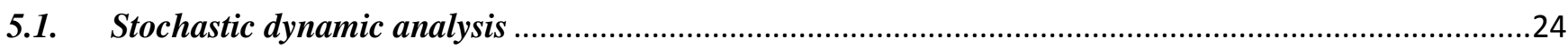

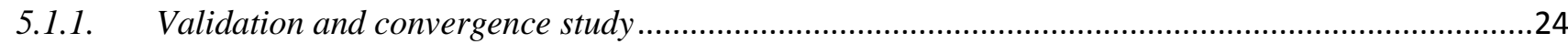

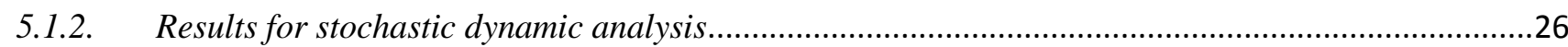

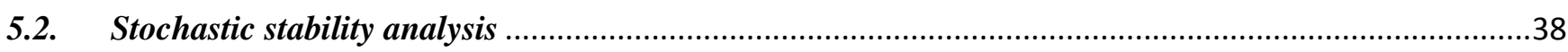

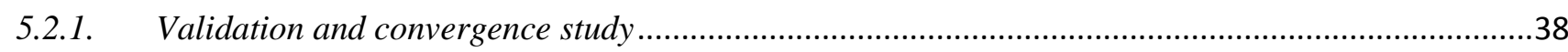

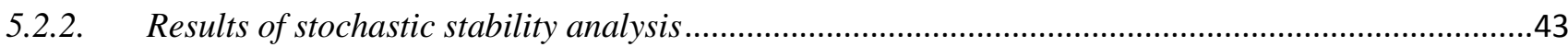

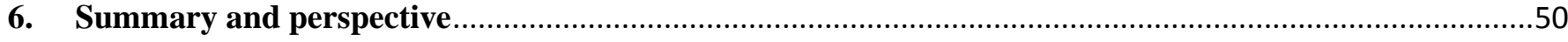

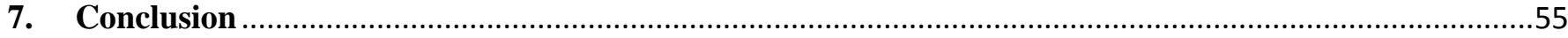

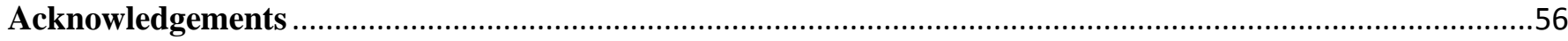

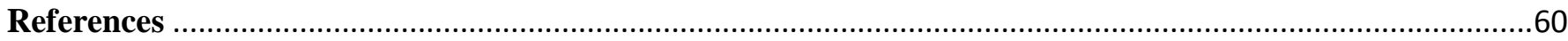

\section{Introduction}

Composite structures are widely used in modern aerospace, construction, marine and automobile applications because of high strength and stiffness with lightweight and tailorable properties. Even though laminated composite structures have the advantage of modulating large number of design parameters to achieve various application-specific requirements, this concurrently brings the challenge of manufacturing the structure according to exact design specifications. Large-scale production of such structures according to the requirements of industry is often subjected to large amount of variability arising from unavoidable manufacturing imperfections (such as intra-laminate voids and excess matrix voids, excess resin between plies, incomplete curing of resin, porosity, variations in lamina thickness and fibre properties), lack of experiences and complexity of the structural configuration. The issue aggravates further due to uncertain 
operational and environmental factors and the possibility of incurring different forms of damages and defects during the service life. In general, uncertainties can be broadly classified into three categories, aleatoric (due to variability in structural parameters), epistemic (due to lack of adequate information about the system) and prejudicial (due to absence of stochasticity characterization of the structural system) (Agarwal et al. (2004), Oberkampf et al. (2001), Sriramula and Chryssanthopoulos (2009), Dey et al. (2018a)). Composite structures being susceptible to multiple forms of uncertainties, the structural performances are often subjected to a significant element of risk. Thus it is of prime importance in case of composite structures to quantify the effect of source-uncertainties so that an inclusive design paradigm could be adopted to avoid any compromise in the aspects of safety and serviceability.

Composite structures have received immense attention from the engineers and scientists concerning their static, dynamic and stability behaviour (Chakrabarti et al. (2011, 2013), Biswal et al. (2016), Dey et al. (2016d), Mandal et al. (2017), Kumari and Behera (2017). Recent studies on the vibration and buckling analysis of advanced lightweight structures (like composites and FGM) in the deterministic regime include non-homogeneity, non-linear behaviour, shear deformation, rotary inertia and effect of elastic foundation in the analysis (Sofiyev et al. (2012, 2017), Sofiyev and Kuruoglu (2014), Haciyev et al. (2018)). Following several decades of deterministic studies, the aspect of considering the effect of uncertainty in material and structural attributes have recently started receiving due attention from the scientific community. Both probabilistic (Sakata et al. (2008), Goyal and Kapania, (2008), Manan and Cooper (2009), Dey et al. (2016a, 2016e, 2018b, 2019), Naskar et al. (2017b), Naskar and Sriramula (2017a, 2017b, 2017c), Naskar (2017)) as well as non-probabilistic (Dey et al. (2016b), Pawar et al. (2012)) approaches have been investigated to analyse the influence of variability in the material and structural attributes of composite structures. Plenty of researches have been reported based on intrusive methods to quantify the uncertainty of composite structures (Lal and Singh (2010), Scarth and Adhikari (2017)), wherein the major drawback can be identified as the requirement of intensive analytical derivation and lack of the ability to obtain complete probabilistic description of the response quantities for systems with spatially varying attributes. A non-intrusive method based on Monte Carlo simulation, as adopted by many researchers (Dey et al. (2016a, 2016g, 2015d), Mukhopadhyay and 

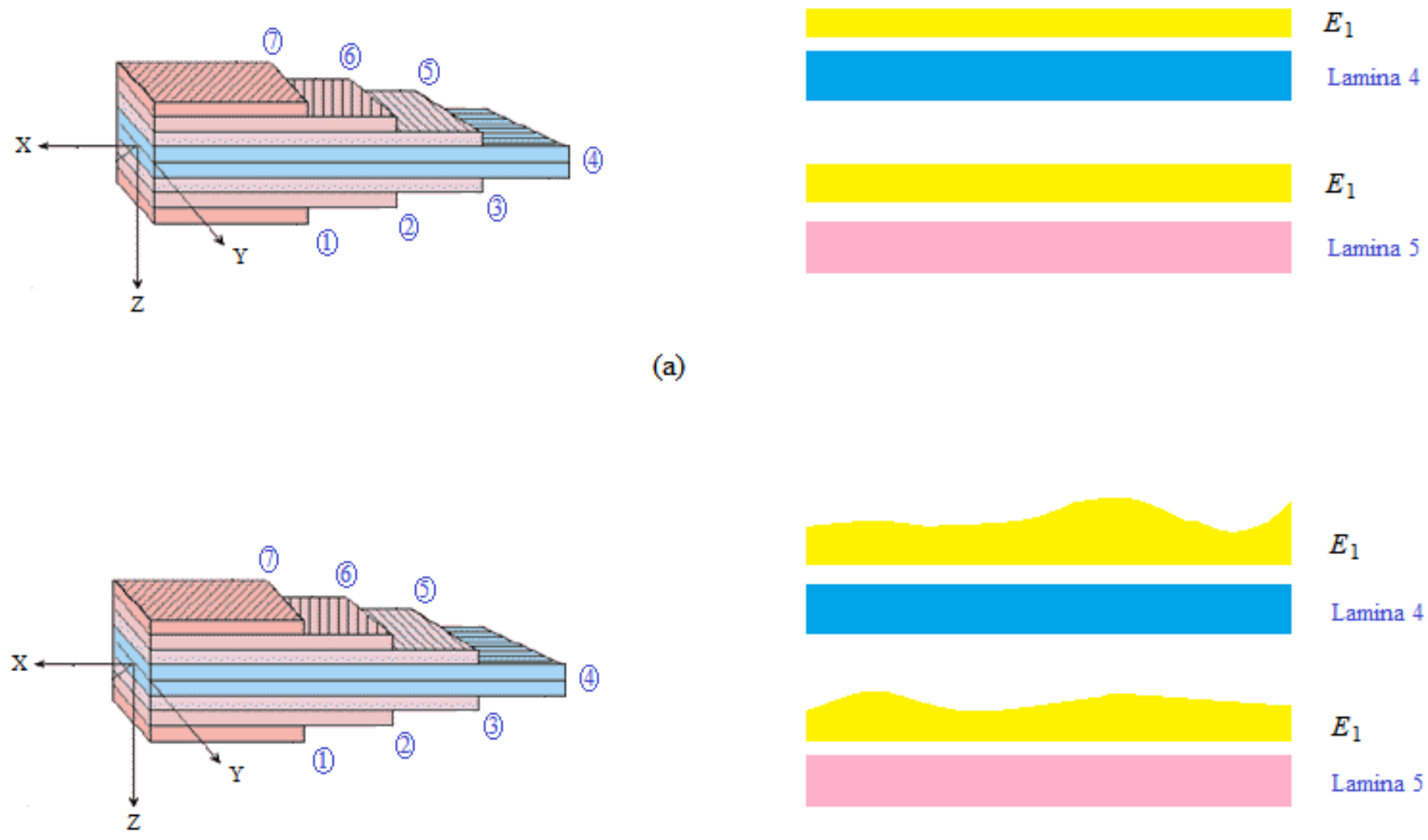

(b)

Fig. 1 (a) Typical distribution of a material property $E_{1}$ along a cross-sectional view (X-Z plane) of two laminae for a random realization in case of the layer-wise random variable approach (b) Typical distribution of a material property $E_{1}$ along a cross-sectional view (X-Z plane) of two laminae for a random realization in case of the random field approach

Adhikari (2016c)), can obtain comprehensive probabilistic descriptions for the response quantities of composite structures. Besides consideration of random variability in material and structural attributes, recent studies related to uncertainty quantification of laminated composite structures include the effect of environmental (Dey et al. (2015a)), operational (Dey et al. (2015b)) and service life conditions (Naskar et al. (2017), Karsh et al. (2018a)) following the non-intrusive approach. A careful consideration of available scientific literature unveils that most of the studies conducted so far to quantify the effect of uncertainty in composite structures are based on a ply-level random variable based approach, where the spatial variation of stochastic parameters in the laminae is neglected. In the previous studies, the material and structural properties of a lamina are assumed constant spatially (i.e. along the $x-y$ plane) for a particular realization (refer to figure 1(a)). Modelling of uncertainty in composite structures based on such random variable based approach is of limited practical resemblance. Therefore, it is essential to consider 
the effect of spatial variability in the material properties (Kazimierz and Kirkner (2001)) of the laminae to quantify the effect of uncertainty accurately.

We aim to quantify the effect of spatially varying lamina properties in this article to characterize the probabilistic descriptions for the dynamics and stability characteristics of composite plates. The aspect of spatial variation of lamina properties is illustrated in figure 1(b) for a random realization (i.e. a typical sample of the Monte Carlo simulation), wherein it can be noticed that the stochastic attributes vary in the $\mathrm{x}-\mathrm{y}$ plane as well as along the $\mathrm{z}$-axis (i.e. for different laminae). Most of the previous investigations in this field have not considered the spatial variation of stochastic attributes as shown in figure 1 (Dey et al. (2016a)). In practical situations, the stochastic attributes often being spatially correlated, it is essential to account for the effect of such correlation to create a physically realistic model of uncertainty. We aim to consider the spatially correlated material attributes in composite laminates based on Karhunen-Loeve expansion (Karhunen (1947), Loève (1977)). However, even after ensuring a physically relevant uncertainty model of composite laminates, as discussed above, the issue of propagation of uncertainty following a computationally viable framework still remains to be addressed. The aspect of computationally efficient uncertainty propagation in context to composite laminates is discussed in the next paragraph.

Uncertainty quantification based on Monte Carlo simulation is a popular approach because of the ability to obtain a comprehensive probabilistic description of the response quantities. However, the major lacuna of this approach is that a Monte Carlo simulation requires thousands of expensive finite element (FE) simulations to be carried out corresponding to the random realizations. Thus, direct Monte Carlo simulation has limited practical use due to the computational intensiveness. To mitigate this lacuna we have developed a surrogate modelling approach based on the high dimensional model representation (HDMR) technique coupled with the diffeomorphic modulation under observable response preserving homotopy (DMORPH) algorithm (Li and Rabitz (2012)) for accounting correlated spatially varying attributes, wherein the uncertainty propagation can be realized following an efficient mathematical medium. 
In the present analysis, uncertainty of the system properties is considered in the elementary micromechanical level to comprehensively analyse the dynamic and stability characteristics of composite laminates. Thus a probabilistic approach is followed, wherein the effect of uncertainty is included in the elementary micromechanical-level first and then the effects are propagated towards the global responses via an efficient surrogate of the actual finite element model. For this purpose, the idea of stochastic representative volume element (SRVE) is proposed in the context of two-dimensional plate-like structures. This article hereafter is organized as, section 2: governing equations for analysing the stochastic dynamics and stability of composite laminates; section 3: brief description of the surrogate model based on HDMR coupled with the DMORPH algorithm; section 4: description of the SRVE approach of uncertainty quantification considering spatially correlated material properties hinged upon the Karhunen-Loève expansion; section 5: results and discussion demonstrating the influence of spatially varying properties on the global responses of composite laminates; section 6: summary and perspective of the present study in context to the available scientific literature; section 7: conclusion.

\section{Stochastic dynamics and stability analysis of composite plates}

In present article, a laminated composite plate with thickness $h$, length $L$ and width $b$ is analysed as shown in figure 2 and 3. The governing equation for stochastic free vibration analysis of a composite plate without damping can be expressed as (refer to the APPENDIX for detail formulation)

$$
[M(\bar{\omega})]\{\ddot{\delta}(\bar{\omega})\}+[K(\bar{\omega})]\{\delta(\bar{\omega})\}=0
$$

where $[K(\bar{\omega})]=\left[K_{e}(\bar{\omega})\right]+\left[K_{\sigma e}(\bar{\omega})\right]$. In the finite element formulation of this study, an eight noded element is considered, wherein each node has five degrees of freedom (two rotations and three translations). The natural frequencies $\left(\omega_{k}\right)$ and mode shapes $\left(S_{k}^{f}\right)$ of the composite plate are obtained by solving an eigenvalue problem based on QR iteration algorithm (Bathe (1990), Rayleigh (1945))

$$
[K(\bar{\omega})] S_{k}^{f}(\bar{\omega})=\left[\omega_{k}(\bar{\omega})\right]^{2}[M(\bar{\omega})] S_{k}^{f}(\bar{\omega})
$$

where $k=1,2,3, \ldots, n$. The superscript $f$ is used to denote the frequency analysis. Here the orthogonality relationship is satisfied as 


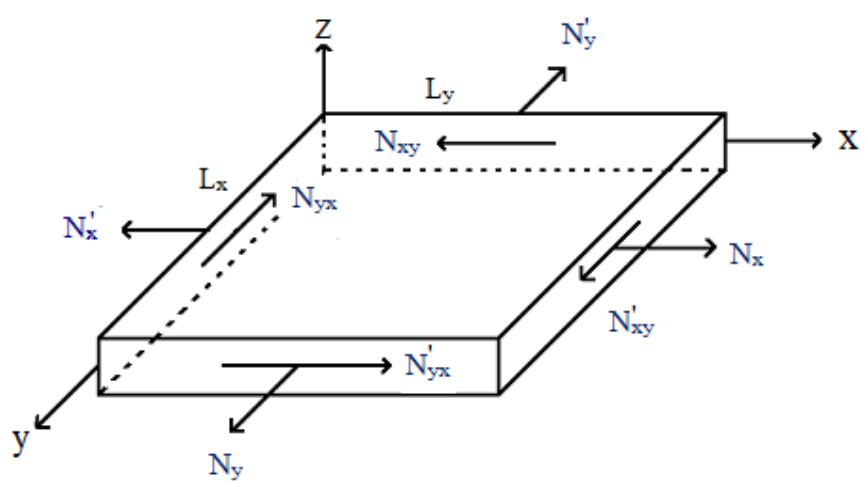

(a)

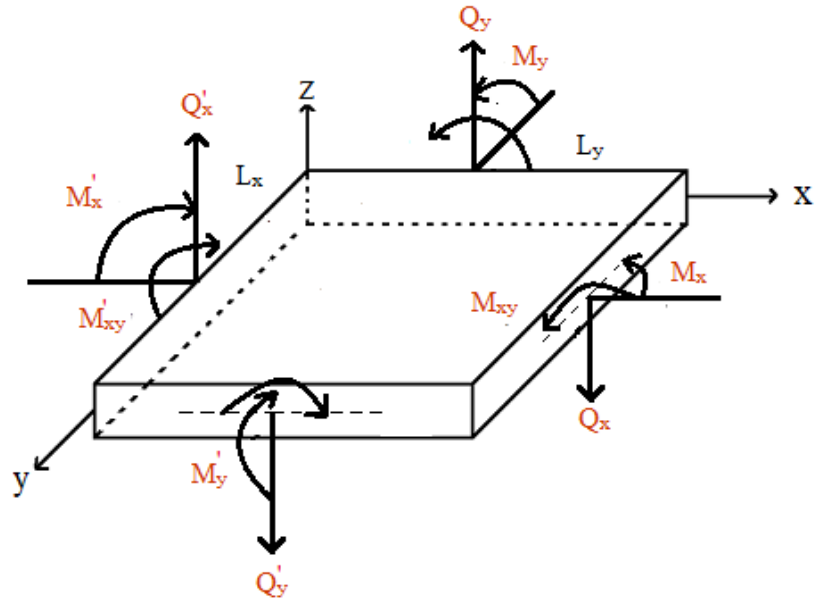

(b)

Fig. 2 (a) Force acting on the plate (b) Moment acting on the plate

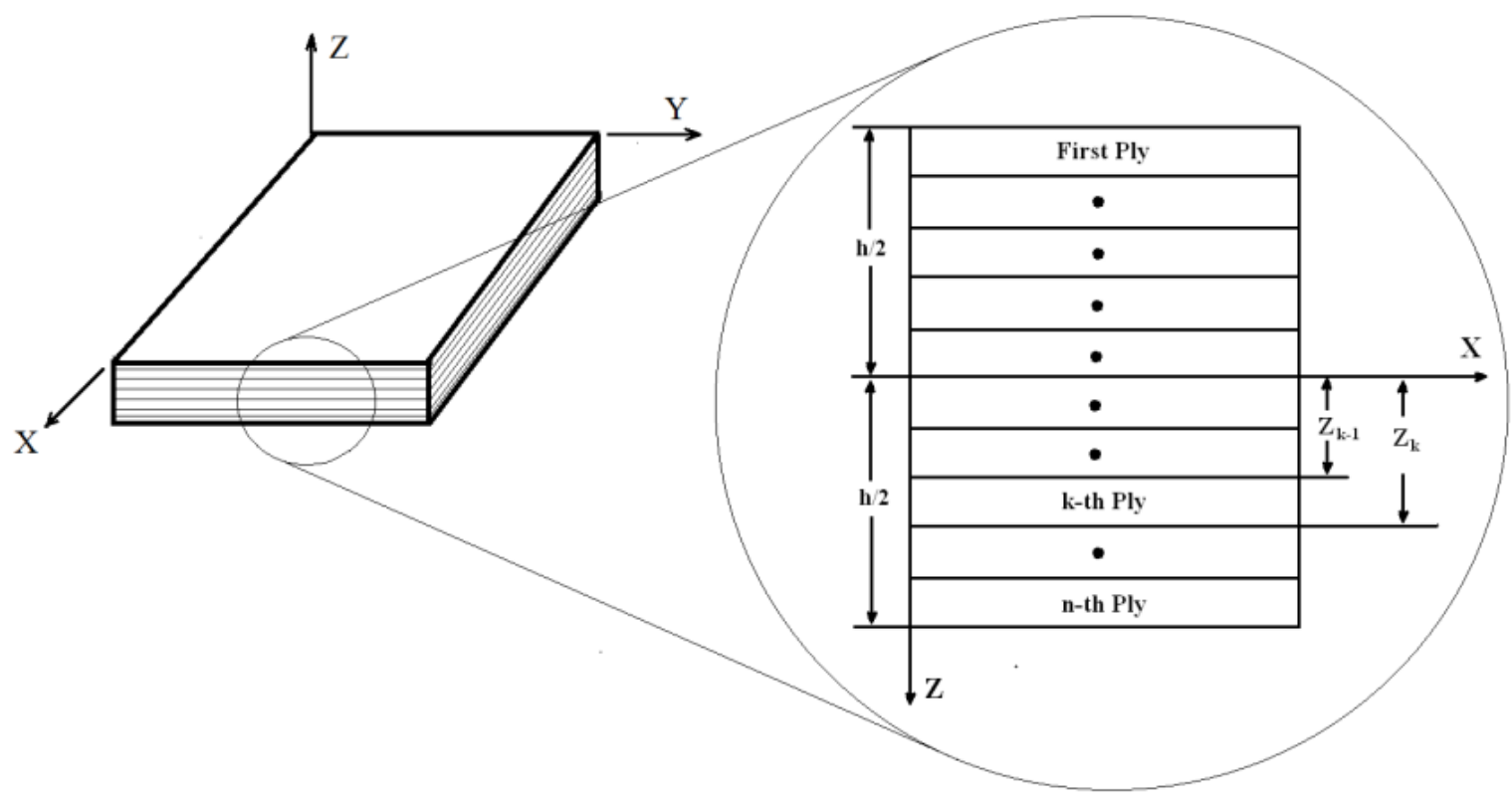

Fig. 3 Laminated composite plate with layer number

$$
\left[S_{i}^{f}(\bar{\omega})\right]^{T}[M(\bar{\omega})] S_{k}^{f}(\bar{\omega})=\bar{\delta}_{i k} \text { and }\left[S_{i}^{f}(\bar{\omega})\right]^{T}[K(\bar{\omega})] S_{k}^{f}(\bar{\omega})=\left[\omega_{k}(\bar{\omega})\right]^{2} \bar{\delta}_{i k}
$$

where $i, k=1,2,3, \ldots . n$ and the Kronecker delta functions $\bar{\delta}_{i k}=0$ for $i \neq k ; \bar{\delta}_{i k}=1$ for $i=k$. The problem of stability analysis is solved through another eigenvalue problem as:

$$
\left[K_{e}(\bar{\omega})\right] S_{k}^{b}=\lambda^{b}(\bar{\omega})\left[K_{\sigma e}(\bar{\omega})\right] S_{k}^{b}(\bar{\omega})
$$

where $\lambda^{b}(\bar{\omega})$ is the stochastic buckling load factor and $S_{k}^{b}$ gives the buckling modeshapes. The superscript $b$ is used to denote the buckling analysis. 


\section{HDMR based surrogate modelling coupled with DMORPH algorithm}

In this section, a brief overview is given for the surrogate modelling approach on the basis of high dimensional model representation (HDMR) coupled with the diffeomorphic modulation under observable response preserving homotopy (DMORPH) algorithm. In general, the surrogate models (Dey et al. (2016a, 2016f, 2018, 2015e), Mukhopadhyay (2019), Mukhopadhyay et al. (2016b, 2016c), Karsh et al. (2018b), Maharshi et al. (2018), Mahata et al. (2016), Metya et al. (2017)) are employed to reduce the number of function evaluations based on actual simulation/ experimental models in a Monte Carlo simulation (refer to figure 4) or a process involving iterative simulations (such as optimization), which need large number of realizations corresponding to random set of input parameters. The surrogate models can encompass any prospective combination of all the input variables within the analysis domain. Thousands of sets of the design input parameters can be generated and pseudo analyses for each set can be efficiently executed by adopting the corresponding surrogate based prediction models. The development of surrogate models is performed in three typical steps: selection of optimal sample points (which are able to collect information of the whole design space) to construct surrogate model, evaluation of responses (i.e. output) corresponding to each of the sample points and formulation of the mathematical/ statistical prediction model to obtain an efficient input-output relationship based on the sample set (containing a set of input parameters and corresponding output parameters).

The present HDMR (Dey et al. (2015c, 2016c, 2017) Mukhopadhyay et al. (2015, 2016a)) based surrogate modelling algorithm is particularly suitable for high dimensional systems (i.e. large number of input parameters) and correlated system properties. The HDMR can form an efficient model to predict the random output responses (e.g. natural frequency and buckling load) in the stochastic analysis domain. This approach is able to treat both independent as well as correlated input variables. The function of DMORPH here is to verify the component function orthogonality following a hierarchical approach. The present formulation decomposes function $\lambda(S)$ with the component functions by input parameters, $S=\left(S_{1}, S_{2}, \ldots, S_{k k}\right)$. As the input parameters are considered to be independent in nature, the component functions can be projected by vanishing condition. In the present analysis, the component functions is portrayed, wherein a unified framework for general HDMR dealing with both correlated as well as 


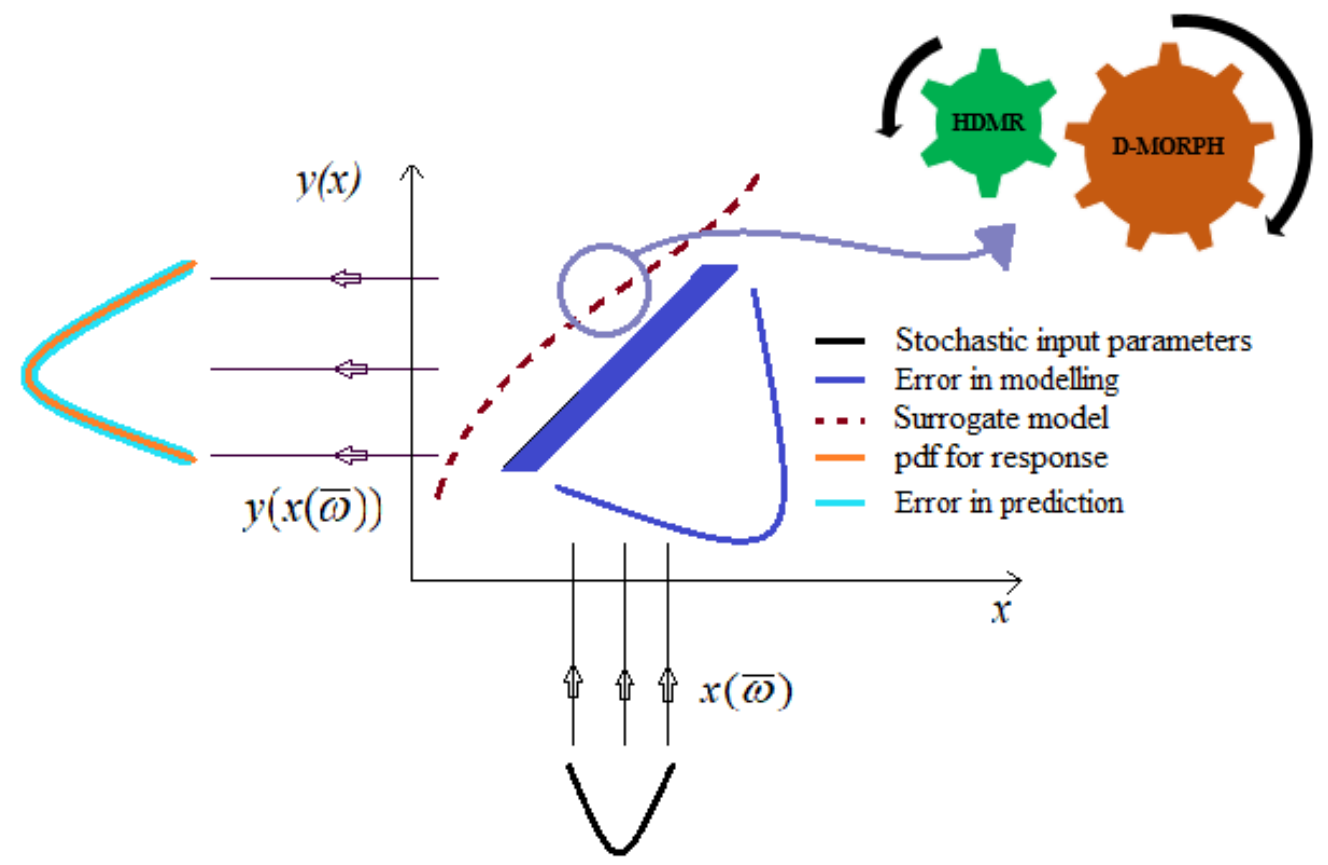

Fig. 4 Surrogate based analysis of stochastic system (Here $x(\bar{\omega})$ and $y(x(\bar{\omega})$ ) are the symbolic representation of stochastic input parameters and output responses respectively. $\bar{\omega}$ denotes the stochasticity of parameters.)

independent variables is developed. For various stochastic input parameters, the output quantity is calculated as follows (Li and Rabitz (2012), Li et al. (2002a, 2002b))

$$
\begin{gathered}
\lambda(S)=\lambda_{0}+\sum_{i=1}^{k k} \lambda_{i}\left(S_{i}\right)+\sum_{1 \leq i<j \leq k k} \lambda_{i j}\left(S_{i}, S_{j}\right)+\ldots \ldots+\lambda_{12 \ldots \ldots k}\left(S_{1}, S_{2}, \ldots ., S_{k k}\right) \\
\lambda(S)=\sum_{u \subseteq k k} \lambda_{u}\left(S_{u}\right)
\end{gathered}
$$

where $\lambda_{0}$ is the zero-th order component function, which represents the mean value. $\lambda_{i}\left(S_{i}\right)$ and $\lambda_{i j}\left(S_{i}, S_{j}\right)$ represent first and second order component functions, respectively. The expression $\lambda_{12 \ldots \ldots k}\left(S_{1}, S_{2}, \ldots, S_{k k}\right)$ is the residual contribution by input parameters. Here $u \subseteq\{1,2, \ldots, k k\}$ indicates the subset wherein $u \subseteq k k$. Note that $u$ includes $\Gamma(\Gamma \in u)$, which is an empty set. According to Hooker's definition, the correlated variables can be expressed as

$$
\left\{\lambda_{u}\left(S_{u} \mid u \subseteq k k\right)\right\}=\operatorname{Arg} \min _{\left\{g_{u} \in L^{2}\left(R^{u}\right), u \subseteq k k\right\}} \int\left(\sum_{u \subseteq k} g_{u}\left(S_{u}\right)-\lambda(S)\right)^{2} w(S) d S
$$




$$
\begin{gathered}
\forall u \subseteq k k, \quad \forall i \in u \quad, \quad \int \lambda_{u}\left(S_{u}\right) w(S) d S_{i} d S_{-u}=0 \\
\forall v \subset u, \quad \forall g_{v}: \int \lambda_{u}\left(S_{u}\right) g_{v}\left(S_{v}\right) w(S) d S=\left\langle\lambda_{u}\left(S_{u}\right), g_{v}\left(S_{v}\right)\right\rangle=0
\end{gathered}
$$

The parameter $\lambda(S)$ is obtained from the design points. Assuming $H$ to be a Hilbert space on the basis of $\left\{h_{1}^{\prime}, h_{2}^{\prime}, \ldots, h_{k k}^{\prime}\right\}$, the bigger subspace $\bar{H}^{\prime}\left(\supset \boldsymbol{H}^{\prime}\right)$ can be expanded by the extended basis $\left\{h_{1}^{\prime}, h_{2}^{\prime}, \ldots\right.$, $\left.h_{k k}^{\prime}, h_{k k+1}^{\prime}, \ldots, h_{m}^{\prime}\right\}$. Then the subspace $\bar{H}^{\prime}$ is decomposed as

$$
\bar{H}^{\prime}=H^{\prime} \oplus H^{\prime \perp}
$$

where $H^{\prime \perp}$ represents the orthogonal complement subspace of $\boldsymbol{H}^{\prime}$ within the subspace $\overline{\boldsymbol{H}}^{\prime}$. Component functions of a second order HDMR expansion can be obtained from basis functions $\{\varphi\}$ (Li et al. (2006)),

$$
\begin{gathered}
\lambda_{i}\left(S_{i}\right) \approx \sum_{r=1}^{k k} \alpha_{r}^{(0) i} \varphi_{r}^{i}\left(S_{i}\right) \\
\lambda_{i j}\left(S_{i}, S_{j}\right) \approx \sum_{r=1}^{k k}\left[\alpha_{r}^{(i j) i} \varphi_{r}^{i}\left(S_{i}\right)+\alpha_{r}^{(i j) j} \varphi_{r}^{j}\left(S_{j}\right)\right]+\sum_{p=1}^{l} \sum_{q=1}^{l} \beta_{p q}^{(0) i j} \varphi_{p}^{i}\left(S_{i}\right) \varphi_{q}^{j}\left(S_{j}\right)
\end{gathered}
$$

The HDMR based expansions for $N_{\text {samp }}$ sample points of $S$ is represented by a linear system of algebraic equations

$$
\Gamma J=\hat{R}
$$

where $\Gamma$ represents a matrix $\left(N_{\text {samp }} \times \tilde{t}\right)$, where the elements are basis functions at $N_{\text {samp }}$ values of $S ; J$ denotes a vector having $\tilde{t}$ dimension of all the unknown combination coefficients; $\hat{R}$ is a vector having $N_{\text {samp }}$ dimension, where the $l$-th element is $\lambda\left(S^{(l)}\right)-\lambda_{0} . S^{(l)}$ is the $l$-th sample of $S$, and $\lambda_{0}$ represents the average value of all $\lambda\left(S^{(l)}\right)$. Regression equation for the least squares can be written as

$$
\frac{1}{N_{\text {samp }}} \Gamma^{T} \Gamma J=\frac{1}{N_{\text {samp }}} \Gamma^{T} \hat{R}
$$

Because of using extended bases, some of the rows of the above expression become identical and these can be removed for obtaining an underdetermined algebraic system of equation

$$
A J=\hat{V}
$$


This has many solutions for $J$ composing a manifold $Y \in \mathfrak{R}^{\tilde{t}}$. Thus a solution $J$ from $Y$ is found to force the HDMR component functions that satisfy hierarchical orthogonal condition. DMORPH regression can provide a solution for ensuring the additional condition

$$
\frac{d J(l)}{d l}=\chi v(l)=\left(I_{t}-A^{+} A\right) v(l)
$$

where $\chi$ is an orthogonal projector having the following properties

$$
\begin{gathered}
\chi^{2}=\chi \quad \text { and } \quad \chi^{T}=\chi \\
\chi=\chi^{2}=\chi^{T} \chi
\end{gathered}
$$

The free function vector can be adopted for ensuring the wide domain for $J(l)$ and to reduce the cost $\kappa(J(l))$ simultaneously

$$
v(l)=-\frac{\partial \kappa(J(l))}{\partial J}
$$

Then,

$$
\begin{aligned}
\frac{\partial \kappa(J(l))}{\partial l} & =\left(\frac{\partial \kappa(J(l))}{\partial J}\right)^{T} \frac{\partial J(l)}{\partial l}=\left(\frac{\partial \kappa(J(l))}{\partial J}\right)^{T} P v(l) \\
& =-\left(P \frac{\partial \kappa(J(l))}{\partial J}\right)^{T}\left(P \frac{\partial \kappa(J(l))}{\partial J}\right) \leq 0
\end{aligned}
$$

The cost function is obtained in a quadratic form

$$
\kappa=\frac{1}{2} J^{T} B J
$$

where, $B$ denotes a positive definite symmetric matrix and $J_{\infty}$ is expressed as

$$
J_{\infty}=V_{t}\left(U_{\tilde{t}-r}^{T} V_{\tilde{t}-r}\right)^{-1} U_{\tilde{t}-r}^{T} A^{+} \hat{V}
$$

Here the last columns $(\tilde{t}-r)$ in $U$ and $V$ are obtained as $U_{\tilde{t}-r}$ and $V_{\tilde{t}-r}$ which is obtained by decomposition of $\chi B$

$$
\chi B=U\left[\begin{array}{ll}
\bar{S}_{r} & 0 \\
0 & 0
\end{array}\right] V^{T}
$$

The solution $J_{\infty}$ in $Y$, which is unique, indicates the minimized cost function. Here DMORPH regression is adopted to obtain $J$ ensuring the HDMR component functions' orthogonality in a hierarchical manner. 


\section{Stochastic representative volume element based framework for uncertainty quantification}

\subsection{Concept of SRVE}

In this paper a concept of stochastic representative volume element (SRVE) is proposed for twodimensional plate-like structures to account for the effect of spatial randomness of material properties. According to this approach, each of the representative units (structural element) is considered to be stochastic in nature, instead of considering the homogenized mechanical properties of a conventional representative volume element (RVE) throughout the entire solid domain. As per the traditional approach, one RVE is analysed typically and the assumption is that a single RVE can represent the entire analysis domain (Sriramula and Chryssanthopoulos (2009), Mukhopadhyay et al. (2017a, 2017b, 2018a)). However, this approach of analysis can lead toeroneous outcomes, specially in case of stochastic systems with spatial randomness in material and other attributes. To analyse such systems, it is essential to account for the effect of the distribution of stocahstic mechanical properties along the spatial location of different zones of a plate-like structure.

According to the present approach, the entire plate is assumed to be consisted of a finite number of SRVEs. Thus mechanical properties of a SRVE are dependent on its stochastic material and structural properties. Following this framework, it becomes feasible to consider the spatial randomness in a structural system more realistically. The global responses (such as natural frequencies and buckling loads) of the plate are computed by propagating the mechanical information acquired in the elementary local level (SRVEs) towards the global level by combing (/assembling) the SRVEs applying the principles of solid mechanics (finite element approach in the present study). Recently, a similar concept has been proposed for analyzing hexagonal honeycomb-like lattices having spatial irregularity (Mukhopadhyay and Adhikari (2016a, 2016b, 2017a), Mukhopadhyay et al. (2018a), Mukhopadhyay (2017)), wherein multiple representative unit cell elements (RUCE) are analysed instead of the conventional approach of considering a single unit cell. The entire lattice structure is assumed to be consisted of several RUCEs and the global 


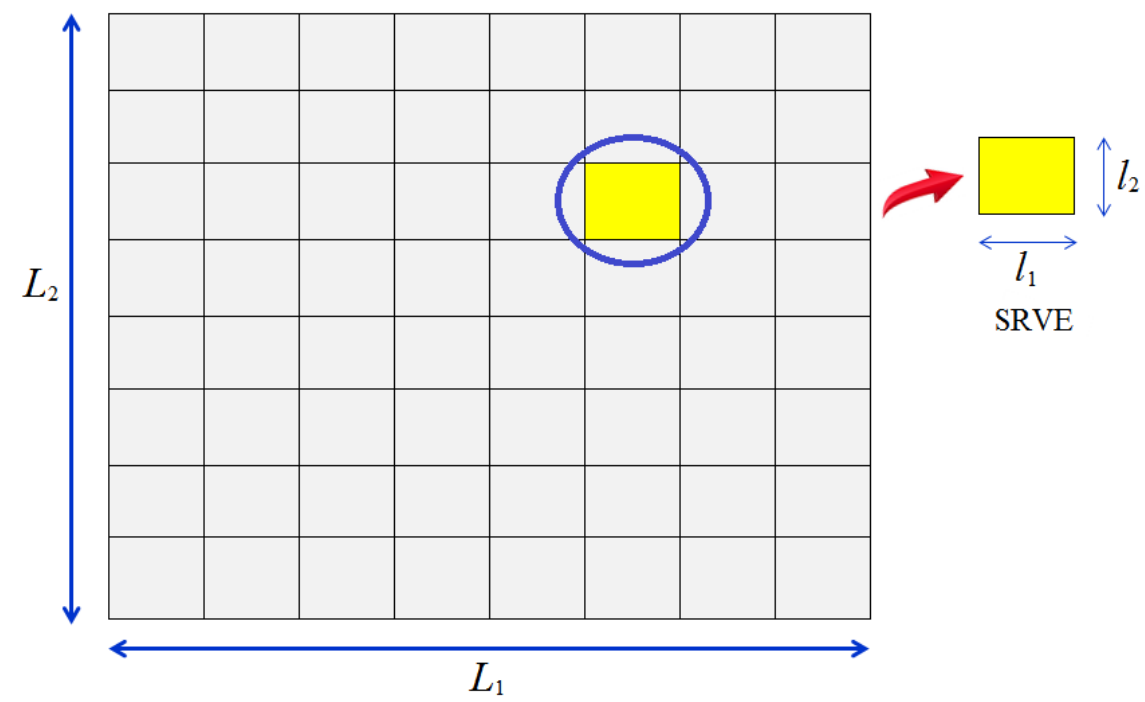

Fig. 5 SRVE based approach for analyzing spatially random two dimensional systems

mechanical properties of the entire irregular lattice can be computed by assembling the RUCEs based on equilibrium and compatibility conditions. The concept of SRVE for analyzing one dimensional beam-like structures with random material properties and crack density is first adopted by Naskar et al. (2017). In this paper, we have generalized the concept for stochastic analysis of two-dimensional plate-like structures with randomly inhomogeneous form of uncertainty (Mukhopadhyay and Adhikari (2017b)). It can be noted that spatially correlated material properties can be conveniently accounted in this approach based on the Karhunen-Loève expansion. The adoption of SRVEs in a plate-like structure is shown in the figure 5, wherein the two-dimensional space is divided into a finite number of stochastic elements (SRVEs) having dimensions of $l_{1}$ and $l_{2}$ in two mutually perpendicular directions of the two-dimensional structure. Each of the SRVEs possesses different material and structural properties. Here, a parameter characteristic length $(r)$ can be defined as: $r=\frac{l_{1}}{L_{1}}=\frac{l_{2}}{L_{2}}=\frac{1}{N_{d}}$, where $N_{d}$ denotes the number of divisions along the two dimensions of the plate. As per the proposed concept of SRVE, the size (/number) of SRVE is independent of the discretization in a finite element based numerical solution that could be adopted for dynamic/ stability analysis of the composite plate. The size (/number) of SRVE would normally be govorned by the spatial distribution of structural and material attributes along with the correlation length. Once the size of a SRVE is decided, they could be discretized following conventional finite element analysis using a mesh convergence study. In the present analysis, we have only considered spatial 
variation of material properties; but future studies could include random spatial variation of microstructural properties (such as micro-scale damage) using the proposed SRVE based framework. In such problems, appropriate finite element meshing schemes would need to be adopted for each of the SRVEs.

\subsection{Characterization of correlated material properties based on Karhunen-Loève expansion}

Random fields are useful for modelling variables which have correlated spatial randomness. When spatial variation of structural and material properties are considered in a randomly inhomogeneous structural system, the properties are often found to be spatially correlated. The conventional approach to deal with such random fields is to discretize it into different finite number of random variables. Available schemes for discretizing the random fields can be classified into three groups, point discretization (e.g., midpoint (Klintworth and Stronge (1988)), shape function (Liu et al. (1986a, b)), integration point method (Matthies et al. (1997)), optimal linear estimate (Li and Der (1993))); series expansion (e.g., orthogonal series expansion (Zhang and Ellingwood (1994))), and average discretization (e.g., spatial average (Vanmarcke (1983), Vanmarcke and Grigoriu (1983)), weighted integral (Deodatis (1991), Deodatis and Shinozuka (1991))).

The beneficial alternative for discretizing the random field is representing it in a generalized Fourier type of series such as Karhunen-Loève (KL) expansion (Karhunen (1947); Loève, (1977)). Let us consider a random field $\mathcal{H}(\boldsymbol{x}, \theta)$ with covariance function $\Gamma_{\mathcal{H}}\left(\boldsymbol{x}_{1}, \boldsymbol{x}_{2}\right)$ defined in the probability space $(\theta, F, P)$. The KL expansion for $\mathcal{H}(\boldsymbol{x}, \theta)$ can be expressed in the following form

$$
\mathcal{H}(\boldsymbol{x}, \theta)=\overline{\mathcal{H}}(\boldsymbol{x})+\sum_{i=1}^{\infty} \sqrt{\lambda_{i}} \xi_{i}(\theta) \psi_{i}(\boldsymbol{x})
$$

where $\left\{\xi_{i}(\theta)\right\}$ represents a set of random variables with no correlation. $\left\{\lambda_{i}\right\}$ and $\left\{\psi_{i}(x)\right\}$ denote the eigenvalues and eigenfunctions of the covariance kernel $\Gamma_{\mathcal{H}}\left(\boldsymbol{x}_{1}, \boldsymbol{x}_{2}\right)$, satisfying the integral equation

$$
\int_{\mathfrak{R}^{N}} \Gamma_{\mathcal{H}}\left(\boldsymbol{x}_{1}, \boldsymbol{x}_{2}\right) \psi_{i}\left(\boldsymbol{x}_{1}\right) d \boldsymbol{x}_{1}=\lambda_{i} \psi_{i}\left(\boldsymbol{x}_{2}\right)
$$

In practice, the infinite series in Equation (24) is truncated as 


$$
\tilde{\mathcal{H}}(\boldsymbol{x}, \theta) \cong \overline{\mathcal{H}}(\boldsymbol{x})+\sum_{i=1}^{N} \sqrt{\lambda_{i}} \xi_{i}(\theta) f_{i}(\boldsymbol{x})
$$

The above expression approaches to $\mathcal{H}(\boldsymbol{x}, \theta)$ in a mean square sense for $N \rightarrow \infty$. The finite element method (Huang et al. (2001)) can be applied to compute the eigensolutions for any covariance function and domain in the random field. In case of linear and exponential covariance functions along with a simple domain, the eigen solutions are feasible to be obtained analytically (Huang et al (2001)). Once $\Gamma_{\mathcal{H}}(s)$ and its eigen solutions are computed, the parameterization of $\tilde{\mathcal{H}}(\boldsymbol{x}, \theta)$ is carried out by the KL approximation of Gaussian image,

$$
\tilde{\mathcal{H}}(\boldsymbol{x}, \theta) \cong G\left[\overline{\mathcal{H}}(\boldsymbol{x})+\sum_{i=1}^{N} \sqrt{\lambda_{i}} \xi_{i}(\theta) f_{i}(\boldsymbol{x})\right]
$$

As per Equation (27), the KL approximation gives a parametric representation of $\tilde{\mathcal{H}}(\boldsymbol{x}, \theta)$ with $M$ random variables. It can be noted that this is not the only available method for discretizing the random field $\mathcal{H}(\boldsymbol{x}, \theta)$. However, KL expansion has uniqueness and error minimization properties that make it a superior choice over the other methods (Huang et al. (2001)).

In the present article, the stochastic material properties (micro/ macro-mechanical) are modelled as random fields and these are discretized using the KL expansion. To be specific, lognormal random fields are considered for modelling the correlated material properties. The covariance function is expressed as

$$
\Gamma_{\alpha z}=\sigma_{\alpha z}^{2} \mathrm{e}^{\left(-\left|y_{1}-y_{2}\right| / b_{y}\right)+\left(-\left|z_{1}-z_{2}\right| / b_{z}\right)}
$$

where $b_{y}$ and $b_{z}$ represent the two planar directions. These parameters control the rate of covariance decay. The eigensolutions of the covariance function can be obtained by solving the integral analytically (refer to Equation 25)

$$
\lambda_{i} \psi_{i}\left(y_{2}, z_{2}\right)=\int_{-a_{1}}^{a_{1}} \int_{-a_{2}}^{a_{2}} \Gamma\left(y_{1}, z_{1} ; y_{2}, z_{2}\right) \psi_{i}\left(y_{1}, z_{1}\right) d y_{1} d z_{1}
$$

where, $-a_{1} \leq y \leq a_{1}$ and $-a_{2} \leq z \leq a_{2}$. Assuming that the eigen-solution can be separated in $\mathrm{y}$ and $\mathrm{z}$ directions and substituting the covariance function 


$$
\begin{aligned}
& \psi_{i}\left(y_{2}, z_{2}\right)=\psi_{i}^{(y)}\left(y_{2}\right) \psi_{i}^{(z)}\left(z_{2}\right) \\
& \lambda_{i}\left(y_{2}, z_{2}\right)=\lambda_{i}^{(y)}\left(y_{2}\right) \lambda_{i}^{(z)}\left(z_{2}\right)
\end{aligned}
$$

, the solution of equation (29) can be reduced to the product of the solutions of two equations having the form

$$
\lambda_{i}^{(y)} \psi_{i}^{(y)}\left(y_{1}\right)=\int_{-a_{1}}^{a_{1}} e^{\left(-\left|y_{1}-y_{2}\right| / b_{y}\right)} \psi_{i}^{(y)}\left(y_{2}\right) d y_{2}
$$

Solution of the above equation, which is the eigensolution of an exponential covariance kernel with a onedimensional random field, can be obtained as

$$
\left\{\begin{array}{l}
\psi_{i}(\xi)=\frac{\cos \left(\omega_{i} \xi\right)}{\sqrt{a+\frac{\sin \left(2 \omega_{i} \xi\right)}{2 \omega_{i}}}} \lambda_{i}=\frac{2 \sigma_{\alpha_{z}}^{2} b}{\omega_{i}^{2}+b^{2}} \quad \text { for } i=\text { odd } \\
\psi_{i}(\xi)=\frac{\sin \left(\omega_{i}^{*} \xi\right)}{\sqrt{a-\frac{\sin \left(2 \omega_{i}^{*} \xi\right)}{2 \omega_{i}^{*}}}} \lambda_{i}^{*}=\frac{2 \sigma_{\alpha_{z}}^{2} b}{\omega_{i}^{* 2}+b^{2}} \quad \text { for } i=\text { even }
\end{array}\right.
$$

Where $b=1 / b_{y}$ or $1 / b_{z}$ and $a=a_{1}$ or $a_{2}$. The parameter $\xi$ is either y or z. Here $\omega_{i}$ and $\omega_{i}^{*}$ are computed from the solutions of the following equations $b-\omega_{i} \tan \left(\omega_{i} a\right)=0$ and $\omega_{i}+b \tan \left(\omega_{i} a\right)=0$, respectively. It can be noted that the KL expansion was formulated for discretization following gaussian random fields. In case of lognormal random fields, as considered in the present study, the KL expansion is formulated on its gaussian image. In the current study of laminated composite plates, the spatially correlated properties are parameterized by the respective mean values (considered to be same as the deterministic value of a particular parameter), the coefficient of variation $(\mathrm{COV})$ and two correlation parameters. The degree of stochasticity is defined as the coefficient of variation $(\Delta)$ of a particular stochastic input parameter. 


\subsection{Description of the uncertainty quantification framework}

\subsubsection{Monte Carlo simulation}

Uncertainty quantification is part of modern structural analysis problems. Practical structural systems need to face uncertainty, variability and ambiguity on a constant basis. Even after having unprecedented access to the information due to recent improvement in various technologies, it is impossible to accurately predict future structural behaviour during its service life. Monte Carlo simulation, a computerized mathematical technique, lets us realize all the possible outcomes of a structural system leading to better and robust designs for the intended performances. This technique was first used by the engineers and scientists developing the atom bomb and it was named after a Monaco resort town Monte Carlo. Since the introduction during World War II, this technique has been applied to model various physical and conceptual systems across different fields covering engineering, manufacturing, energy, finance, insurance, project management, transportation and environment.

Monte Carlo simulation (MCS) furnishes a range of prospective outcomes along with their respective probability of occurrence. This technique performs uncertainty quantification by forming probabilistic simulations of all prospective results accounting a wide range of values from the probability distributions of any factor having an inherent uncertainty. This method simulates the outputs multiple times, using a different set of random values each time, drawn from the probability distribution of stochastic input parameters. Depending upon the nature of stochasticity, a Monte Carlo simulation may involve thousands or tens of thousands of realizations (/function evaluations) before it can provide a converged result depicting the distributions of possible outcome values of the response quantities of interest. Thus Monte Carlo simulation provides not only a comprehensive idea of what could happen, but also how likely it is to happen i.e. the probability of occurrence.

The mean (/expected) value of a function $\bar{f}(x)$ having an $n$ dimensional vector of random variables and a joint probability density function $\bar{\phi}(x)$, is expressed as

$$
\mu_{\bar{f}}=E[\bar{f}(x)]=\int_{\Omega} \bar{f}(x) \bar{\phi}(x) d x
$$


The variance of the function $\bar{f}(x)$ is given as,

$$
\sigma_{\bar{f}}^{2}=\operatorname{Var}[\bar{f}(x)]=\int_{\Omega}\left(\bar{f}(x)-\mu_{\bar{f}}\right)^{2} \bar{\phi}(x) d x
$$

The multidimensional integrals, as shown in equation (34) and (35) are difficult to compute analytically various types of joint density functions. Moreover, the integrand function $\bar{f}(x)$ may not always be available in an analytical form for the problem under consideration. Thus the only alternative way is to calculate it by numerical means. It can be computed using the MCS approach, wherein $N$ number of sample points is generated following a suitable sampling scheme in the random variable space of $n$ dimensions. The $N$ number of samples drawn from a dataset must follow the same distribution as $\bar{\varphi}(x)$. The function $\bar{f}(x)$ is computed at each of the sampling points $x_{i}$ of the sample set $\chi=\left\{x_{1}, \ldots \ldots \ldots \ldots, x_{N}\right\}$. Thus, the integral for the expected value can be expressed in the form of averaging operator as

$$
\mu_{\bar{f}}=E[\bar{f}(x)]=\frac{1}{N} \sum_{i=1}^{N} \bar{f}\left(x_{i}\right)
$$

In a similar manner, using sampled values of MCS as above, the equation (35) leads to

$$
\sigma_{\bar{f}}^{2}=\operatorname{Var}[\bar{f}(x)]=\frac{1}{N-1} \sum_{i=1}^{N}\left(\bar{f}\left(x_{i}\right)-\mu_{\bar{f}}\right)^{2}
$$

Thus the statistical moments can be obtained using a brute force MCS based approach, which is often computationally very intensive due to the evaluation of function $\bar{f}\left(x_{i}\right)$ corresponding to the $N$-sampling points $x_{i}$, where $N \sim 10^{4}$. The noteworthy fact in this context is the adoption of a surrogate based Monte Carlo simulation approach in the present study that reduces the computational burden of traditional (i.e. brute force) Monte Carlo simulation to a significant extent.

\subsubsection{Modelling of source-uncertainty at the input level}

The stochasticity in material properties (micro/ macro- mechanical properties) and geometric properties (like ply-orientation angle and thickness of plate) are considered as stochastic input parameters for analyzing the probabilistic dynamic and buckling characteristics of laminated composite plates. In the 
present article, two separate forms of analysis have been performed considering the stochasticity in micromechanical and macro-mechanical properties to understand and ascertain the cascading effect in uncertainty propagation on a comparative basis. For analysing the effect of various source-uncertainties, the following four cases of stochasticity are considered

(i) Compound effect for the simultaneous variation of macro-mechanical material properties (such as Young's moduli, shear moduli, mass density and Poisson's ratio) and geometric properties (such as plyorientation angle and thickness of laminae)

$$
\begin{aligned}
g_{\text {macro }}^{C}\{ & \left.E_{1}(\bar{\omega}), E_{2}(\bar{\omega}), G_{12}(\bar{\omega}), G_{13}(\bar{\omega}), G_{23}(\bar{\omega}), \mu(\bar{\omega}), \rho(\bar{\omega}), \theta(\bar{\omega}), t(\bar{\omega})\right\} \\
= & \left\{\begin{array}{l}
\phi_{\text {macro }}^{1}\left(E_{1(1,1)} \ldots . E_{1(e, l)}\right), \phi_{\text {macro }}^{2}\left(E_{2(1,1)} \ldots . E_{2(e, l)}\right), \phi_{\text {macro }}^{3}\left(G_{12(1,1)} \ldots G_{12(e, l)}\right), \\
\phi_{\text {macro }}^{4}\left(G_{13(1,1)} \ldots G_{13(e, l)}\right), \phi_{\text {macro }}^{5}\left(G_{23(1,1)} \ldots . G_{23(e, l)}\right), \phi_{\text {macro }}^{6}\left(\mu_{(1,1)} \ldots \mu_{(e, l)}\right), \\
\phi_{\text {macro }}^{7}\left(\rho_{(1,1)} \ldots \rho_{(e, l)}\right), \phi_{\text {macro }}^{8}\left(\theta_{(1,1)} \ldots . \theta_{(e, l)}\right), \phi_{\text {macro }}^{9}\left(t_{(1,1)} \ldots t_{(e, l)}\right)
\end{array}\right\}
\end{aligned}
$$

(ii) Compound effect for the simultaneous variation of micro-mechanical material properties such as Young's moduli of fibre and matrix, shear moduli of fibre and matrix, Poisson ratios of fibre and matrix, mass densities of fibre and matrix and volume fraction along with geometric properties (ply orientation angle and thickness of laminae)

$$
\begin{aligned}
g_{\text {micro }}^{C} & \left\{E_{1 f}(\bar{\omega}), E_{2 f}(\bar{\omega}), E_{m}(\bar{\omega}), G_{f}(\bar{\omega}), G_{m}(\bar{\omega}), \mu_{f}(\bar{\omega}), \mu_{m}(\bar{\omega}), \rho_{f}(\bar{\omega}), \rho_{m}(\bar{\omega}), V_{f}(\bar{\omega}), \theta(\bar{\omega}), t(\bar{\omega})\right\} \\
= & \left\{\begin{array}{l}
\phi_{\text {micro }}^{1}\left(E_{1 f(1,1)} \ldots E_{1 f(e, l)}\right), \phi_{\text {micro }}^{2}\left(E_{2 f(1,1)} \ldots E_{2 f(e, l)}\right), \phi_{\text {micro }}^{3}\left(E_{m(1,1)} \ldots . E_{m(e, l)}\right), \phi_{\text {micro }}^{4}\left(G_{f(1,1)} \ldots . G_{f(e, l)}\right), \\
\phi_{\text {micro }}^{5}\left(G_{m(1,1)} \ldots G_{m(e, l)}\right), \phi_{\text {micro }}^{6}\left(\mu_{f(1,1)} \ldots, \mu_{f(e, l)}\right), \phi_{\text {micro }}^{7}\left(\mu_{m(1,1)} \ldots, \mu_{m(e, l)}\right), \phi_{\text {micro }}^{8}\left(\rho_{f(1,1)} \ldots \rho_{f(e, l)}\right), \\
\phi_{\text {micro }}^{9}\left(\rho_{m(1,1)} \ldots \rho_{m(e, l)}\right), \phi_{\text {micro }}^{10}\left(V_{f(1,1)} \ldots . V_{f(e, l)}\right), \phi_{\text {micro }}^{11}\left(\theta_{f(1,1)} \ldots \theta_{f(e, l)}\right), \phi_{\text {micro }}^{12}\left(t_{f(1,1)} \ldots . t_{f(e, l)}\right)
\end{array}\right\}
\end{aligned}
$$

(iii) Individual effect for the variation of a single macro-mechanical property

$$
g_{\text {macro }}^{I}\left\{\kappa_{M}(\bar{\omega})\right\}=\left\{\phi_{\text {macro }}^{1}\left(\kappa_{M(1,1)} \ldots \kappa_{M(e, l)}\right)\right\}
$$

(iv) Individual effect for the variation of a single micro-mechanical property

$$
g_{\text {micro }}^{I}\left\{\kappa_{m}(\bar{\omega})\right\}=\left\{\phi_{\text {micro }}^{1}\left(\kappa_{m(1,1)} \ldots \kappa_{m(e, l)}\right)\right\}
$$

Here $\phi$ is a symbolic operator that generates a set of input parameters for carrying out the Monte Carlo simulation. The parameters $E_{1(i, j)}, E_{2(i, j)}, G_{12(i, j)}, G_{13(i, j)}, G_{23(i, j)}, \mu_{(i, j)}, \rho_{(i, j)}, \theta_{(i, j)}, t_{(i, j)}$ denote the longitudinal Young's modulus, transverse Young's modulus, shear moduli, Possoin's ratio, mass density, ply 
orientation angle and thickness of lamina respectively (with conventional notations) for the $i^{\text {th }}$ SRVE situated in the $j^{\text {th }}$ layer, where $i=1,2,3, \ldots, e$ and $j=1,2,3, \ldots, l$. For the stochasticity in micromechanical properties, $E_{1 f(i, j)}, E_{2 f(i, j)}, E_{m(i, j)}, G_{f(i, j)}, G_{m(i, j)}, \mu_{f(i, j)}, \mu_{m(i, j)}, \rho_{f(i, j)}, \rho_{m(i, j)}, V_{f(i, j)}, \theta_{(i, j)}, t_{(i, j)}$ denote (following conventional notations) Young's moduli of fibre in longitudinal and transverse directions, Young's modulus of matrix, shear modulus of fibre and matrix, Poisson's ratio of fibre and matrix, mass density of fibre and matrix and volume fraction, ply orientation angle and thickness respectively corresponding to $i^{\text {th }}$ layer, respectively for the $i^{\text {th }}$ SRVE situated in the $j^{\text {th }}$ layer, where $i=1,2$, $3, \ldots, e$ and $j=1,2,3, \ldots, l$. The quantities $\kappa_{M}$ and $\kappa_{m}$ denote any one of the macromechanical and micromechanical properties. The material properties are considered to vary spatially (correlated variation following the KL expansion) for both the macromechanical and micromechanical analyses. However, considering practical aspects of modelling uncertainty in the geometric parameters, spatial variation is not considered for ply orientation angle and thickness of lamina; rather a layer-wise uncorrelated random variation is considered for the two geometric parameters (i.e. $\theta_{(1, j)}=\theta_{(2, j)}=\theta_{(3, j)}=\ldots=\theta_{(e, j)}$ and $t_{(1, j)}=t_{(2, j)}=t_{(3, j)}=\ldots=t_{(e, j)}$, for the $j^{\text {th }}$ lamina $)$.

\subsubsection{Propagation of uncertainty based on HDMR coupled with DMORPH algorithm}

In a typical problem of uncertainty analysis, there are normally three aspects that need to be dealt with. The first aspect is source-uncertainty modelling at the input level, which is discussed in the preceding subsection. After the uncertainty in material and structural attributes is modelled in a practically relevant way, the next concern is propagating the effect of uncertainty from the local inputlevel to the global level of quantifying output responses following a bottom-up framework. As discussed in section 1, direct Monte Carlo simulation being a computationally intensive approach, we have adopted a surrogate based uncertainty propagation scheme for the present analysis. To achieve computational efficiency, a HDMR based surrogate modelling framework coupled with the DMORPH algorithm is developed in conjunction with the probabilistic finite element model of composite plates as presented in figure 6. 


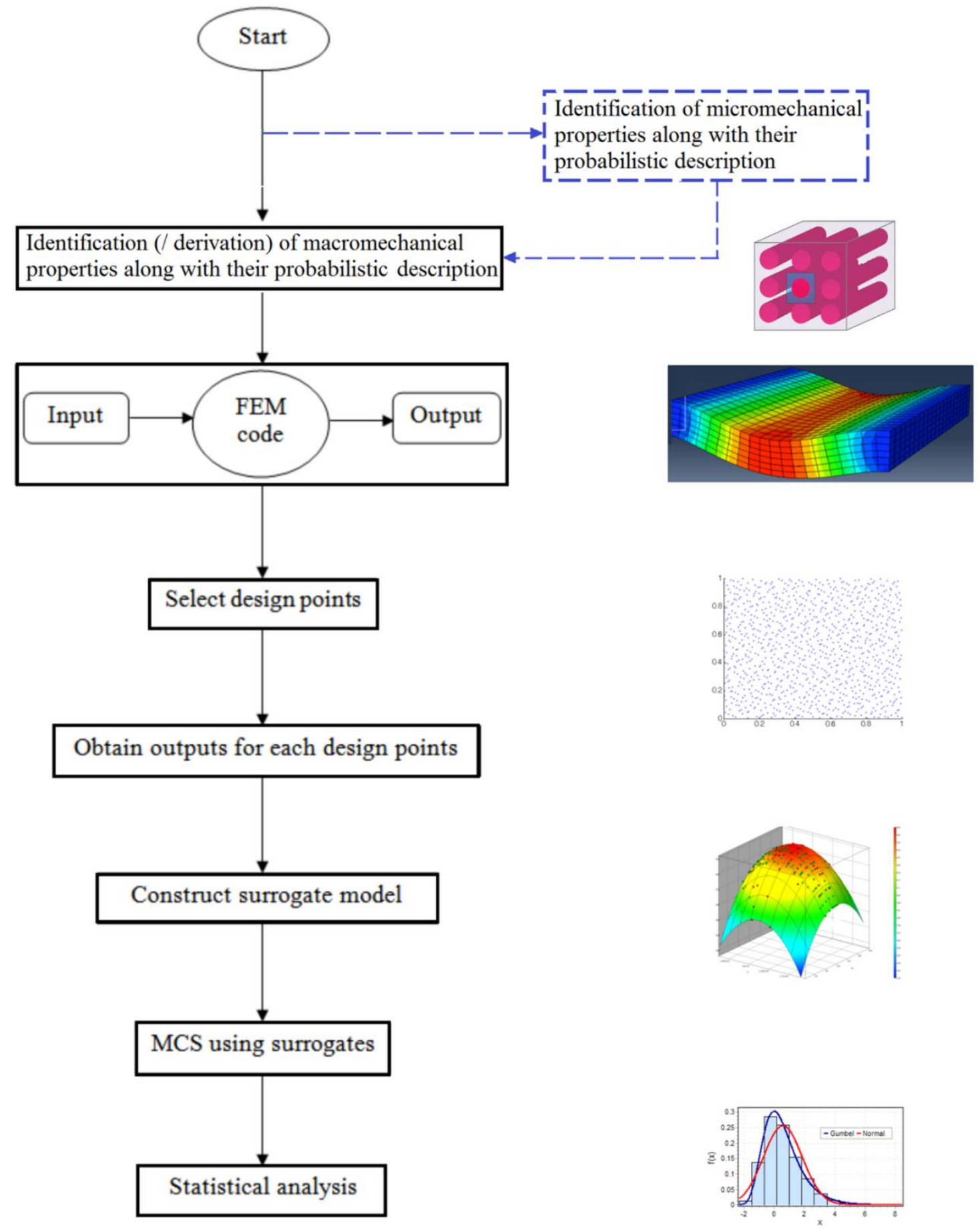

Fig. 6 Flowchart for stochastic macro and micro mechanical analysis of laminated composites based on surrogate models (Representative figures of finite element analysis, sobol's quasi-random sampling, surrogate modelling and uncertainty quantification are shown corresponding to the respective steps)

The surrogate models are constructed by choosing the sample points optimally from a domain $R^{n}$. All input variables are rescaled in the range of $0 \leq x_{i} \leq 1$, where $x_{i}$ denotes the $i^{\text {th }}$ input parameter. Generation of the random sample points is an important aspect to form the HDMR model because the quality of the random sample points governs the convergence rate and prediction accuracy. Quasi-random 
sequences (Niederreiter (1992)) (such as Halton (Halton (1960)), Sobol' (Sobol (1967)), Faure (Faure (1992))) having low discrepancy are often used for generating random sample points that ensures a uniform distribution of input sample points in the design domain. It essentially results in a faster convergence rate compared to pseudo-random sample points. In this work, Sobol' sequence is used to generate the input sample set as it shows a better convergence rate than Faure and Halton sequences (Galanti and Jung (1997)). It can be noted in this context that in a surrogate based approach, first the surrogate model is constructed using few optimally chosen design points. The same number (number of design points) of finite element simulations (Ifunction evaluations) is required to be performed for the surrogate model formation. Here the HDMR model replaces the original finite element model (expensive) effectively by an efficient mathematical/statistical model. After the HDMR based surrogate model is constructed, thousands of virtual simulations can be conducted for various random combinations of the input parameters using the efficient HDMR model.

\section{Results and discussion}

In this article, numerical results for stochastic dynamic and stability analyses are presented for a three layered graphite-epoxy angle-ply ([45\% $\left.\left./ 45^{\circ} / 45^{\circ}\right]\right)$ composite square plate, unless otherwise mentioned. A practically relavent randomly inhomogeneous (Mukhopadhyay and Adhikari (2017b)) model of stochasticity with spatially correlated system parameters are considered for characterizing the first three modes of vibration and buckling of composite plates. Results are presented for two distinct cases: stochasticity in micromechanical and macromechanical material properties (refer to equation 38 41). The deterministic micromechanical properties (E-glass 21xK43Gevetex/ 3501-6epoxy) of composite material are shown in Table 1 (Soden et al. (1998)). Applying Halpin-Tsai principle (Jones (1999)) the deterministic macromechanical material properties are obtained with a volume fraction $\left(V_{f}\right)$ of 0.61 (refer to Table 2). Thus, for the case of stochasticity in micromechanical properties, the material atributes presented in Table 1 are assumed as the source of stochasticity along with the uncertain geometric parameters $\left(g_{\text {micro }}^{C}\right)$ and thereby the macromechanical material properties are obtained based on Halpin- Tsai principle to perform further analysis for quantifying uncertainty. For the case of stochasticity 
Table 1 Deterministic micromechanical material properties of composites

\begin{tabular}{c|c}
\hline Property & Value \\
\hline $\begin{array}{c}\text { Longitudinal Young's modulus of fibre }\left(E_{1 f}\right) \\
\text { Transverse Young's modulus of fibre }\left(E_{2 f}\right)\end{array}$ & $80 \mathrm{GPa}$ \\
Poisson's ratio of fibre $\left(\mu_{f}\right)$ & $80 \mathrm{GPa}$ \\
Shear modulus of matrix $\left(G_{f}\right)$ & 0.2 \\
Mass density of fibre $\left(\rho_{f}\right)$ & $33.33 \mathrm{GPa}$ \\
Mass density of matrix $\left(\rho_{m}\right)$ & $2.55 \mathrm{gm} / \mathrm{cc}$ \\
Young's modulus of matrix $\left(E_{m}\right)$ & $1.265 \mathrm{gm} / \mathrm{cc}$ \\
Shear modulus of matrix $\left(G_{m}\right)$ & $4.2 \mathrm{GPa}$ \\
Poisson's ratio of matrix $\left(\mu_{m}\right)$ & $1.567 \mathrm{GPa}$ \\
Fibre volume fraction $\left(V_{f}\right)$ & 0.34 \\
\hline
\end{tabular}

Table 2 Deterministic macro-mechanical material properties of composites $\left(V_{f}=0.61\right)$

\begin{tabular}{c|c}
\hline Property & Value \\
\hline Longitudinal Young's modulus $\left(E_{1}\right)$ & $50.438 \mathrm{GPa}$ \\
Transverse Young's modulus $\left(E_{2}\right)$ & $9.952 \mathrm{GPa}$ \\
Poisson's ratio $\left(\mu_{12}\right)$ & 0.2546 \\
In-plane shear modulus $\left(G_{12}\right)$ & $3.742 \mathrm{GPa}$ \\
Mass density $(\rho)$ & $2.049 \mathrm{gm} / \mathrm{cc}$ \\
Shear modulus $\left(G_{13}\right)$ & $3.742 \mathrm{GPa}$ \\
Transverse shear modulus $\left(G_{23}\right)$ & $2.094 \mathrm{GPa}$ \\
\hline
\end{tabular}


in macromechanical material properties, the analysis commences one step ahead in the hierarchy i.e. the source-uncertainty is assumed in the macromechanical properties (as shown in Table 2) along with uncertain geometric parameters $\left(g_{\text {macro }}^{C}\right)$. Subsequently, the results obtained from these two different types of analyses are compared to ascertain the cascading effect in stochasticity. Non-dimensional results are presented following the scheme mentioned in the caption of Table 3 and 4.

\subsection{Stochastic dynamic analysis}

\subsubsection{Validation and convergence study}

In the surrogate assisted stochastic analysis of laminated composites, two different forms of validation and convergence study are needed to be carried out. The first validation is for the finite element model of composite plate along with mesh convergence study. A second type of validation is also needed here concerning the performance (efficiency and accuracy) of the surrogate model in predicting the responses along with a convergence study for minimizing the number of design points required for forming surrogate models.

The results for validation and convergence study of the finite element code of a composite plate are shown in Table 3, wherein non-dimensional natural frequencies are validated with the results available in scientific articles (Liew and Huang (2003)). Based on the results presented in Table 3, a mesh size of $9 \times 9$ is adopted for the finite element analysis hereafter. The optimum number of samples (drawn from Sobol sequence) to construct the surrogate models are decided based on the comparative performance (four different statistical parameters: minimum value, maximum value, mean value and standard deviation) with respect to direct Monte Carlo simulation. The convergence results for macro and micro mechanical analyses showing the values of absolute error with different sample size are presented in figure 7 and 8 . From the figures it is evident that a sample size of 1024 provides reasonably accurate results for the natural frequencies. To further examine the prediction capabilities of the surrogate models, scatter plots are presented for the macro and micro mechanical analyses in figures $9(\mathrm{a}-\mathrm{c})$ and $10(\mathrm{a}-\mathrm{c})$, respectively. Negligible deviation of the sample points from the diagonal line corresponding to the sample size of 1024 indicates the accuracy of prediction. The comparative probability density function plots on the basis of 
Table 3 The convergence study of frequency parameters $\left[\bar{\omega}=\left(\omega b^{2} / \pi^{2}\right) \sqrt{\left(\rho h / D_{0}\right)}\right.$, where $\left.D_{0}=E_{2} h^{3} / 12\left(1-v_{12} v_{21}\right)\right]$ for cross-ply $\left(0^{\circ} / 90^{\circ} / 0^{\circ}\right)$ simply supported (SSSS) rectangular laminates

\begin{tabular}{|c|c|c|c|c|c|c|c|}
\hline \multirow[b]{2}{*}{$t / b$} & \multirow[b]{2}{*}{ Mesh } & \multicolumn{2}{|c|}{ Mode 1} & \multicolumn{2}{|c|}{ Mode 2} & \multicolumn{2}{|c|}{ Mode 3} \\
\hline & & $\begin{array}{c}\text { Present } \\
\text { FEM }\end{array}$ & Liew (1996) & $\begin{array}{l}\text { Present } \\
\text { FEM }\end{array}$ & Liew (1996) & $\begin{array}{c}\text { Present } \\
\text { FEM }\end{array}$ & Liew (1996) \\
\hline \multirow{6}{*}{0.001} & $5 \times 5$ & 6.8817 & \multirow{6}{*}{6.6253} & 9.7192 & \multirow{6}{*}{9.4653} & 24.4234 & \multirow{6}{*}{22.4607} \\
\hline & $6 \times 6$ & 6.7864 & & 9.6212 & & 24.0928 & \\
\hline & $7 \times 7$ & 6.7056 & & 9.5312 & & 23.4168 & \\
\hline & $8 \times 8$ & 6.6258 & & 9.4684 & & 22.4802 & \\
\hline & $9 \times 9$ & 6.6251 & & 9.4644 & & 22.4520 & \\
\hline & $10 \times 10$ & 6.6210 & & 9.4401 & & 22.2811 & \\
\hline \multirow{6}{*}{0.20} & $5 \times 5$ & 3.6867 & \multirow{6}{*}{3.5940} & 6.1626 & \multirow{6}{*}{5.7784} & 8.1347 & \multirow{6}{*}{7.3972} \\
\hline & $6 \times 6$ & 3.6314 & & 6.0189 & & 7.9167 & \\
\hline & $7 \times 7$ & 3.6145 & & 5.9180 & & 7.6512 & \\
\hline & $8 \times 8$ & 3.5978 & & 5.7337 & & 7.3368 & \\
\hline & $9 \times 9$ & 3.5913 & & 5.7331 & & 7.3345 & \\
\hline & $10 \times 10$ & 3.5839 & & 5.7036 & & 7.2366 & \\
\hline
\end{tabular}

surrogate based MCS corresponding to the sample size of 1024 and direct MCS are presented for the macro and micro mechanical analyses in figures $9(\mathrm{~d}-\mathrm{f})$ and $10(\mathrm{~d}-\mathrm{f})$, respectively. A good agreement between the probabilistic descriptions of natural frequencies corroborates the accurate prediction capability of the surrogate models for further analyses. It can be noted in this context that computational time required is exorbitently high for evaluating the probabilistic responses through full scale MCS because of the involvement of large number of finite element simulations ( 104). However, in case of the present surroagate based method, although a same sample size as the direct MCS is considered, the requirement of carrying out actual finite element simulations is much lesser compared to the direct MCS approach. Here it is equal to the number of samples required to form the HDMR based surrogate model (i.e. 1024). Hence, the computational intensiveness (time and effort) in terms of finite element analyses 


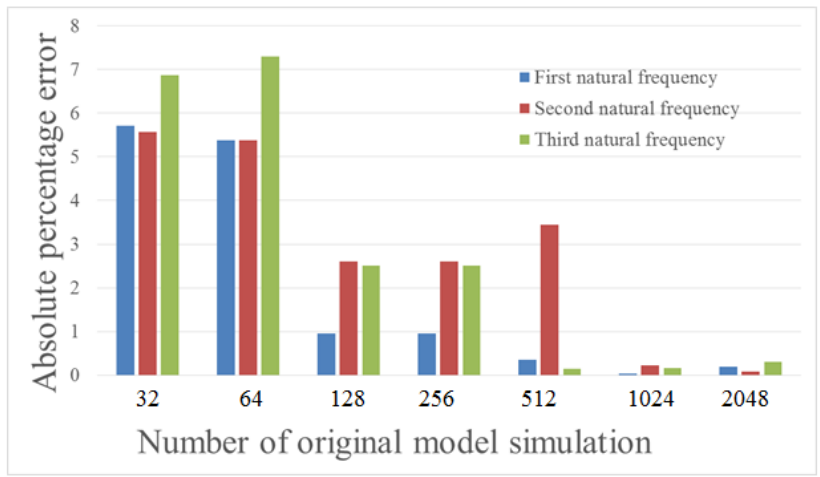

(a) Maximum

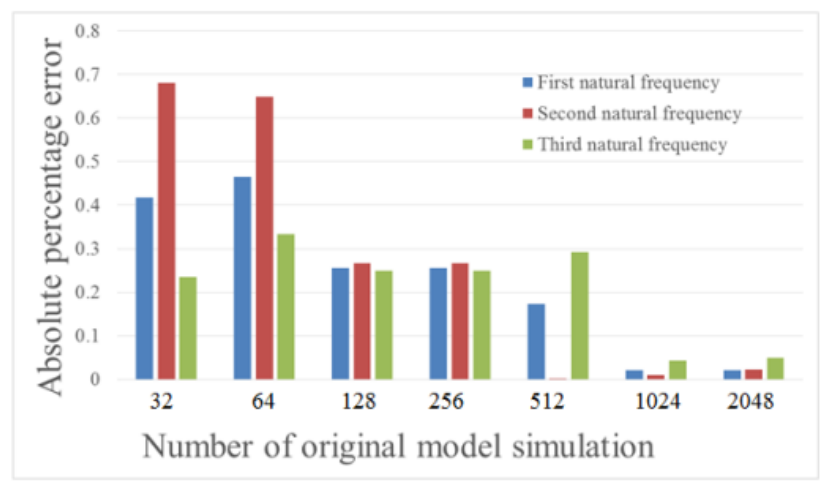

(c) Mean

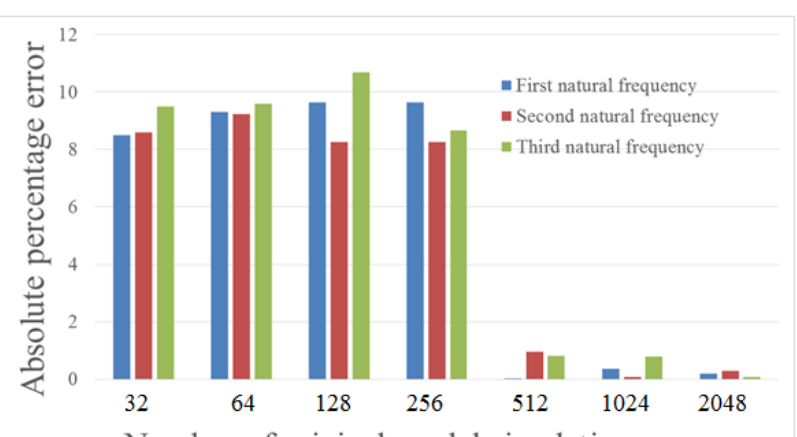

Number of original model simulation

(b) Minimum

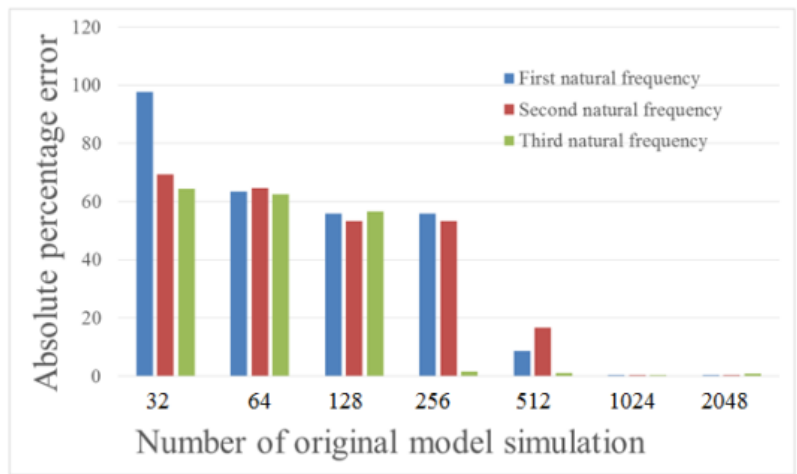

(d) Standard Deviation

Fig. 7 Absolute percentage error in minimum value, maximum value, mean value and standard deviation with respect to direct Monte Carlo simulation for macromechanical material properties for a coefficient of variation of 0.6 in the stochastic input parameters $\left(g_{\text {macro }}^{C}\right)$.

are decreased significantly in comparison to full-scale direct MCS.

\subsubsection{Results for stochastic dynamic analysis}

Having the FE model and the surrogate model validated, as shown in the preceding subsection, stochastic results are presented in this subsection for the first three modes of vibration for a composite plate with correlated spatially varying material properies $\left(g_{\text {macro }}^{C}\right.$ and $\left.g_{\text {micro }}^{C}\right)$. Figure 11 shows the probabilistic descriptions for first three natural frequencies considering different degrees of stochasticity for macro-mechanical and micro-mechanical material properties. For both the micro and macro mechanical analyses, response bounds are noticed to substantially increase with the increasing degree of stochasticity along with a marginal change in mean values. The probabilistic descriptions of the natural frequencies differ from each other on the basis of the adopted type of analysis. A micromechanical analysis, which is more accurate for considering the source uncertainty at a more elementary level, shows higher degree of variability in the global responses due to the cascading effect in stochasticity. 


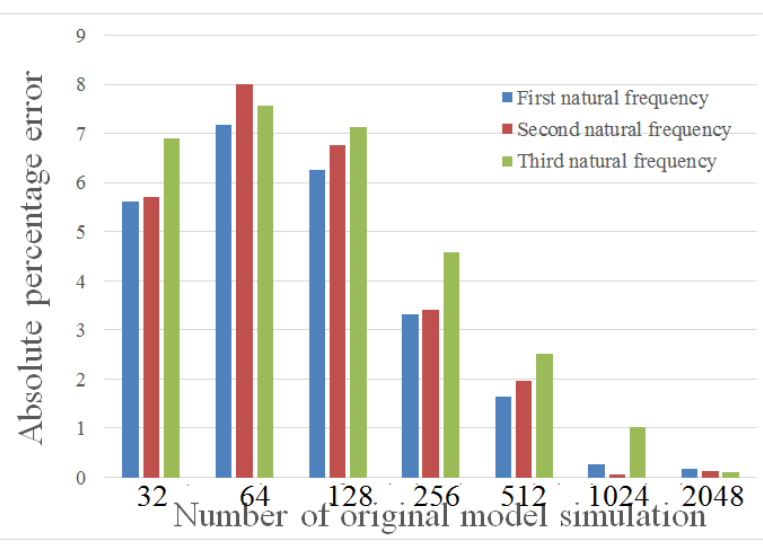

(a) Maximum

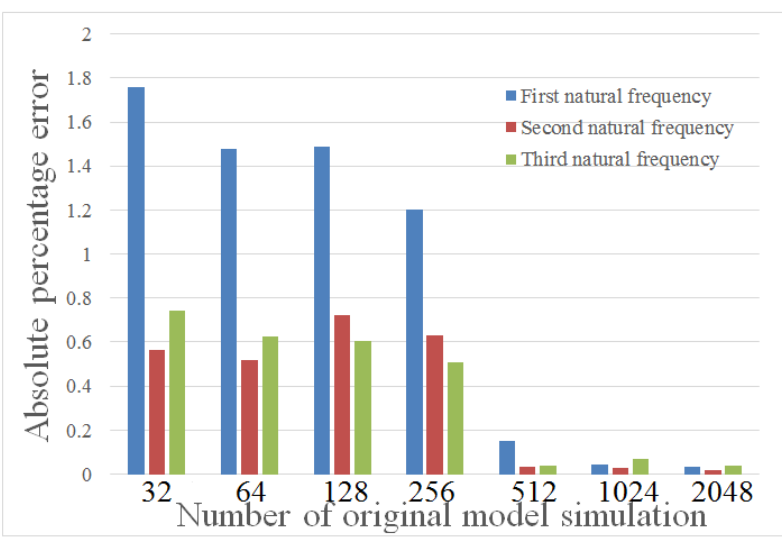

(c) Mean

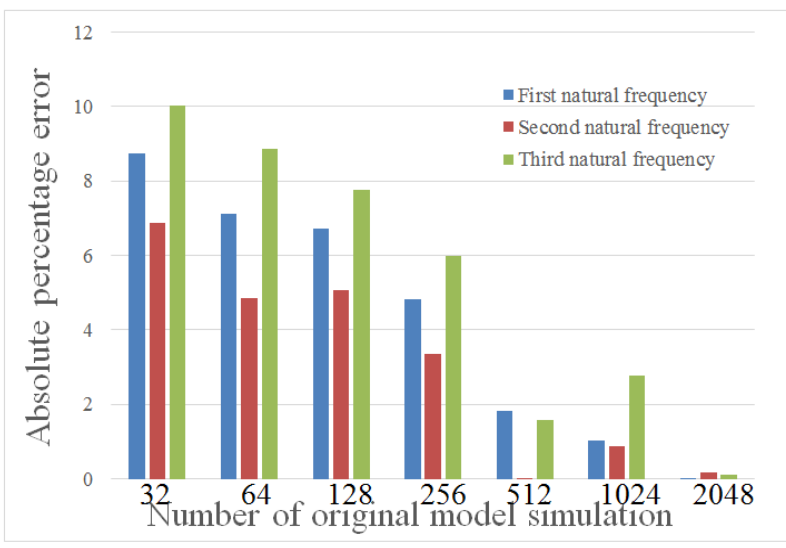

(b) Minimum

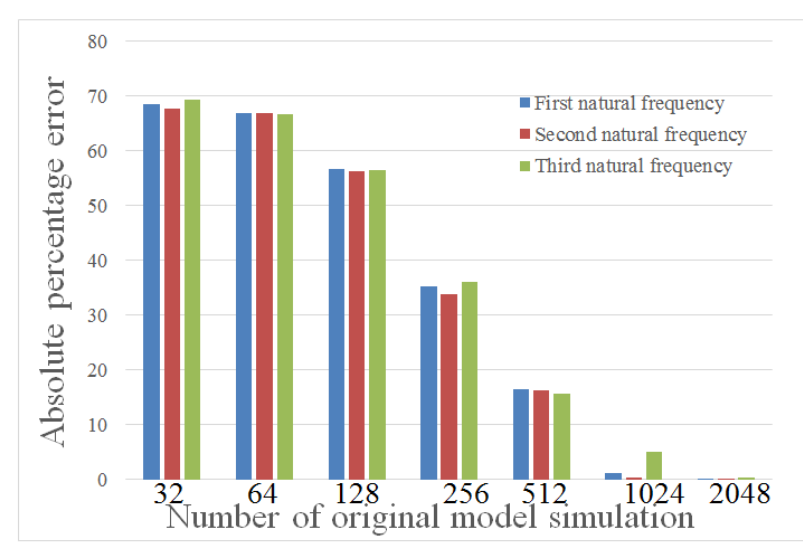

(d) Standard Deviation

Fig. 8 Absolute percentage error in maximum, minimum, mean and standard deviation with respect to original MCS considering a coefficient of variation of 0.6 in the stochastic input parameters $\left(g_{\text {micro }}^{C}\right)$.

The probabilistic variations for first three natural frequencies are investigated for various laminate configurations considering the $[\theta /-\theta / \theta]$ family of composites for stochasticity in micro-mechanical properties. The probabilistic descriptions presented in figure 12 show that the mean values, depending on the effective stiffness of the structure, decrease up to a critical value of $\theta=45^{\circ}$, and after this point they increase again for higher values of $\theta$. Figure 13 shows the influence of different boundary conditions on the stochastic natural frequencies of composite plates. Results are presented for simply supported (SSSS) and fixed (CCCC) boundaries at all the four edges considering stochasticity in micro-mechanical properties. The range of natural frequencies is found to vary depending on the stiffness of the system, following a similar trend as deterministic analysis. Figure 14 shows the effect of aspect ratio of the composite laminated plates on the probabilistic variation of natural frequencies considering stochasticity in micro-mechanical material properties. The stochastic natural frequencies are noticed to reduce with the increase in aspect ratio. 


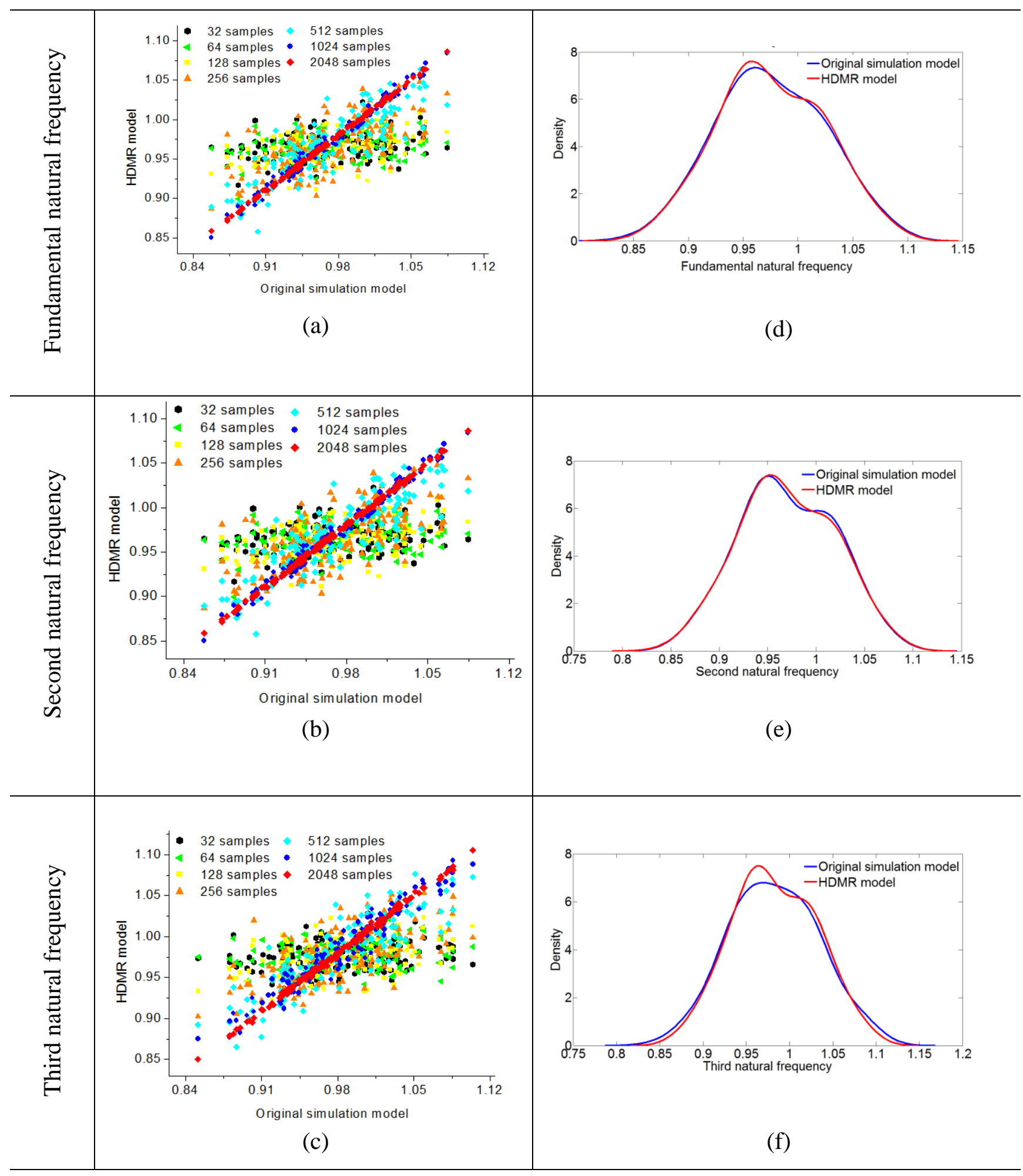

Fig. 9 (a-c) Scatter plots for HDMR based analysis (normalized on the basis of respective deterministic values) for various sample sizes of Sobol sequence considering the stochasticity in macro-mechanical attributes $\left(g_{\text {macro }}^{C}\right.$ ) with respect to direct MCS (with coefficient of variation of 0.6 in the stochastic input parameters) ; (d-f) Probability density function (pdf) of natural frequencies (normalized with respect to the corresponding deterministic values) for macro-mechanical attributes $\left(g_{\text {macro }}^{C}\right.$ ) obtained by direct MCS and HDMR model based on a sample size of 1024 (with coefficient of variation of 0.6 in the stochastic input parameters) 


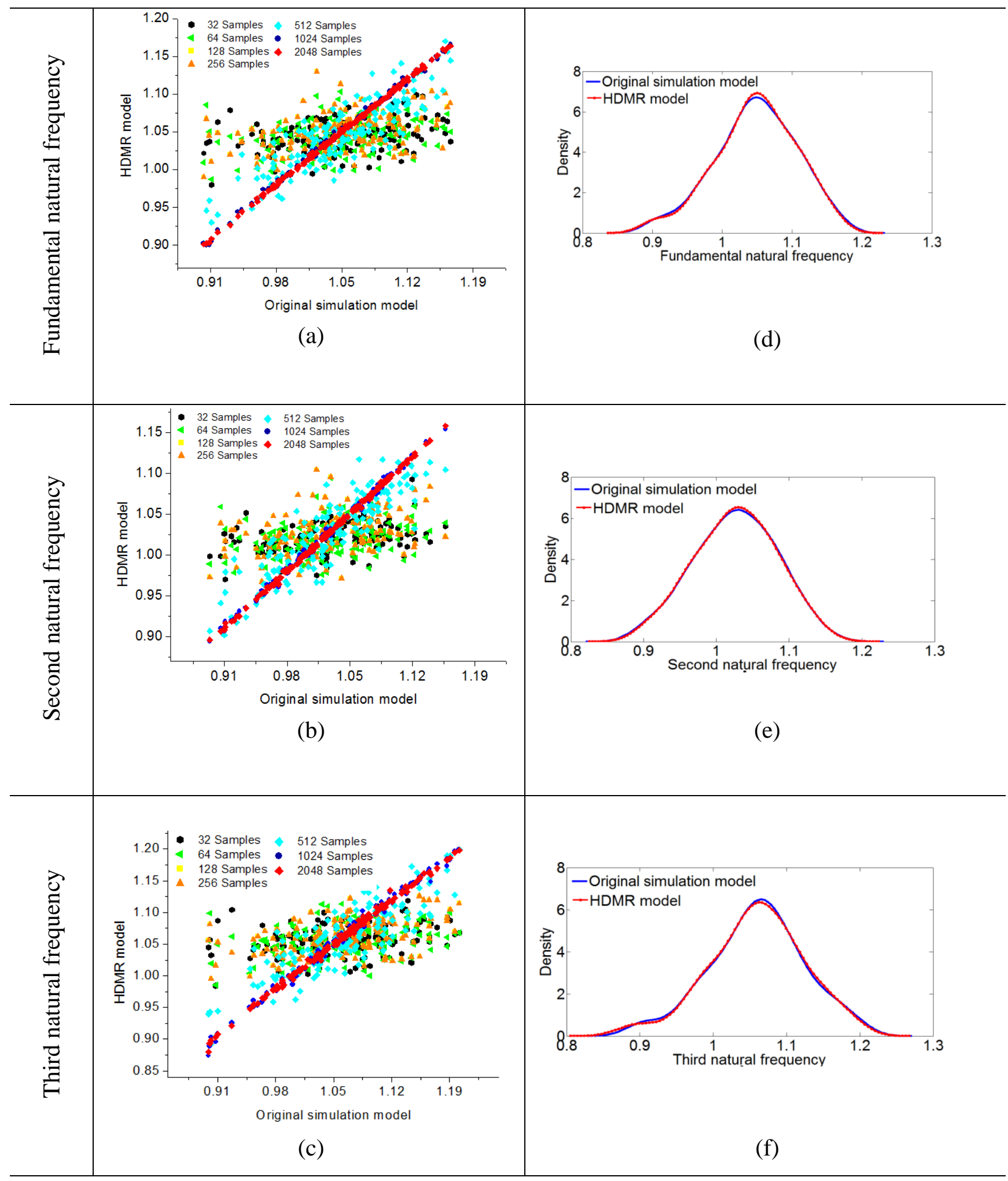

Fig. 10 (a-c) Scatter plots for HDMR based analysis (normalized on the basis of respective deterministic values) for various sample sizes of Sobol sequence considering the stochasticity in micro-mechanical attributes $\left(g_{\text {micro }}^{C}\right.$ ) with respect to direct MCS (with coefficient of variation of 0.6 in the stochastic input parameters) ; (d-f) Probability density function (pdf) of natural frequencies (normalized with respect to the corresponding deterministic values) for micro-mechanical attributes $\left(g_{\text {micro }}^{C}\right)$ obtained by direct MCS and HDMR model based on a sample size of 1024 (with coefficient of variation of 0.6 in the stochastic input parameters) 


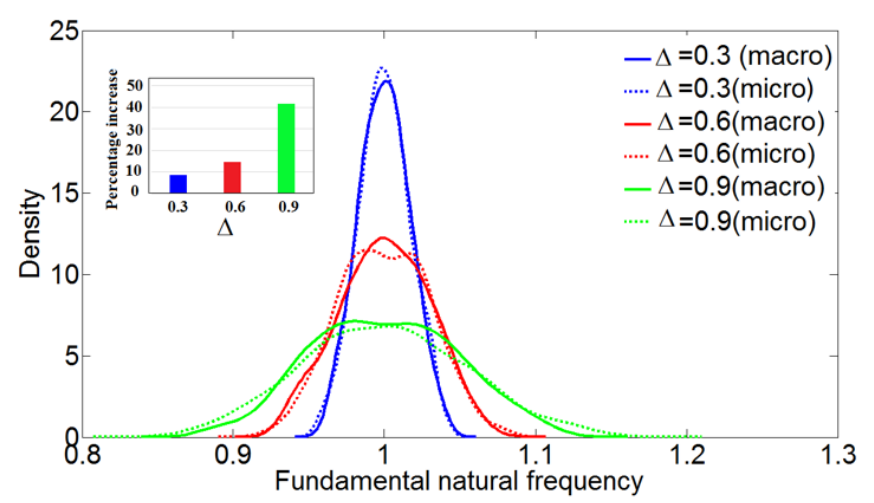

(a)

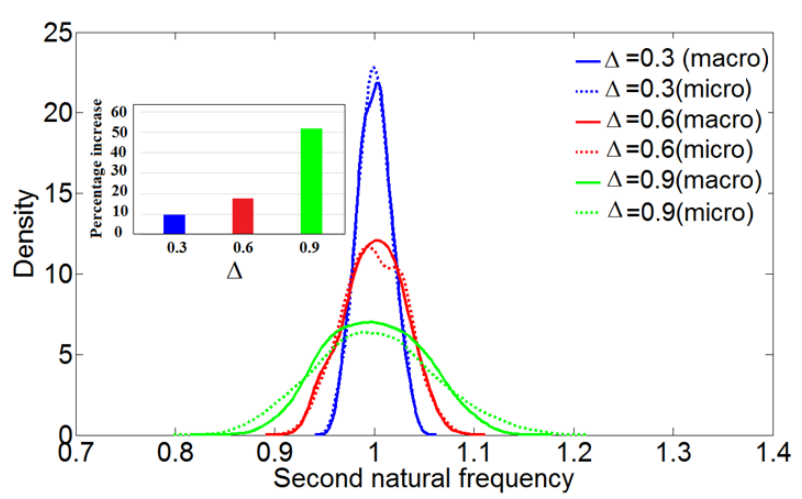

(b)

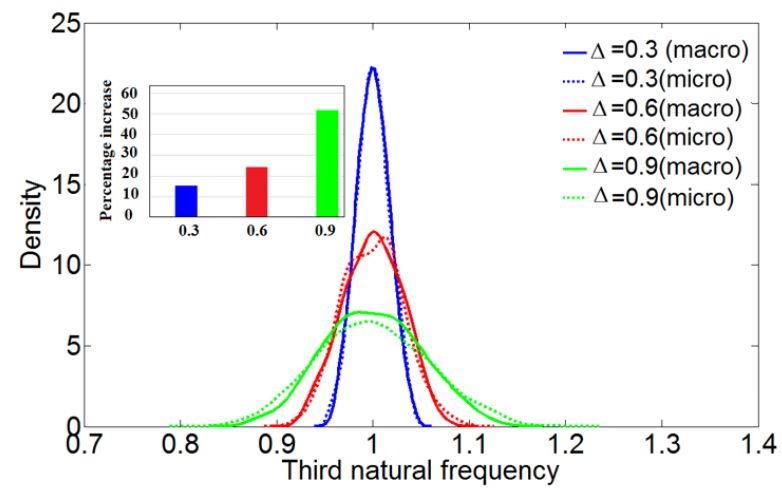

(c)

Fig. 11 Probability density function (pdf) plots for macro $\left(g_{\text {macro }}^{C}\right)$ and micro $\left(g_{\text {micro }}^{C}\right)$ mechanical analyses of natural frequencies for different degree of stochasticity $(\Delta)$. Normalized results are used with respect to the corresponding deterministic values for plotting the pdfs. The bar plots in the inset indicate the percentage increase of stochastic bounds for a micromechanical analysis with respect to the macromechanical analysis.

Figure 15 presents the relative coefficient of variation (RCOV) for individual stochastic effect of different uncertain input parameters considering both the macro $\left(g_{\text {macro }}^{I}\right)$ and micro $\left(g_{\text {macro }}^{I}\right)$ mechanical analyses. To obtain these figures, MCS are carried out for the variation of each of the micro and macro mechanical material parameters individually. The figures can provide a clear understanding regarding the relative sensitivity of various stochastic system parameters (input) to the output natural frequencies in the macro and micro mechanical analyses. For stochasticity in macro-mechanical material properties, it is observed that mass density, longitudinal Young's modulus and transverse Young's modulus (in decreasing order of sensitivity) are most sensitive to the first three natural frequencies, while the shear 


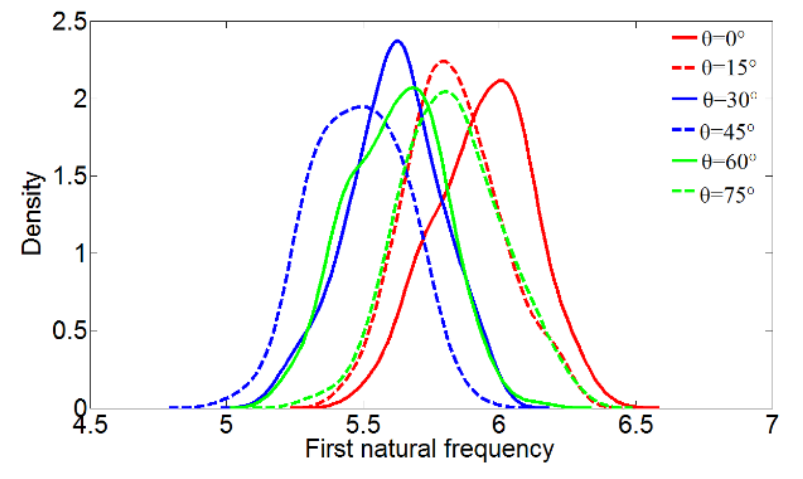

(a)

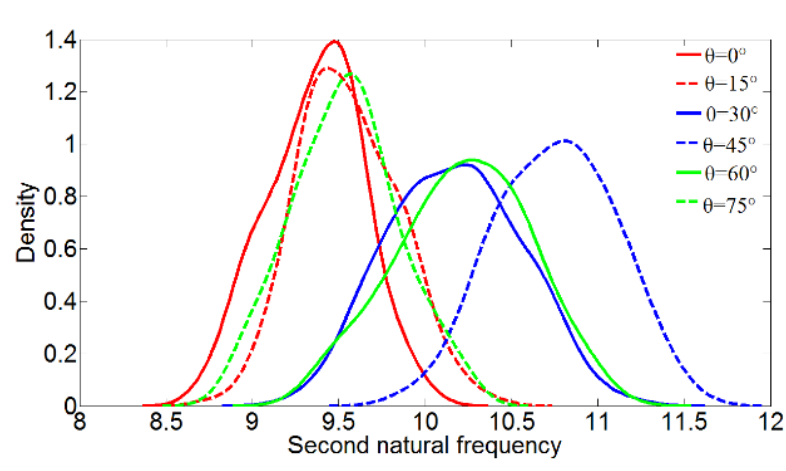

(b)

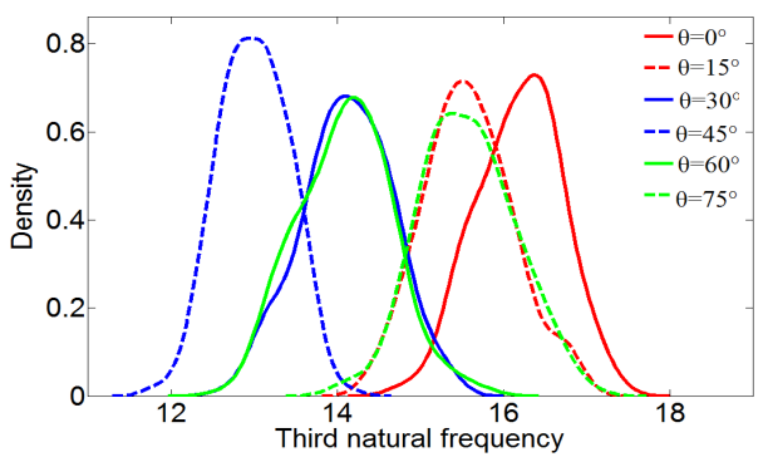

(c)

Fig. 12 Probability density function (pdf) plots for natural frequencies considering different ply orientation angle $(\theta)$ for stochasticity in micro-mechanical properties $\left(g_{\text {micro }}^{C}\right)$

moduli and Poisson's ratio are comparatively less sensitive. For the micro-mechanical analysis, it is found that the most sensitive system parameters (stochastic input) according to decreasing order of sensitivity are mass density of fibre, longitudinal Young's modulus of fibre and volume fraction, while transverse Young's modulus of fibre, Poisson's ratio of fibre and shear modulus of fibre are the least sensitive parameters. Two different forms of analyses, as carried out here considering macro and micro mechanical material properties, render an in-depth understanding regarding the relative influence of various stochastic input parameters (source-uncertainty). For example, the macromechanical analysis (refer to figure 15(a)) shows that mass density of composites is the most sensitive parameter for low frequency vibration modes; however, the micromechanical analysis (refer to figure 15(b)) provides information in more depth showing that mass density of fibre is the most sensitive parameter. Outcomes of such sensitivity analyses serve as an important guideline for efficient uncertainty quantification (including dimensionality reduction) and subsequent analysis/ design and quality control of input parameters. 


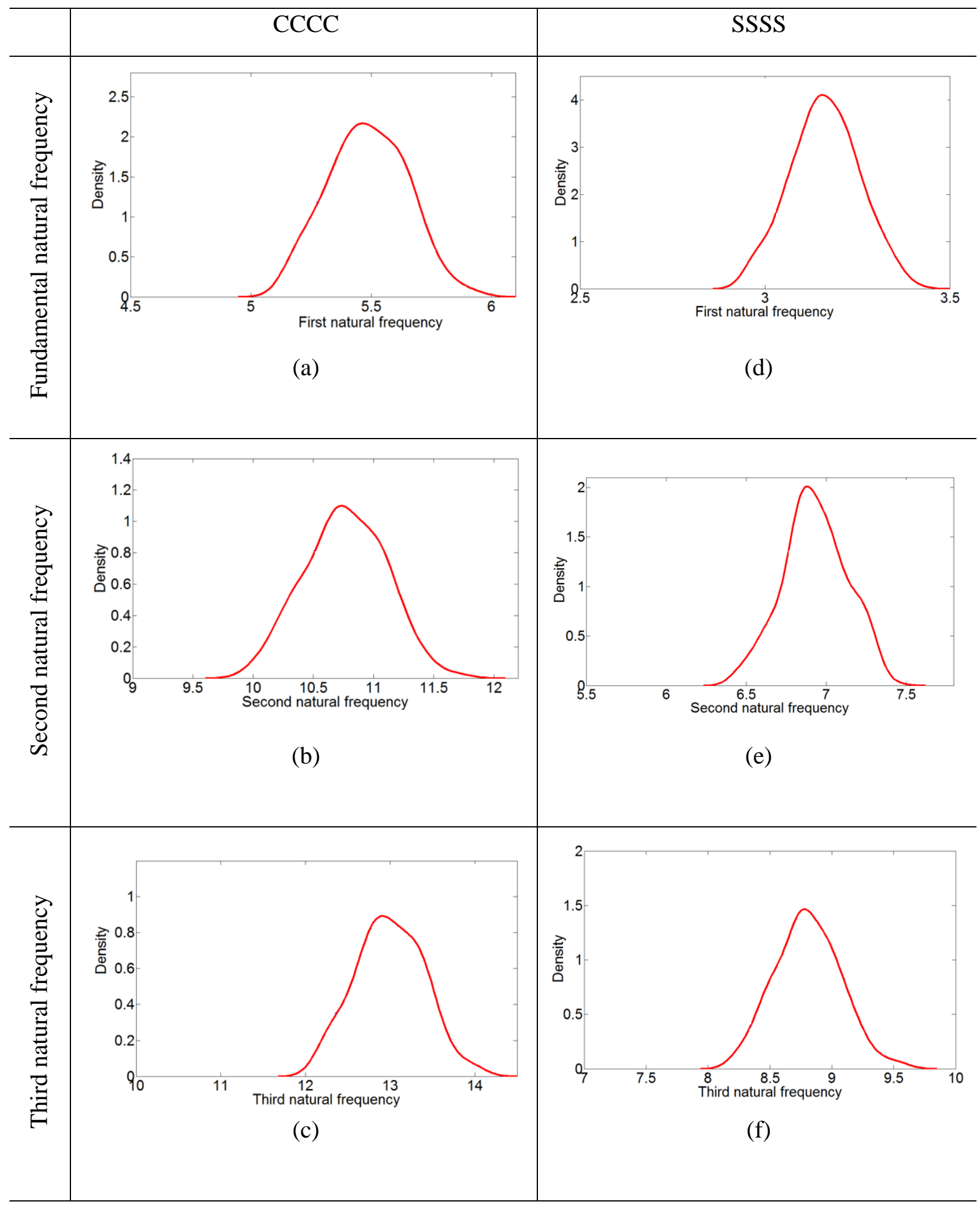

Fig. 13 (a-c) Probability density function (pdf) plots of natural frequencies for micro mechanical analysis ( $g_{\text {micro }}^{C}$ ) considering clamped boundary condition $(\mathrm{CCCC})(\mathrm{d}-\mathrm{f})$ Probability density function (pdf) plots of natural frequencies for micro mechanical analysis $\left(g_{\text {micro }}^{C}\right)$ considering simply supported boundary condition (SSSS) 


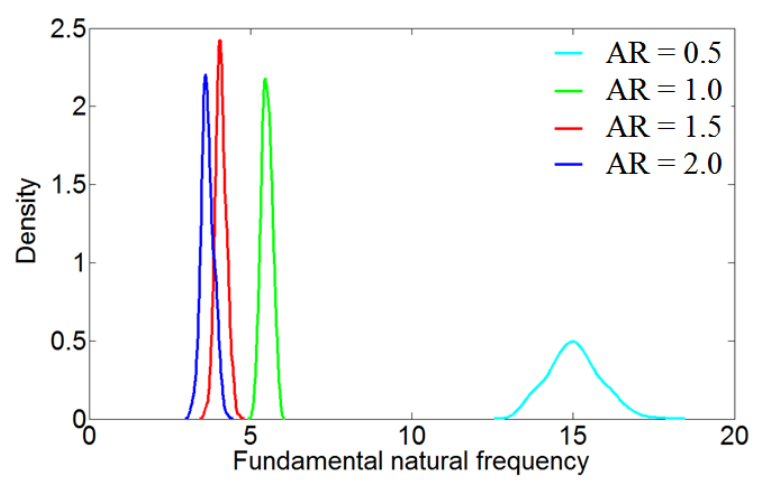

(a)

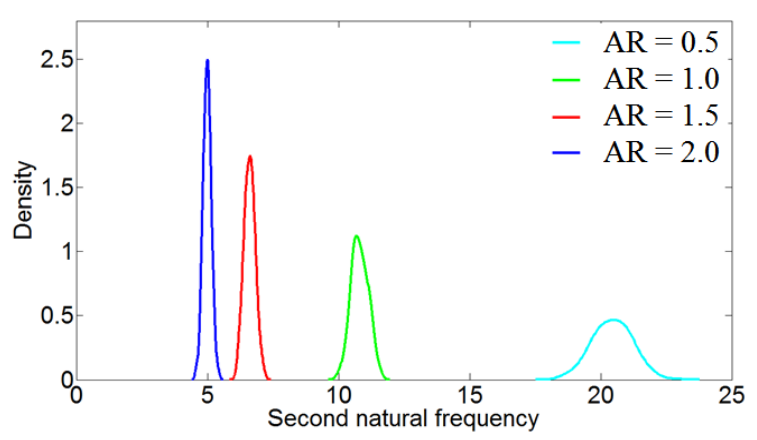

(b)

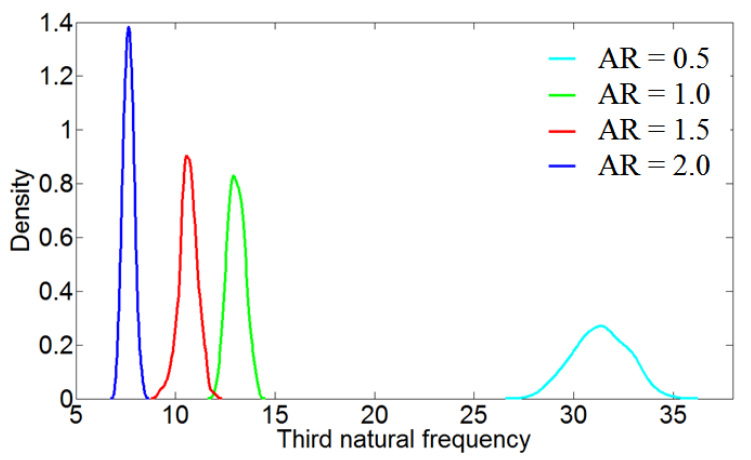

(c)

Fig. 14 Probability density function (pdf) plots of natural frequencies for different values of aspect ratios $(\mathrm{AR})$ considering micro-mechanical properties $\left(g_{\text {micro }}^{C}\right)$

The random field of the stochastic micro and macro mechanical material properties depend on the correlation length considered in the analysis. We have investigated the effect of correlation length on the first three modes of vibration. Depending on the value of correlation length, the random field of all the micro and macro mechanical properties would vary; representative plots are presented in figure 16 showing the spatial distribution of micro and macro mechanical material properties concerning the longitudinal Young's modulus for a single random realization considering different values of correlation length. It can be noted that two extreme cases can be realized when the correlation length tends to the upper and lower limits. The system becomes a randomly homogenous system (analogous to the random variable based approach) when the correlation length is very high, while it becomes an uncorrelated randomly inhomogeneous system when the correlation length is very low. The typical probability distribution of a representative micromechanical property $\left(E_{1 f}\right)$ and a macromechanical property $\left(E_{1}\right)$ for a randomly chosen SRVE having three different laminae is shown in figure 17 considering a correlation 


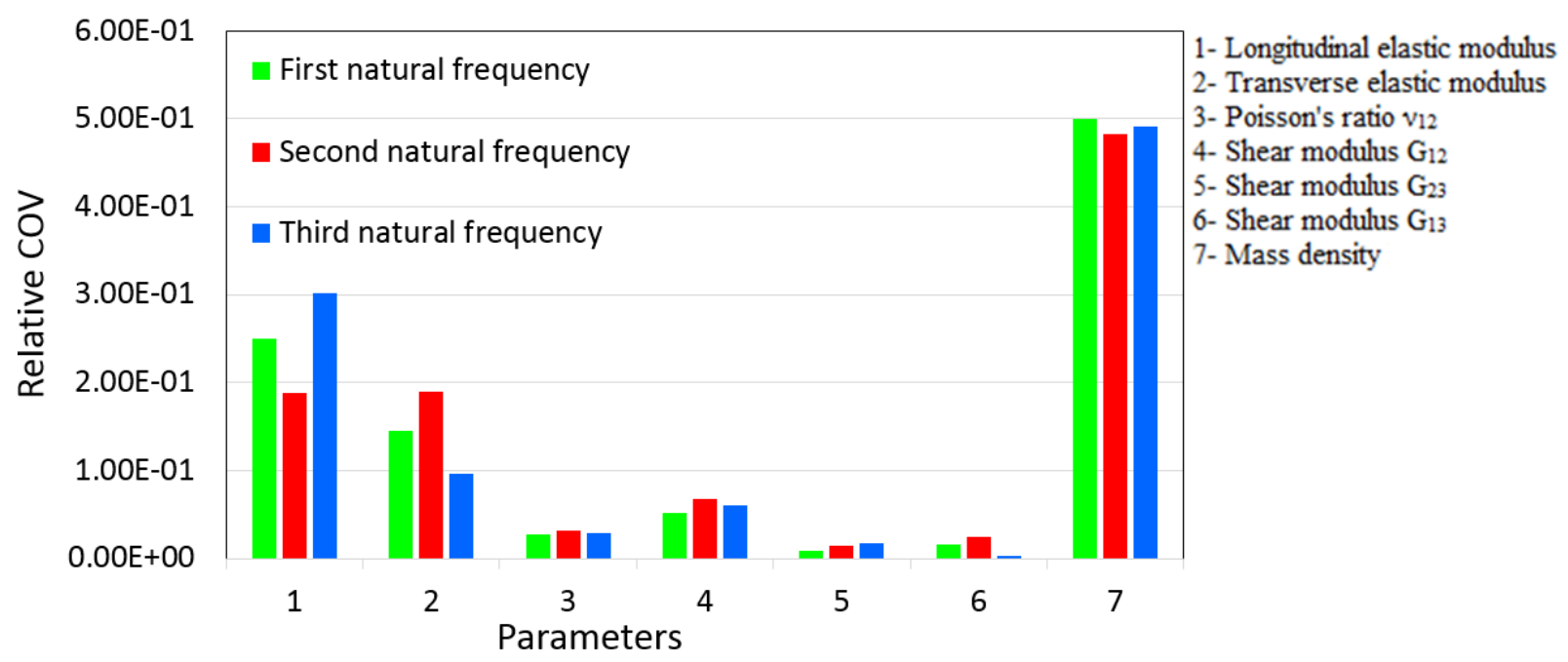

(a)

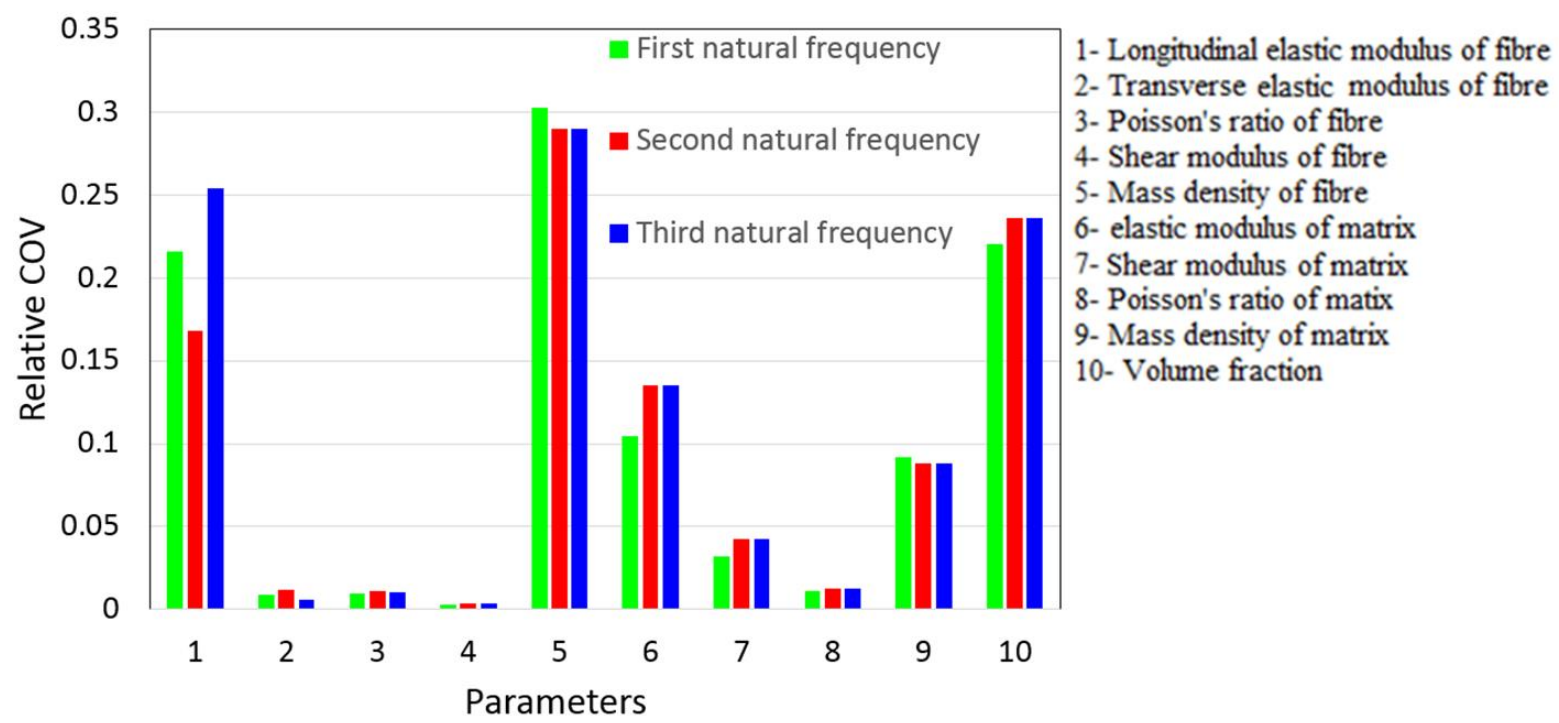

(b)

Fig. 15 (a) Relative coefficient of variation (RCOV) for the natural frequencies considering macromechanical material properties (b) Relative coefficient of variation (RCOV) for the natural frequencies considering micro-mechanical material properties

length of $1 / 50$. The effect of correlation length on the probability density function plots of the first three natural frequencies is presented in figure 18, wherein a clear difference is noticed between the lower and higher values of correlation lengths. This, in turn indicates the difference in the probabilistic characteristics of the natural frequencies corresponding to random field (lower values of correlation length) and random variable (higher value of correlation length) approach. The figure shows that the mean and response bound decrease in case of the random field based modelling of source-uncertainty compared to the random variable based modelling. 


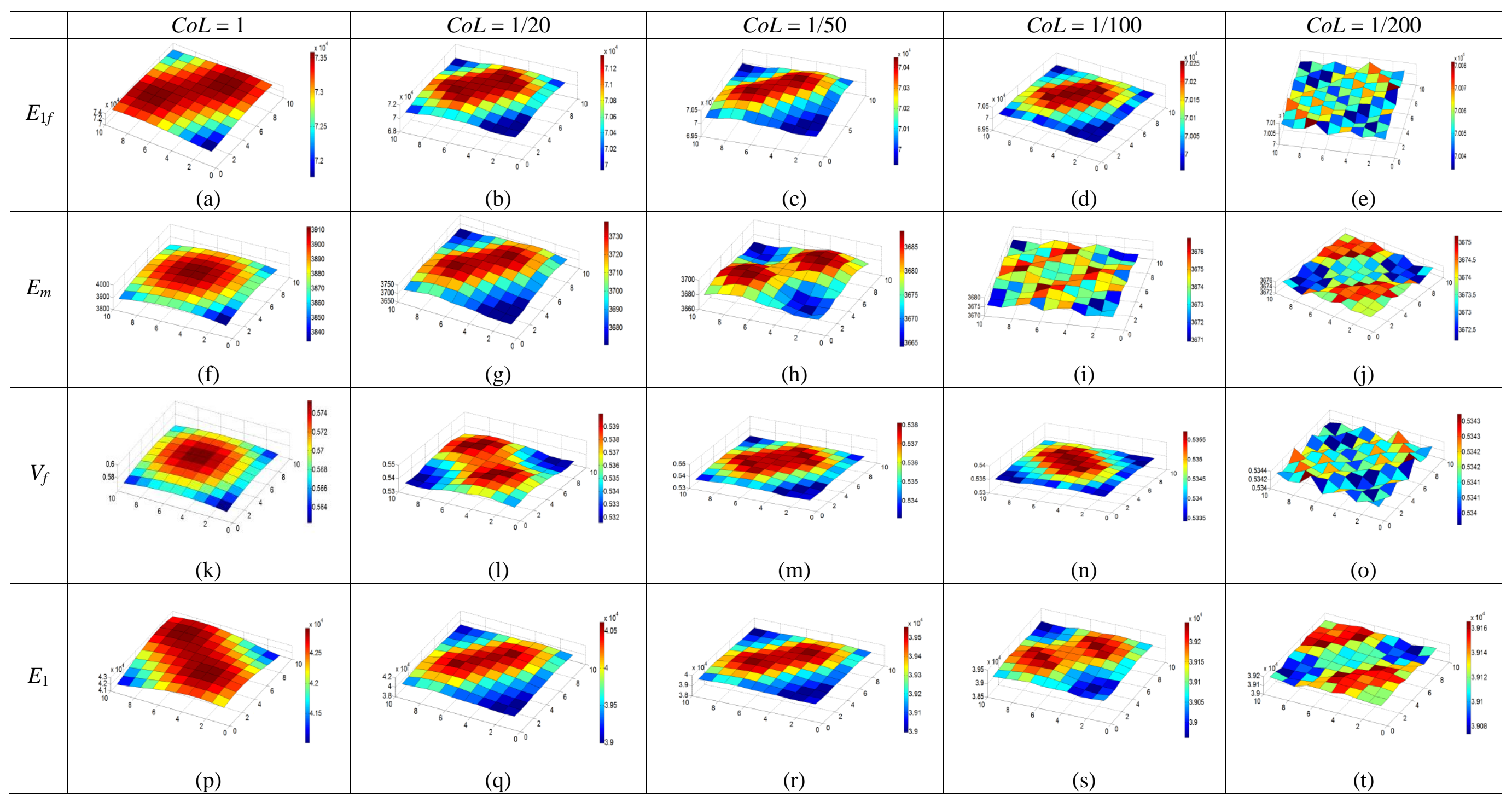

Fig. 16 Representative plots showing the spatial distribution of micro and macro mechanical properties concerning the longitudinal Young's modulus for a random realization considering different values of correlation length $(C o L)$. Here $\mathrm{X}$ and $\mathrm{Y}$ axes in the figures are the two spatial directions of a lamina, while the $\mathrm{Z}$ axis shows the value of corresponding material property. 


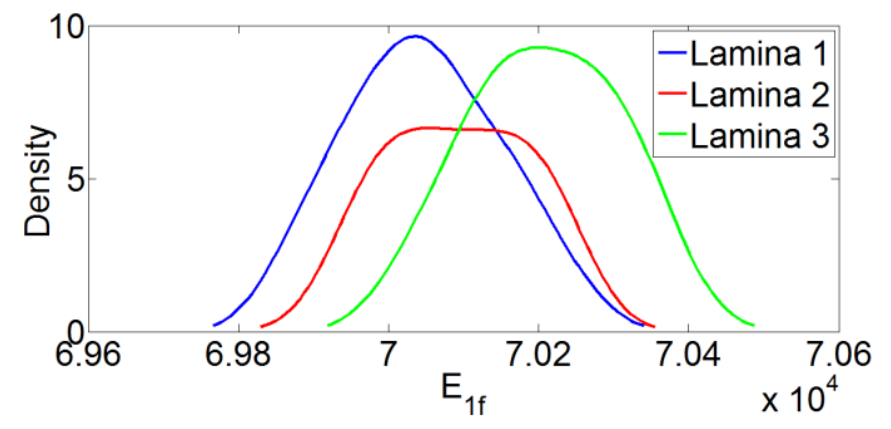

(a)

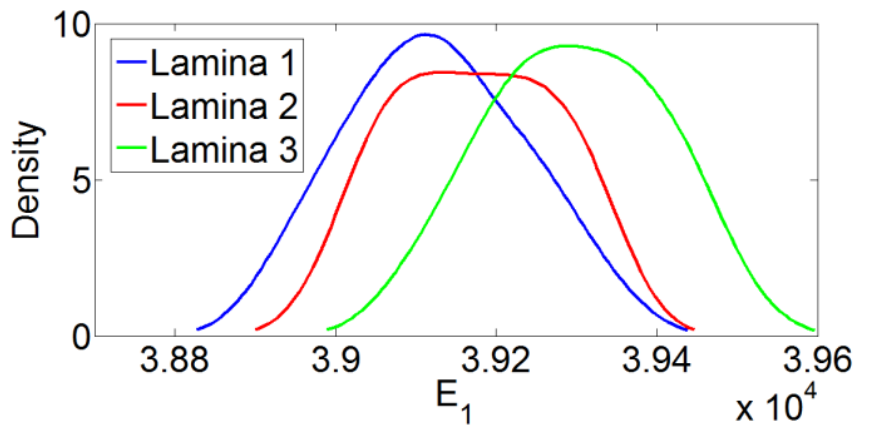

(b)

Fig. 17 Probability distribution of a representative micromechanical property $\left(E_{1 \mathrm{f}}\right)$ and a macromechanical property $\left(E_{1}\right)$ for a randomly chosen SRVE having three different laminae considering a correlation length of $1 / 50$

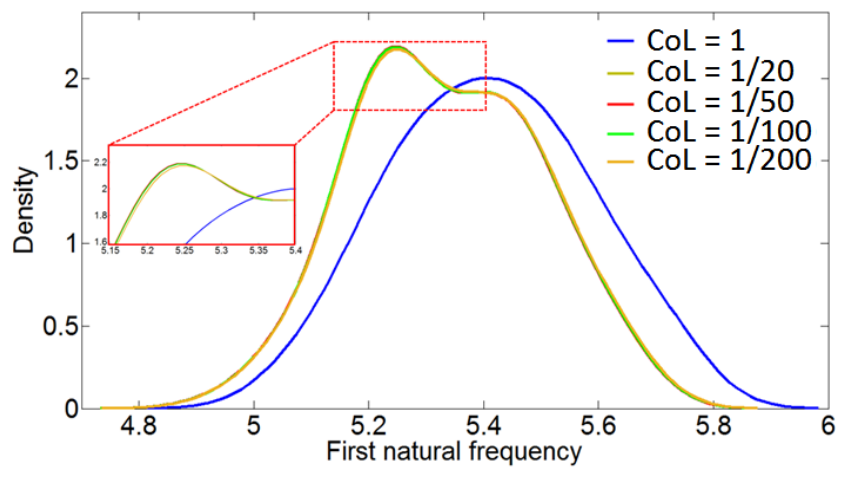

(a)

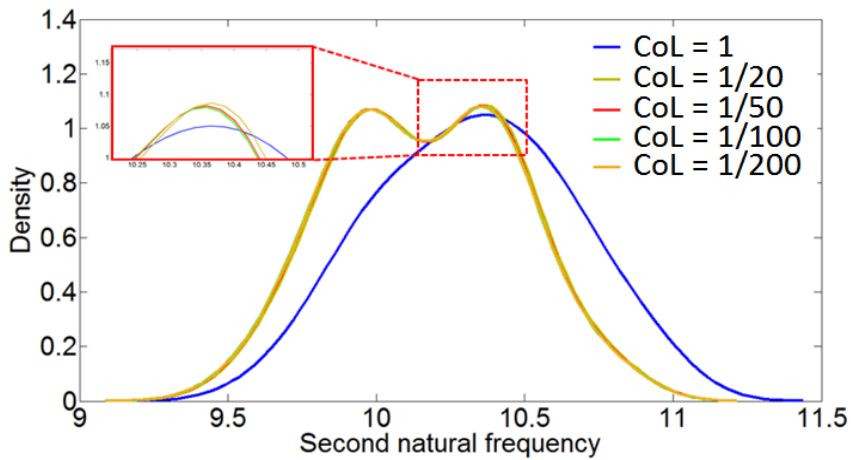

(b)

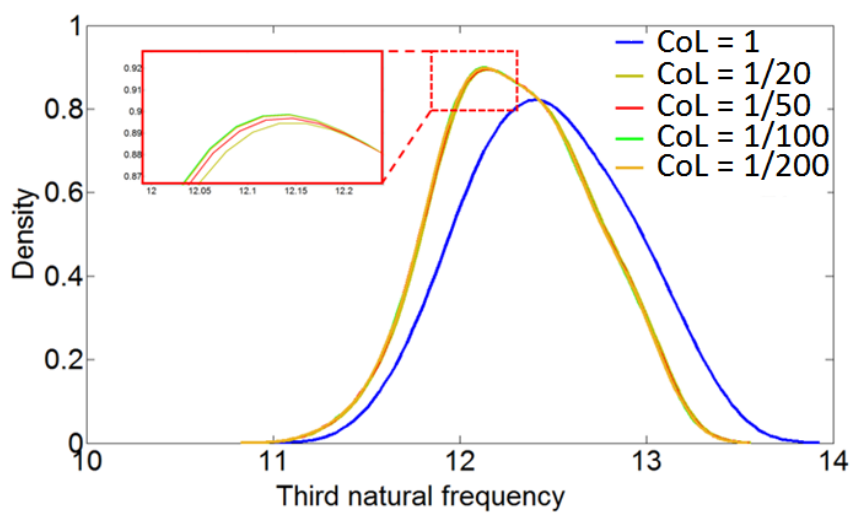

(c)

Fig. 18 Effect of correlation length $(\mathrm{CoL})$ on the probability distribution of the first three natural frequencies of a composite laminate

The effect of stochasticity in micro and macro mechanical material properties are studied on the vibration mode shapes considering two different boundary conditions (SSSS and CCCC). The results are presented in figure 19 for first three modes of vibration. Stochastic mode shapes for first three modes of 


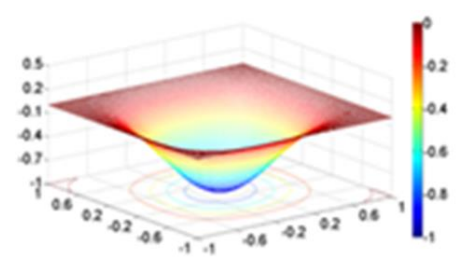

(a)

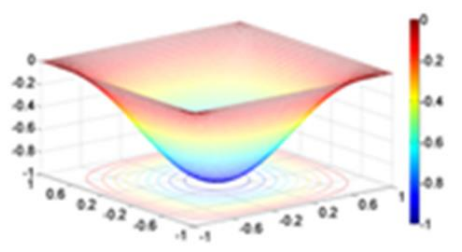

(d)

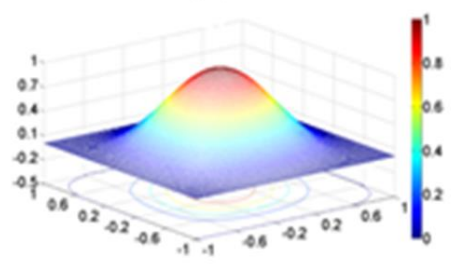

(g)

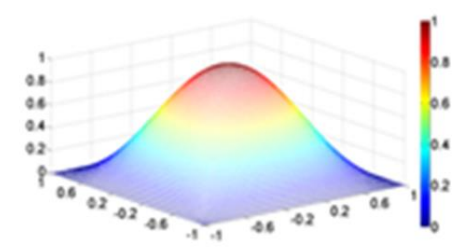

(j)

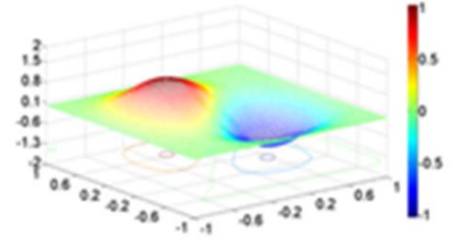

(b)

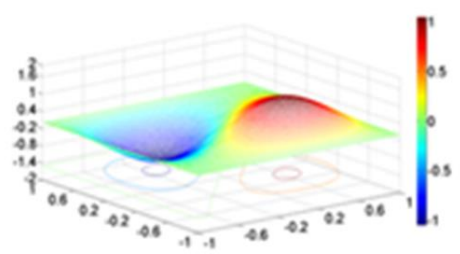

(e)

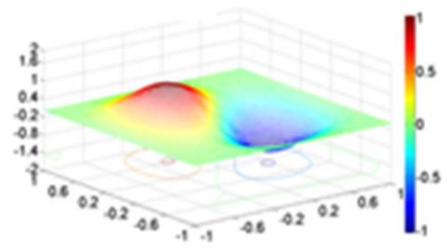

(h)

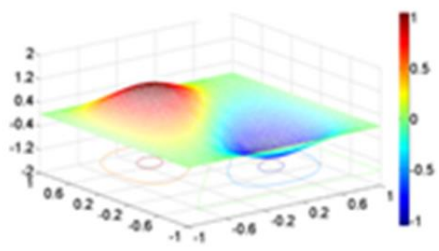

(k)

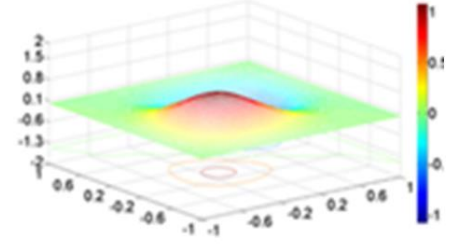

(c)

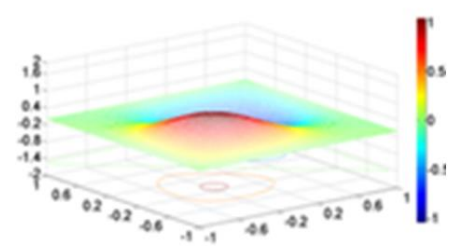

(f)

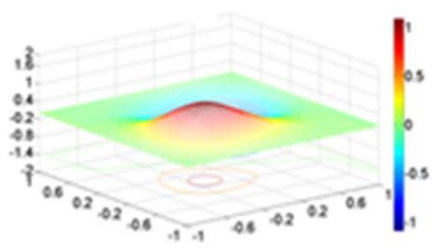

(i)

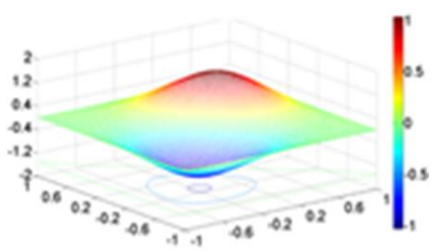

(1)

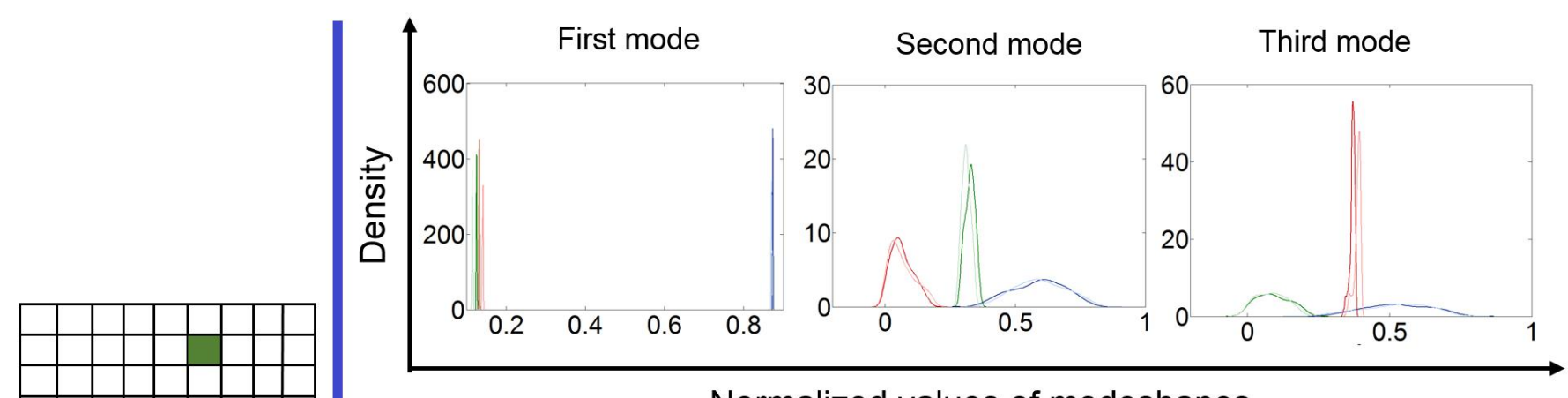

Normalized values of modeshapes

(m)

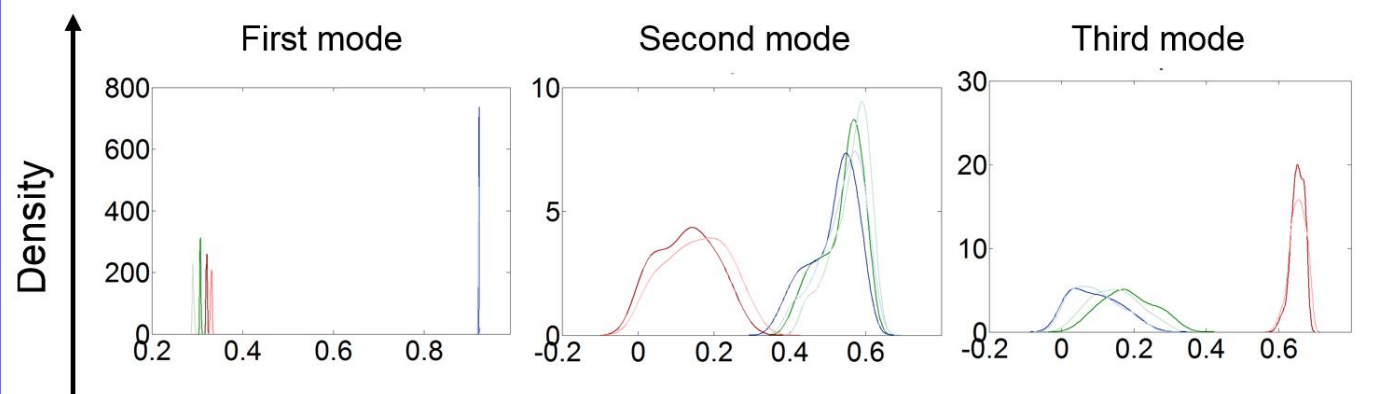

Normalized values of modeshapes

(n)

Fig. 19 Stochastic modeshapes and representative probability distribution of the normalized eigenvectors 
vibration considering a single random realization are presented in case of: a clamped (CCCC) composite plate with stochasticity in the macromechanical $\left(g_{\text {macro }}^{C}\right)$ properties (refer to figure $19(\mathrm{a}-\mathrm{c})$ ), a simply supported (SSSS) composite plate with stochasticity in the macromechanical $\left(g_{\text {macro }}^{C}\right)$ properties (refer to figure 19(d-f)), a clamped (CCCC) composite plate with stochasticity in the micromechanical $\left(g_{\text {micro }}^{C}\right)$ properties (refer to figure 19(g-i)) and a clamped (CCCC) composite plate with stochasticity in the micromechanical $\left(g_{\text {micro }}^{C}\right)$ properties (refer to figure $19(\mathrm{j}-1)$ ). From the mode shapes presented in figure 19(a - 1), it can be observed that the basic global pattern of the stochastic mode shapes remains similar to the corresponding deterministic case. However, the value of normalized eigenvectors becomes stochastic in nature for each of the elements in the composite plate. Probability distribution of the normalized eigenvectors of first three vibration modes for the elements indicated in figure 19(o) are shown considering a clamped (CCCC) boundary condition (refer to figure 19(m)) and a simply supported (SSSS) boundary condition (refer to figure 19(n)). The results for micro and macro mechanical analyses are shown using lighter and darker shades of respective colours indicated in figure 19(o). It can be noticed that the probability density function plots depend significantly on the type of analysis (micro and macro mechanical) and location of the element under consideration.

\subsection{Stochastic stability analysis}

\subsubsection{Validation and convergence study}

The FE code and the surrogate model are validated first for analysing the buckling loads similar to the case of dynamic analysis as discussed in section 5.1.1. The results of convergence study and validation of the finite element code of a composite plate is furnished in Table 4, wherein the nondimensional first buckling load is validated with the results available in scientific literature. Based on the results presented in Table 4, a mesh size of $9 \times 9$ is found to be adequate for the finite element model. The optimum number of samples (drawn from Sobol sequence) to form the surrogate models of buckling loads are decided based on the comparative performance (four different statistical parameters: minimum value, maximum value, mean value and standard deviation) with respect to direct MCS. The results of convergence study for macro and micro mechanical analyses showing the values of absolute error with 
Table 4 The convergence study of uniaxial buckling load of four layer $\left(0^{\circ} / 90^{\circ} / 90^{\circ} / 0^{\circ}\right)$ simply supported SSSS rectangular laminates $\left(\bar{N}=\bar{N}_{x x} b^{2} /\left(E_{2} h^{3}\right), \bar{N}_{x y}=0, \bar{N}_{y y}=0\right)$

\begin{tabular}{|c|c|c|c|}
\hline \multirow{2}{*}{$t / b$} & \multirow{2}{*}{ Mesh } & \multicolumn{2}{|r|}{ Buckling load } \\
\hline & & Present FEM & Reference results \\
\hline \multirow{6}{*}{0.001} & $5 \times 5$ & 23.3511 & \multirow{6}{*}{$\begin{array}{c}23.2928 \text { (Neves and Ferreira (2016)) } \\
23.463 \text { (Liew and Huang (2003)) }\end{array}$} \\
\hline & $6 \times 6$ & 23.3217 & \\
\hline & $7 \times 7$ & 23.3190 & \\
\hline & $8 \times 8$ & 23.2940 & \\
\hline & $9 \times 9$ & 23.2927 & \\
\hline & $10 \times 10$ & 23.2885 & \\
\hline
\end{tabular}

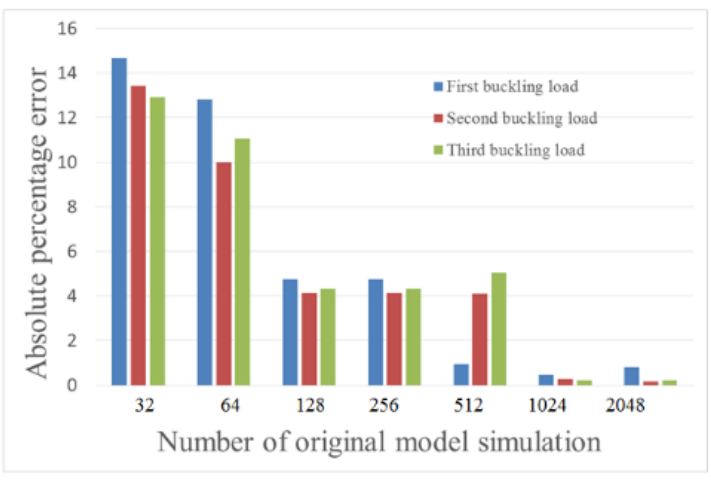

(a) Maximum

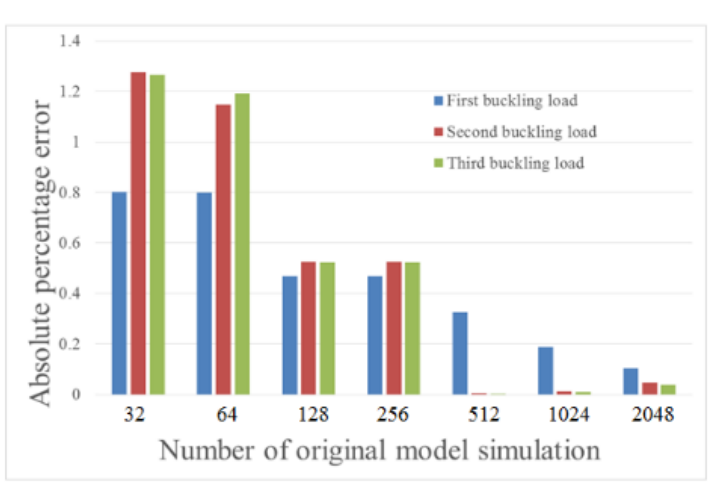

(c) Mean

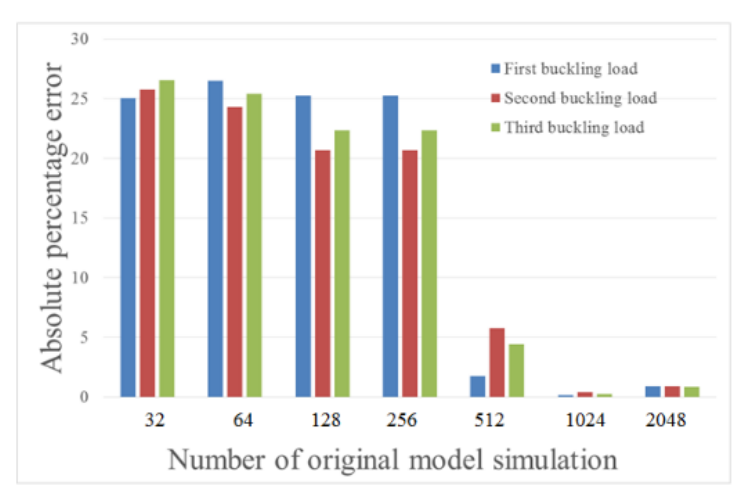

(b) Minimum

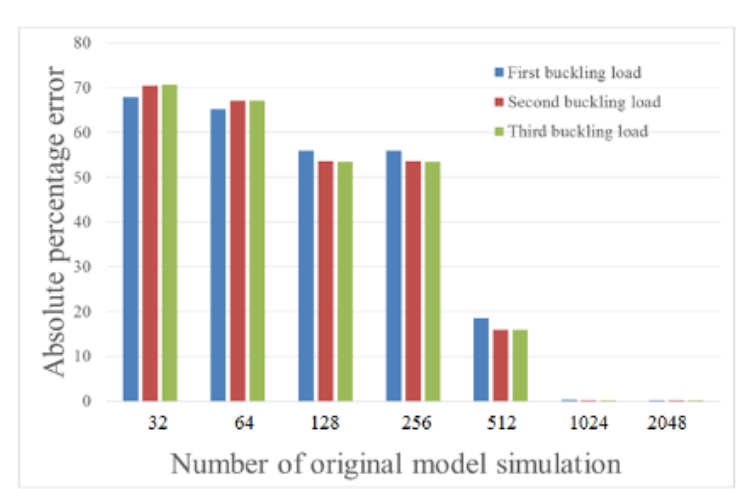

(d) Standard Deviation

Fig. 20 Absolute percentage error in minimum value, maximum value, mean value and standard deviation with respect to direct MCS for macro-mechanical analysis considering a coefficient of variation of 0.6 in the stochastic input parameters $\left(g_{\text {macro }}^{C}\right)$ 


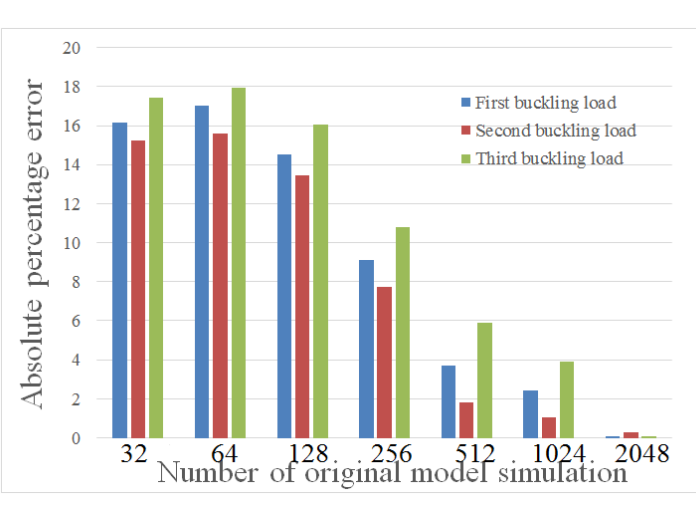

(a) Maximum

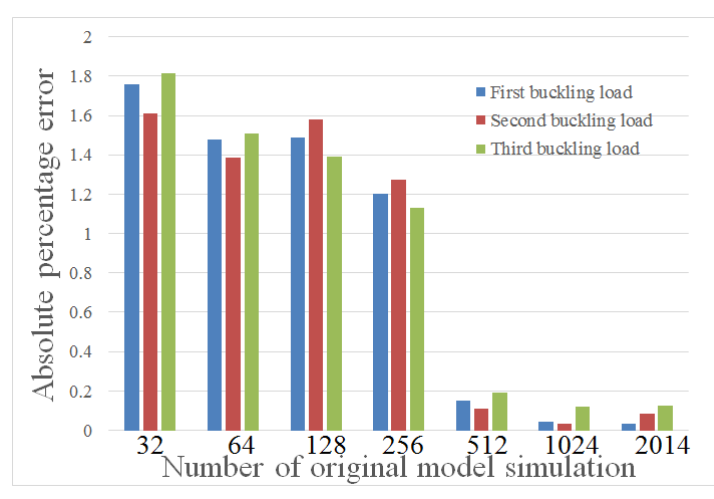

(c) Mean

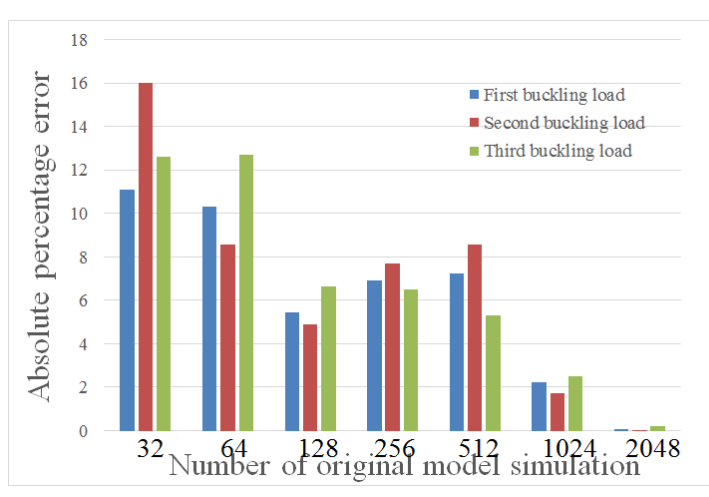

(b) Minimum

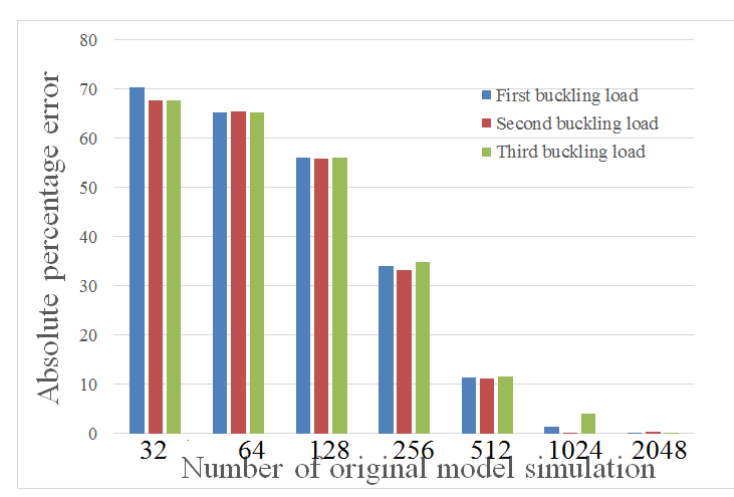

(d) Standard Deviation

Fig. 21 Absolute percentage error in minimum value, maximum value, mean value and standard deviation with respect to direct MCS for micro-mechanical analysis considering a coefficient of variation of 0.6 in the stochastic input parameters $\left(g_{\text {micro }}^{C}\right)$

different sample size are presented in figure 20 and 21. From the figures it is evident that a sample size of 1024 provides reasonably accurate results for predicting the buckling loads. To further examine the prediction capabilities of the surrogate models, scatter plots are presented for the macro and micro mechanical analyses in figures 22(a-c) and 23(a-c), respectively. Negligible deviation of the sample points from the diagonal line corresponding to the sample size of 1024 indicates the accuracy of prediction. The comparative probability density function plots on the basis of surrogate based MCS corresponding to the sample size of 1024 and direct MCS are presented for the macro and micro mechanical analyses in figures $22(\mathrm{~d}-\mathrm{f})$ and $23(\mathrm{~d}-\mathrm{f})$, respectively. A good agreement between the probabilistic descriptions of natural frequencies corroborates the accurate prediction capability of the surrogate models for further analyses.

In this section, we have presented the results of stochastic stability analysis considering the first three buckling modes for the sake of completeness (as presented in previous scientific literatures like Patel 


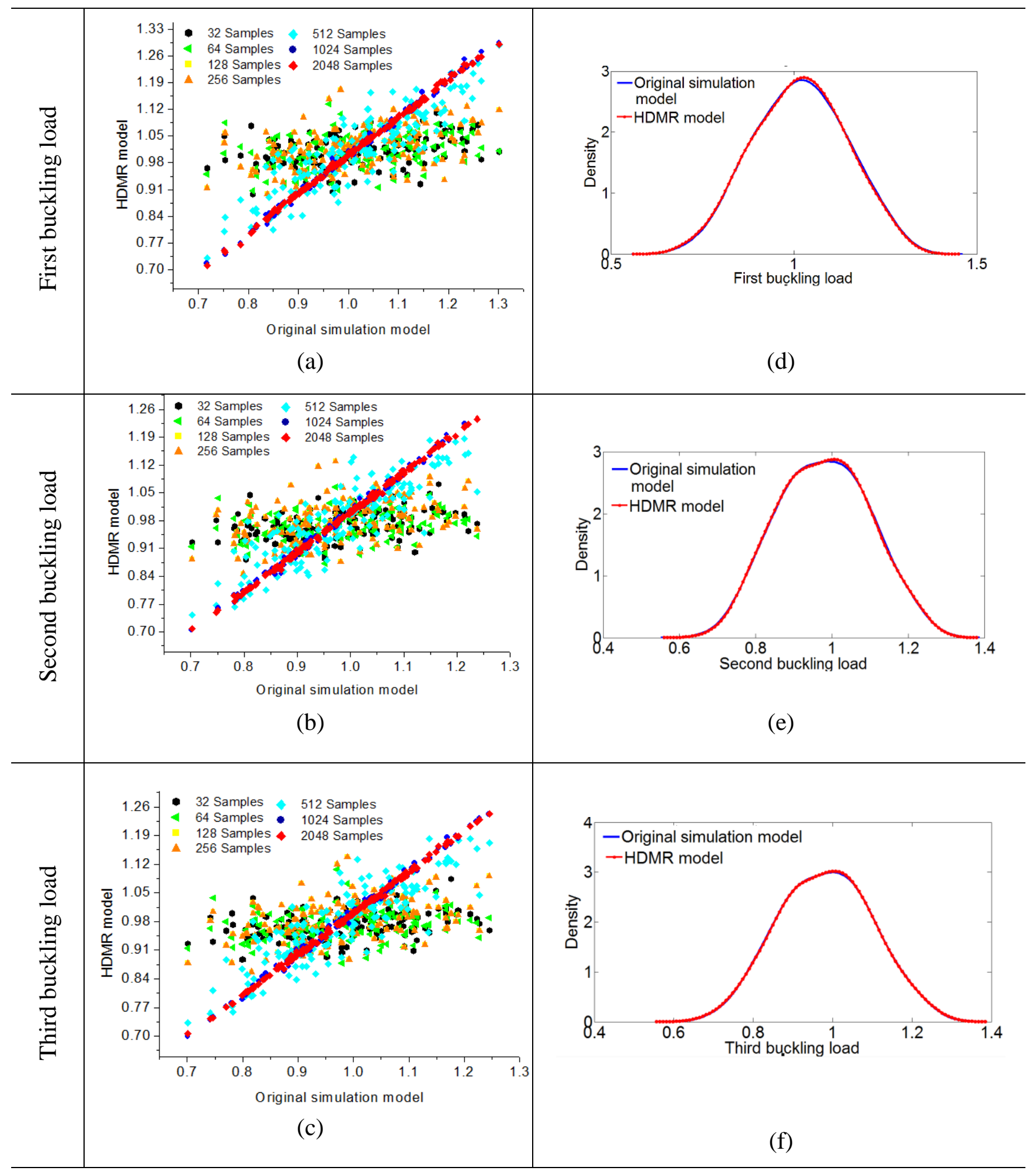

Fig. 22 (a-c) Scatter plots for HDMR based analysis (normalized on the basis of respective deterministic values) for various sample sizes of Sobol sequence considering the stochasticity in macro-mechanical attributes $\left(g_{\text {macro }}^{C}\right.$ ) with respect to direct MCS (with coefficient of variation of 0.6 in the stochastic input parameters) ; (d-f) Probability density function (pdf) of buckling loads (normalized with respect to the corresponding deterministic values) for macro-mechanical attributes $\left(g_{\text {macro }}^{C}\right)$ obtained by direct MCS and HDMR model based on a sample size of 1024 (with coefficient of variation of 0.6 in the stochastic input parameters) 


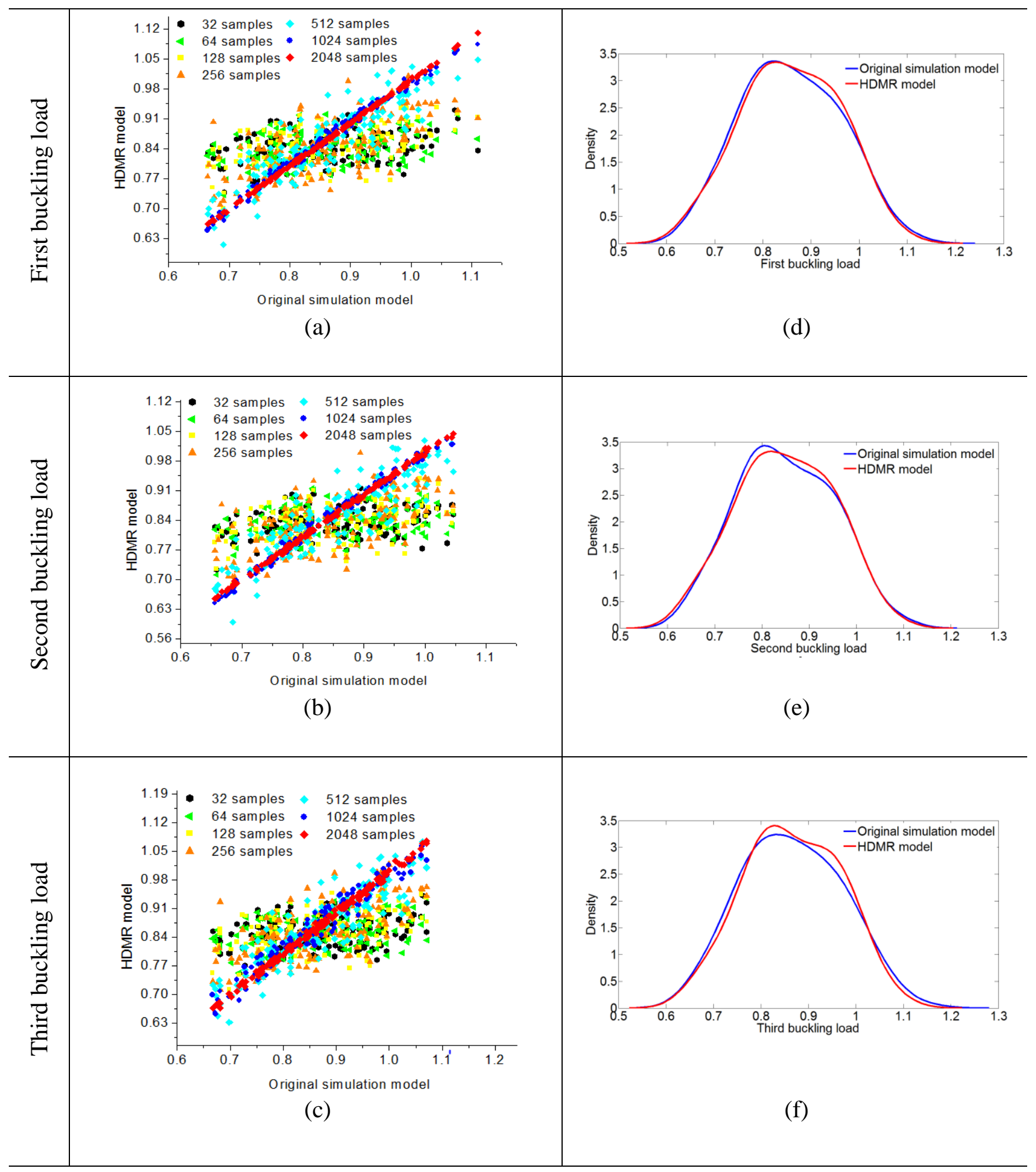

Fig. 23 (a-c) Scatter plots for HDMR based analysis (normalized on the basis of respective deterministic values) for various sample sizes of Sobol sequence considering the stochasticity in micro-mechanical attributes $\left(g_{\text {micro }}^{C}\right.$ ) with respect to direct MCS (with coefficient of variation of 0.6 in the stochastic input parameters) ; (d-f) Probability density function (pdf) of buckling loads (normalized with respect to the corresponding deterministic values) for micro-mechanical attributes $\left(g_{\text {micro }}^{C}\right)$ obtained by direct MCS and HDMR model based on a sample size of 1024 (with coefficient of variation of 0.6 in the stochastic input parameters) 


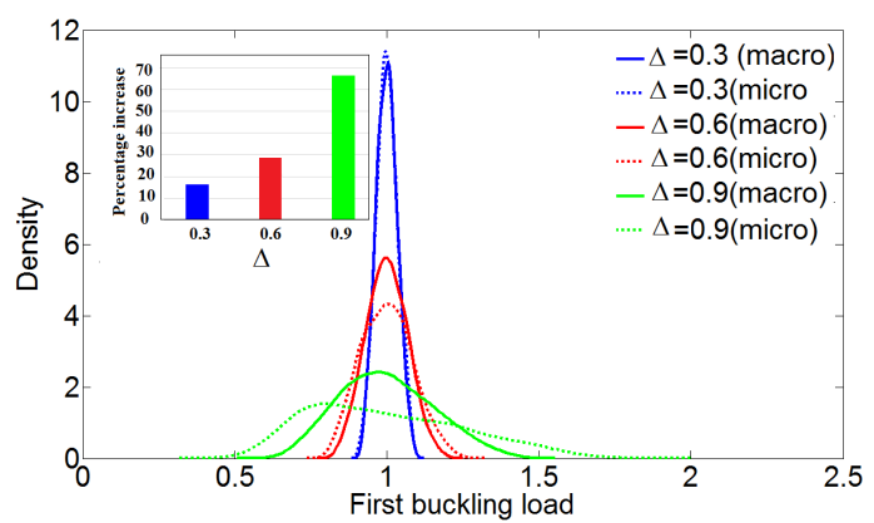

(a)

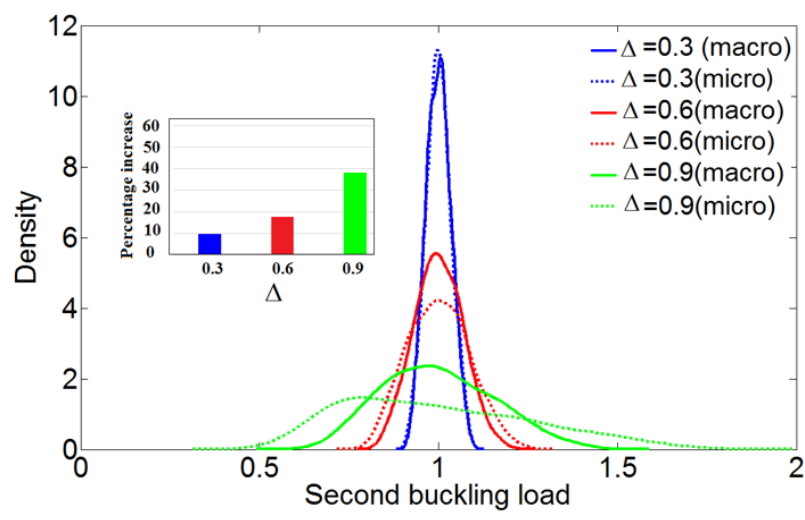

(b)

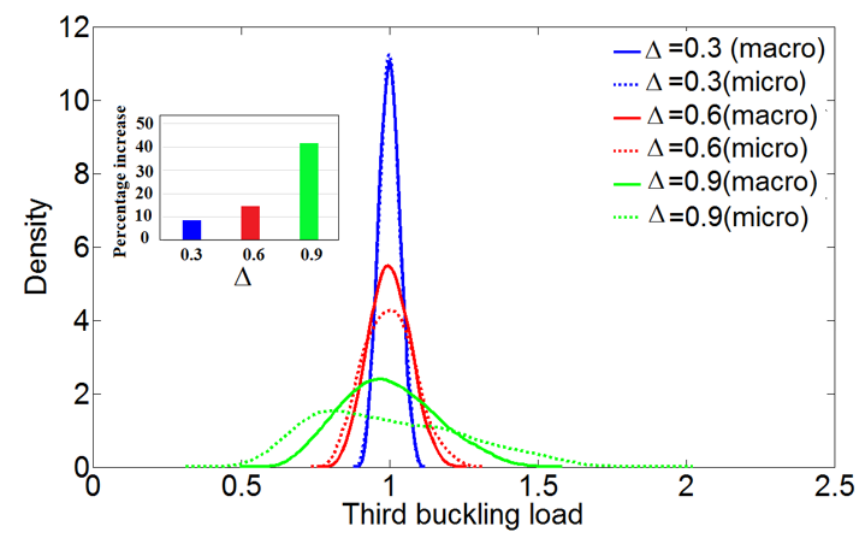

(c)

Fig. 24 Probability density function (pdf) plots for macro $\left(g_{\text {macro }}^{C}\right)$ and micro $\left(g_{\text {micro }}^{C}\right)$ mechanical analyses of first three buckling loads considering different degree of stochasticity $(\Delta)$. Normalized results are used with respect to the corresponding deterministic values for plotting the pdfs. The bar plots in the inset indicate the percentage increase of stochastic bounds for a micromechanical analysis with respect to the macromechanical analysis.

and Sheikh (2016)). Besides that, in case of stochastic stability analysis, the buckling loads are found to have a response bound (depending on the degree of stochasticity) with respect to the corresponding deterministic values. Thus there exists a possibility of overlap in the stochastic responses of the buckling loads corresponding to different modes of buckling resulting in a non-unique critical buckling mode with the minimum value of buckling load. For this reason, it is essential to consider higher buckling modes in case of stochasticity in the system parameters.

\subsubsection{Results of stochastic stability analysis}

Having the finite element model and the surrogate model validated, as shown in the preceding subsection, stochastic results are presented in this subsection for the first three modes of buckling for a 


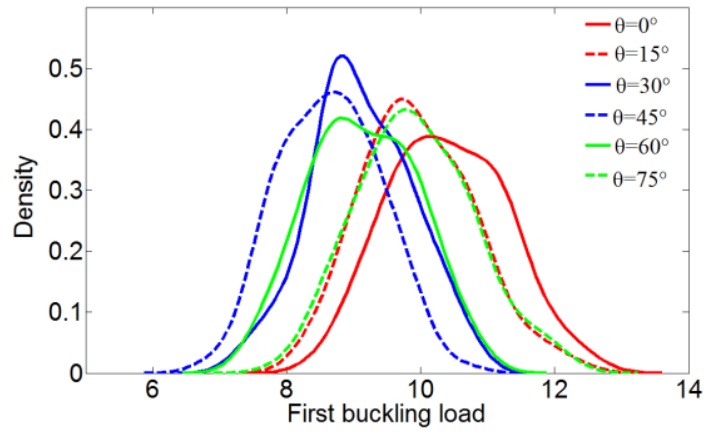

(a)

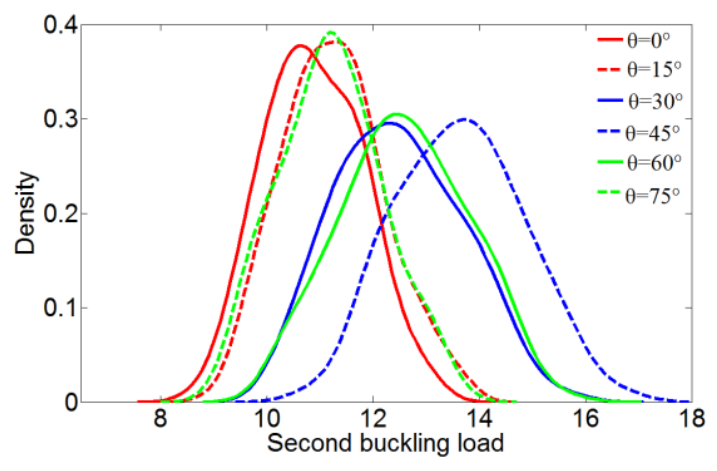

(b)

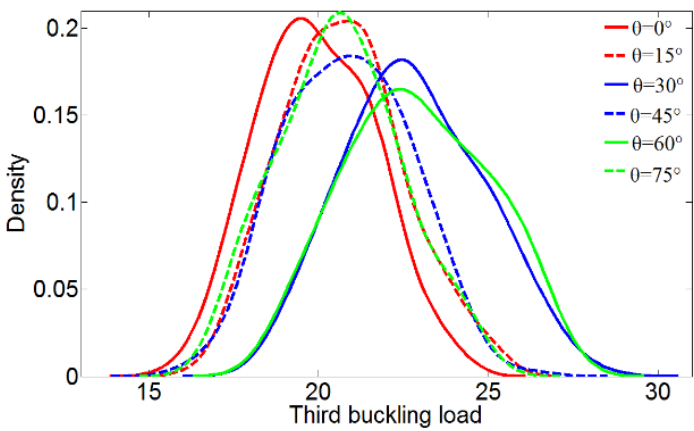

(c)

Fig. 25 Probability density function plots (pdf) for buckling loads considering different ply orientation angle $(\theta)$ for stochasticity in micro-mechanical properties $\left(g_{\text {micro }}^{C}\right)$

composite plate with correlated spatially varying material properies $\left(g_{\text {macro }}^{C}\right.$ and $\left.g_{\text {micro }}^{C}\right)$. Figure 24 shows the probabilistic descriptions for first three buckling loads considering different degree of stochasticity for macro-mechanical and micro-mechanical material properties. For both the micro and macro mechanical analyses, response bounds of the buckling loads are found to increase with the increasing degree of stochasticity. For a particular degree of stochasticity, similar to the case of dynamic analysis, it can be noticed that the response bound is higher in case of micromechanical analysis than that of the macromechanical analysis. Thus the probabilistic descriptions of the buckling loads differ from each other on the basis of the adopted type of analysis. A micromechanical analysis, which is more accurate for considering the source uncertainty at a more elementary level, shows substantially higher degree of variability in the global responses (both natural frequencies and buckling loads) due to the cascading effect in stochasticity. 


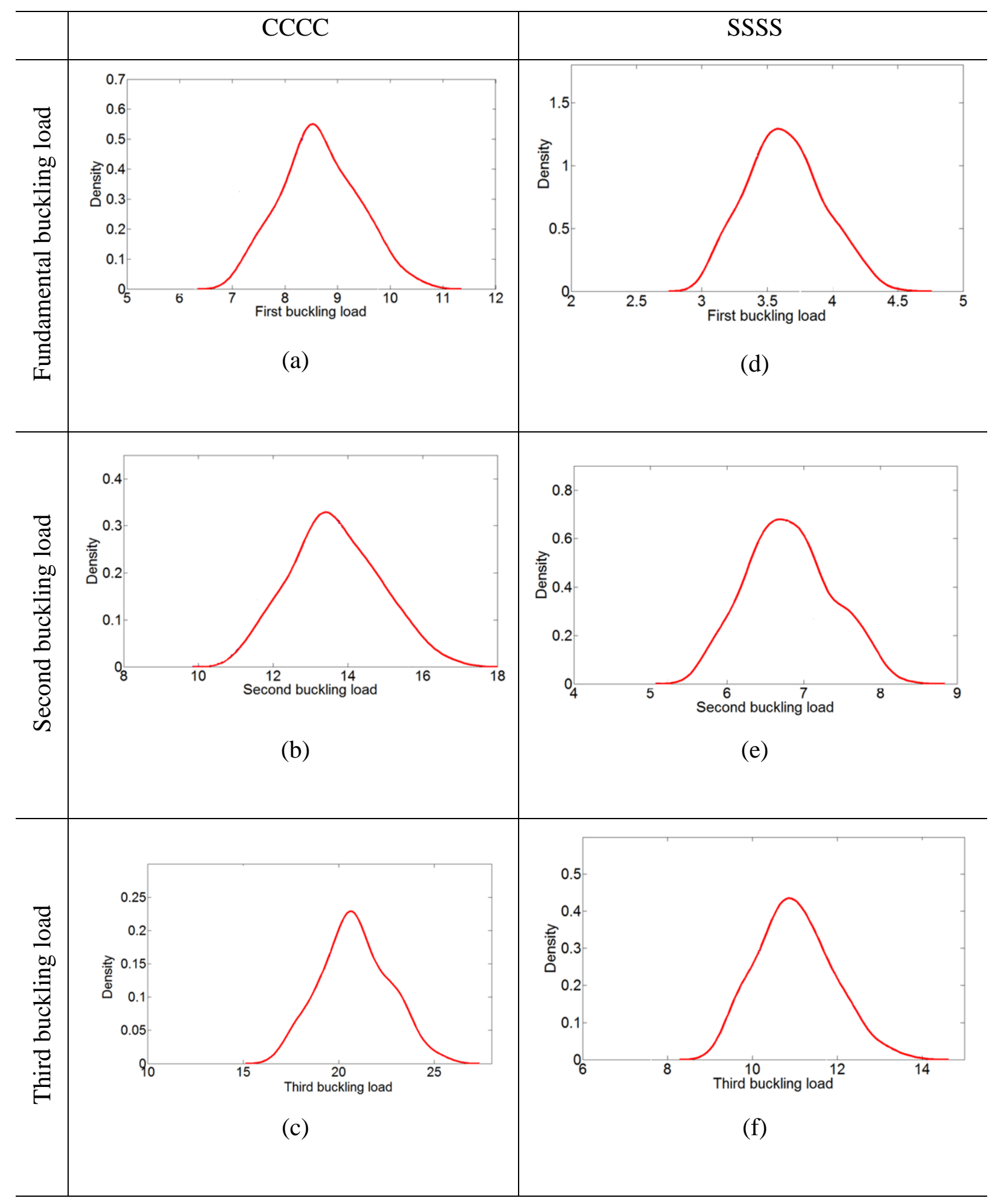

Fig. 26 (a-c) Probability density function (pdf) plots of buckling loads for micro mechanical analysis ( $g_{\text {micro }}^{C}$ ) considering clamped boundary condition (CCCC) (d-f) Probability density function (pdf) plots of buckling loads for micro mechanical analysis $\left(g_{\text {micro }}^{C}\right)$ considering simply supported boundary condition (SSSS) 


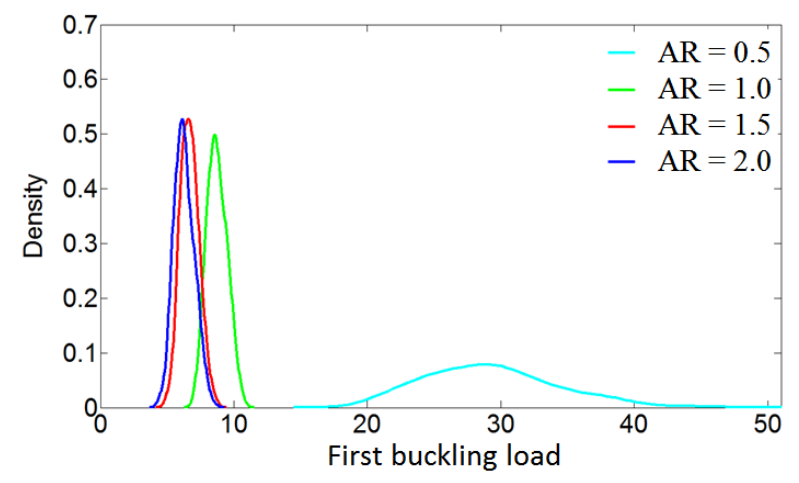

(a)

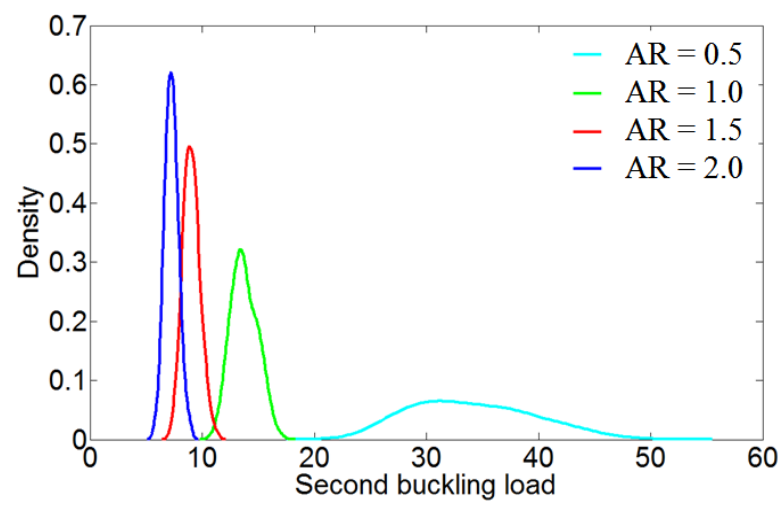

(b)

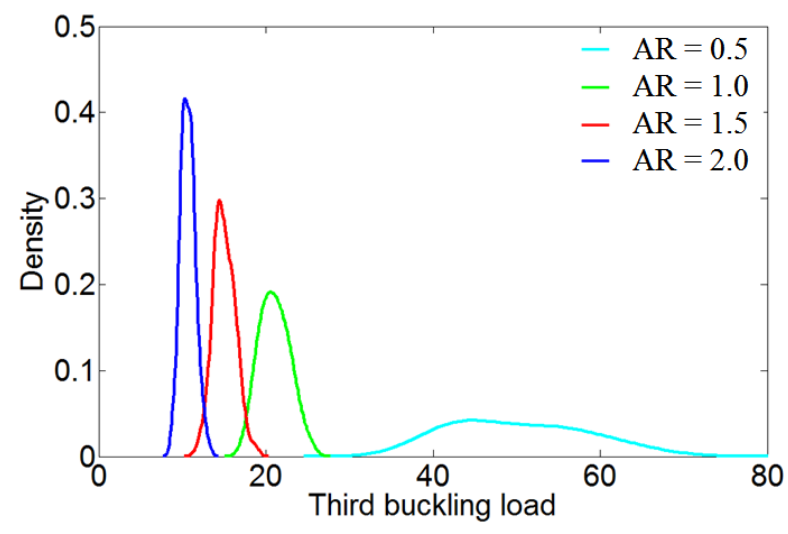

(c)

Fig. 27 Probability density function (pdf) plots of buckling loads for different values of aspect ratios (AR) considering micro-mechanical properties $\left(g_{\text {micro }}^{C}\right)$

The probabilistic descriptions of the first three buckling loads are presented in figure 25 for various laminate configurations considering the $[\theta /-\theta / \theta]$ family of composite for stochasticity in micro mechanical properties. Figure 26 shows the effect of different boundary conditions on stochastic buckling loads of laminated composite plates. Results are furnished for simply supported (SSSS) and fixed (CCCC) boundaries at all the four edges considering stochasticity in micro-mechanical properties. Figure 27 shows the effect of aspect ratio of the composite plates on stochastic buckling loads considering stochasticity in micro-mechanical properties. The buckling loads are found to reduce with the increase in aspect ratio.

Figure 28 presents the relative coefficient of variation (RCOV) considering individual probabilistic variation of the stochastic input parameters considering both the macro $\left(g_{\text {macro }}^{I}\right)$ and micro $\left(g_{\text {macro }}^{I}\right)$ mechanical analyses. To obtain these figures, MCS are performed for the variation of each of the macro and micro mechanical material parameters individually. The figures give a clear understanding regarding 


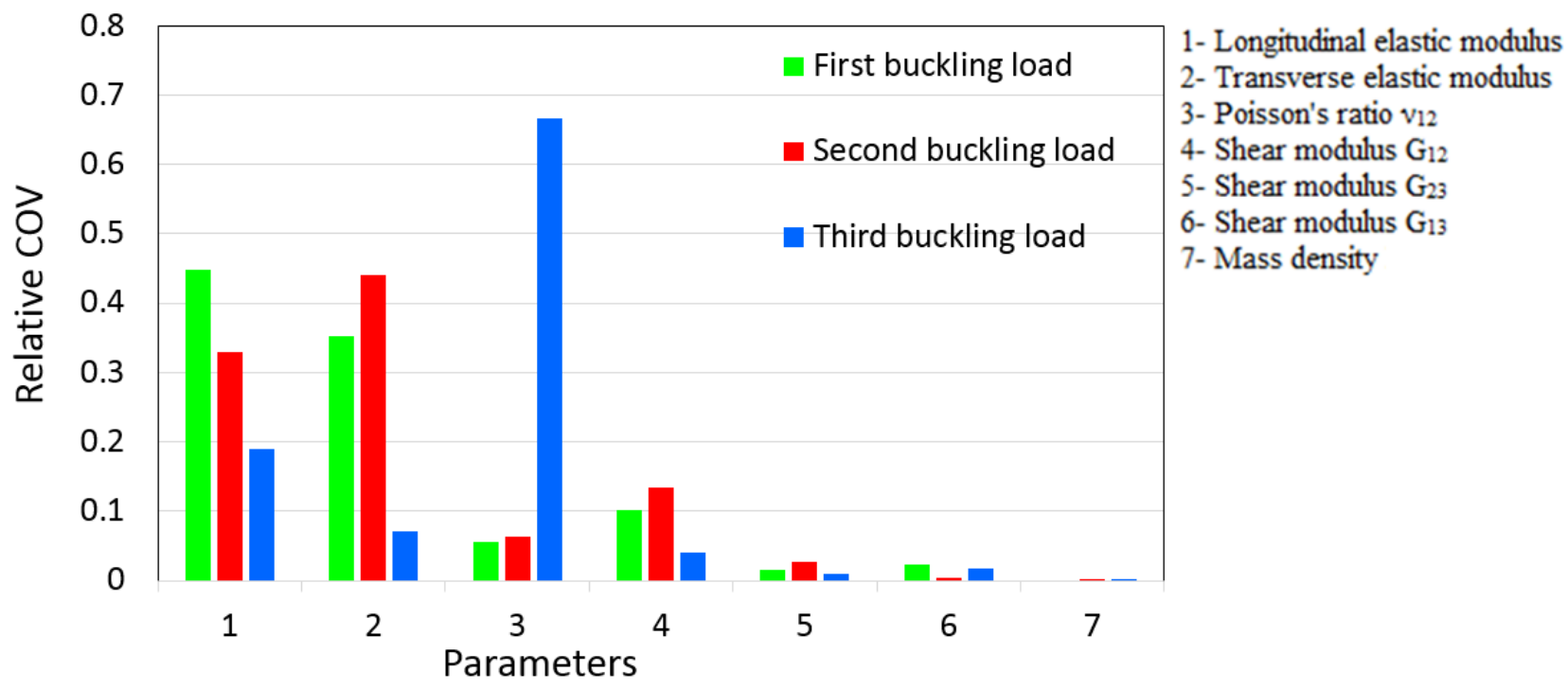

(a)

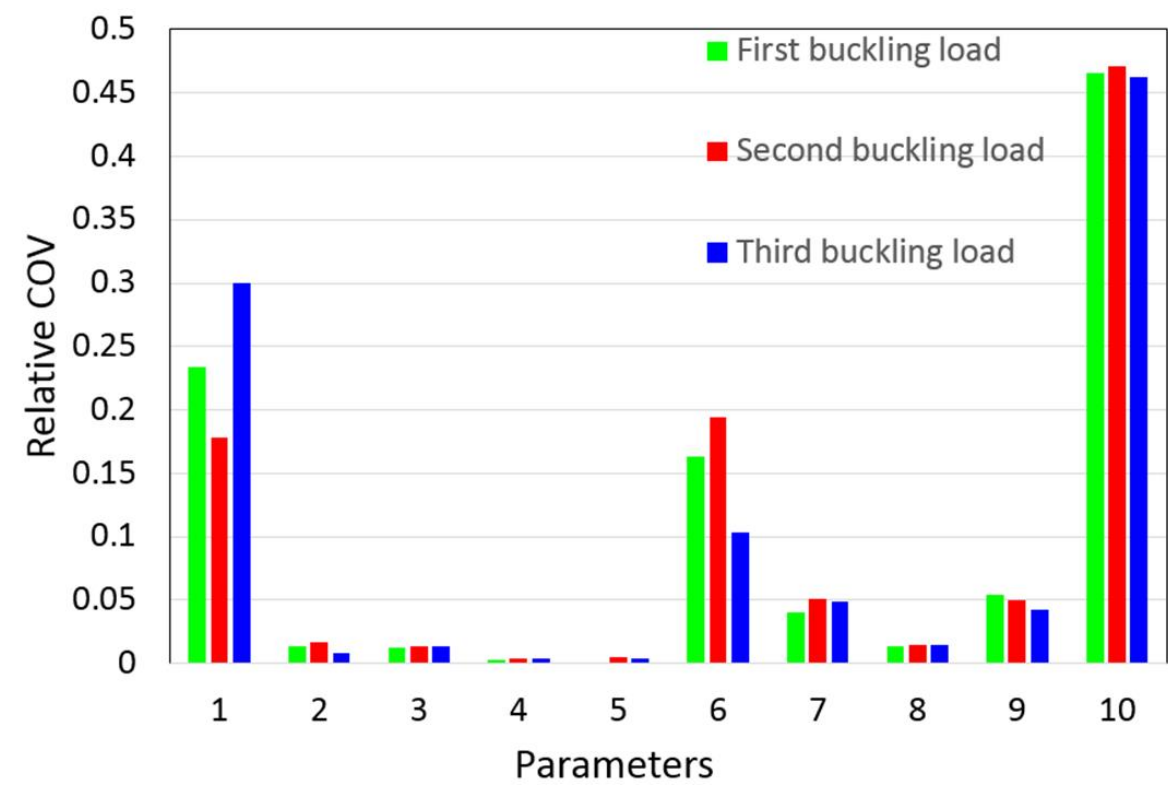

1- Longitudinal elastic modulus of fibre

2- Transverse elastic modulus of fibre

3- Poisson's ratio of fibre

4- Shear modulus of fibre

5- Mass density of fibre

6- elastic modulus of matrix

7- Shear modulus of matrix

8- Poisson's ratio of matix

9- Mass density of matrix

10- Volume fraction

(b)

Fig. 28 (a) Relative coefficient of variation (RCOV) for the buckling loads considering macro-mechanical material properties (b) Relative coefficient of variation (RCOV) for the buckling loads considering micromechanical material properties

the relative sensitivity of various stochastic input parameters (source-uncertainty) to the buckling loads in the macro and micro mechanical analyses. For stochasticity in macro-mechanical material properties, it is observed that longitudinal Young's modulus and transverse Young's modulus are most sensitive to the first and second buckling load, while the mass density and out-of-plane shear moduli are the least sensitive parameters. The Poisson's ratio is found to be most sensitive in case of the third buckling load. 


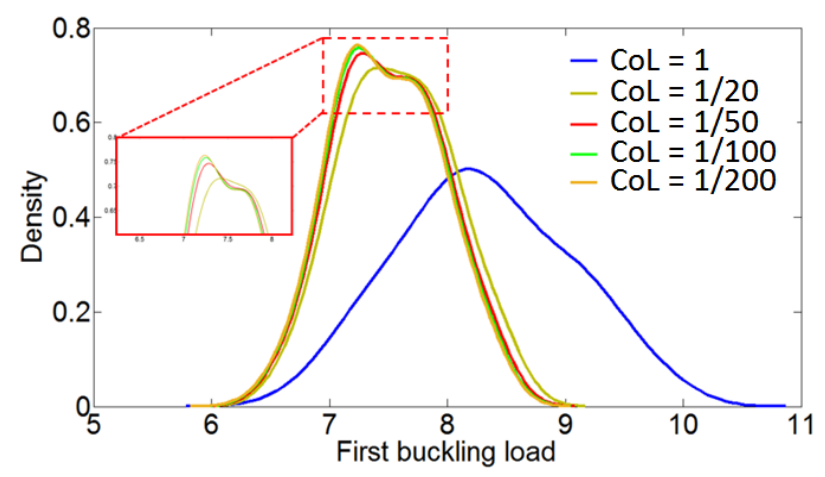

(a)

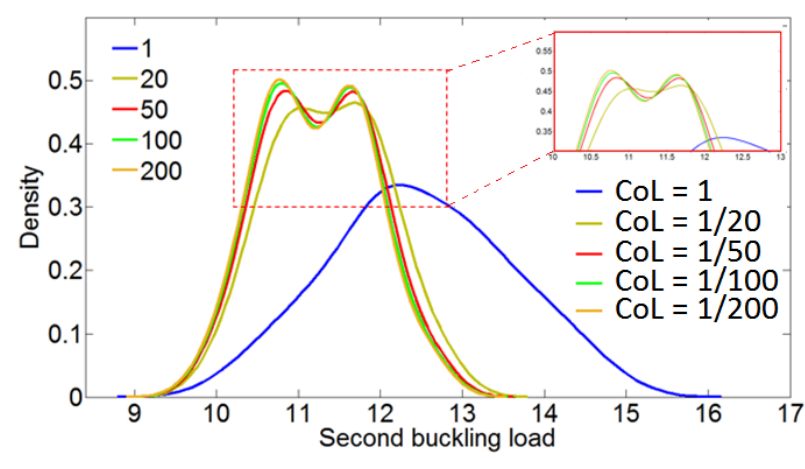

(b)

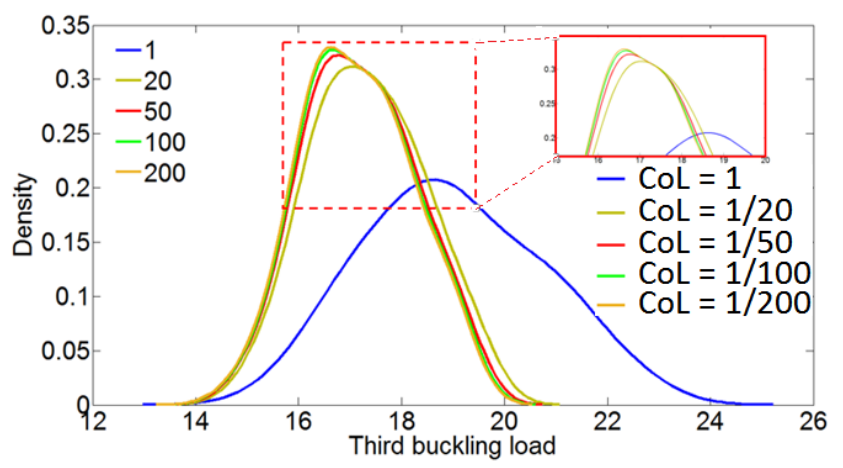

(c)

Fig. 29 Effect of correlation length $(\mathrm{CoL})$ on the probability distribution of the first three buckling loads of a composite laminate

For the micro-mechanical analysis, it is noticed that the most sensitive properties are volume fraction, longitudinal Young's modulus of fibre, Young's modulus of matrix, and shear modulus of matrix (in a decreasing order) while the sensitivity of the other parameters are negligible.

The probabilistic distribution of the buckling loads depend on correlation length of the random field considered for the macro and micromechanical properties. The spatial distribution and probabilistic descriptions of the material properties are discussed in the preceding section (refer to figure 16 and 17). The effect of correlation length on the probability distribution of the first three buckling loads of the composite laminate is presented in figure 29 , wherein a clear difference can be noticed between the lower and higher values of correlation lengths. The figure shows that the mean and response bound decrease in case of the random field based modelling of source-uncertainty compared to the random variable based modelling. The effect of stochasticity in micro and macro mechanical attributes is studied on the buckling mode shapes considering two different boundary conditions (SSSS and CCCC). The results are presented 


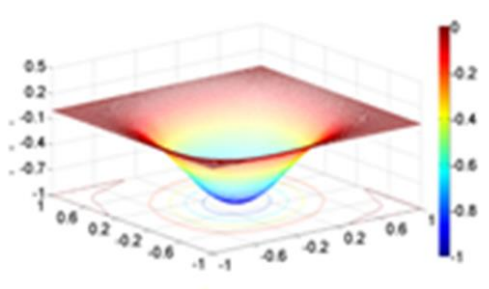

(a)

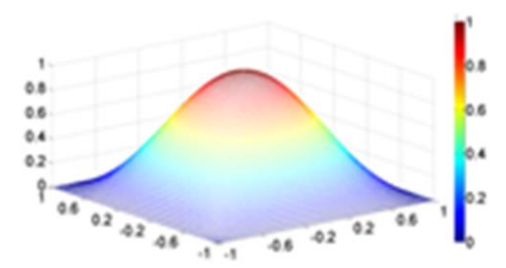

(d)

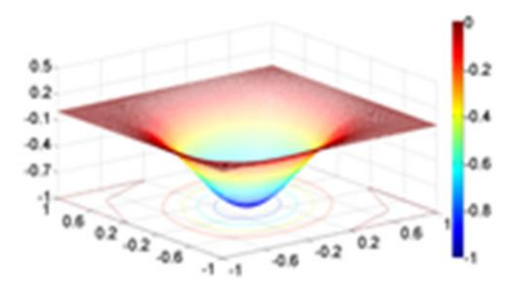

(g)

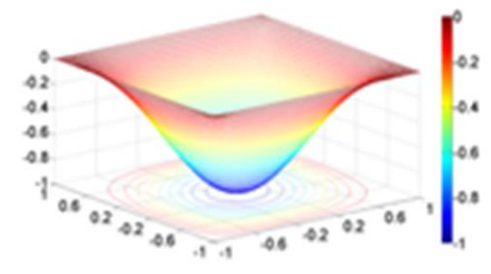

(j)

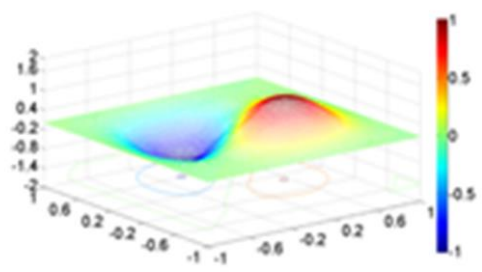

(b)

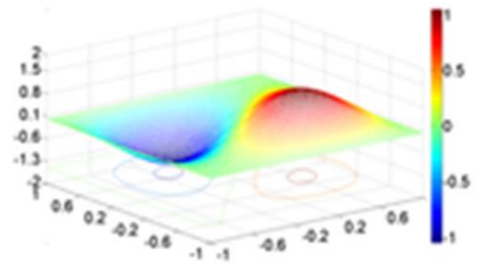

(e)

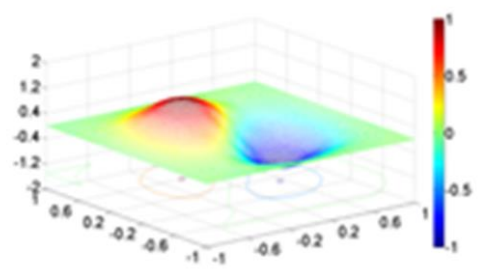

(h)

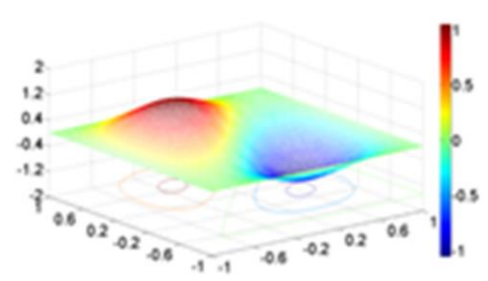

(k)

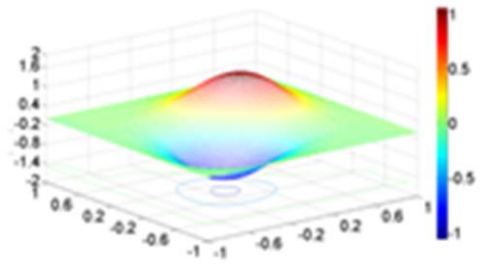

(c)

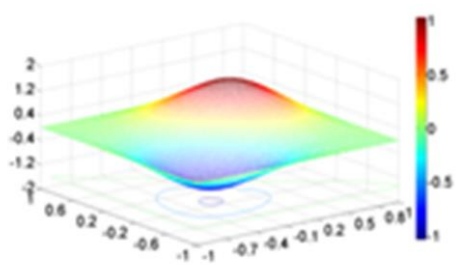

(f)

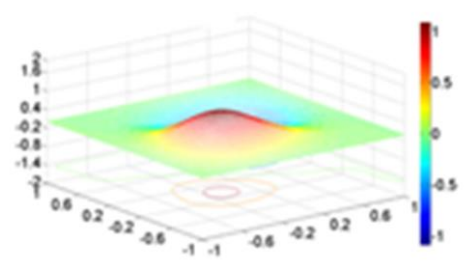

(i)

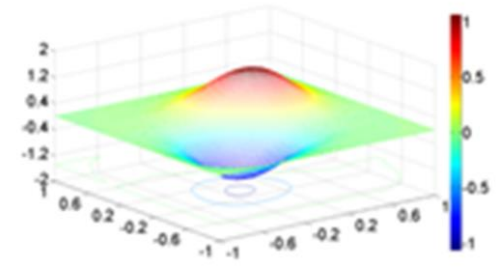

(1)

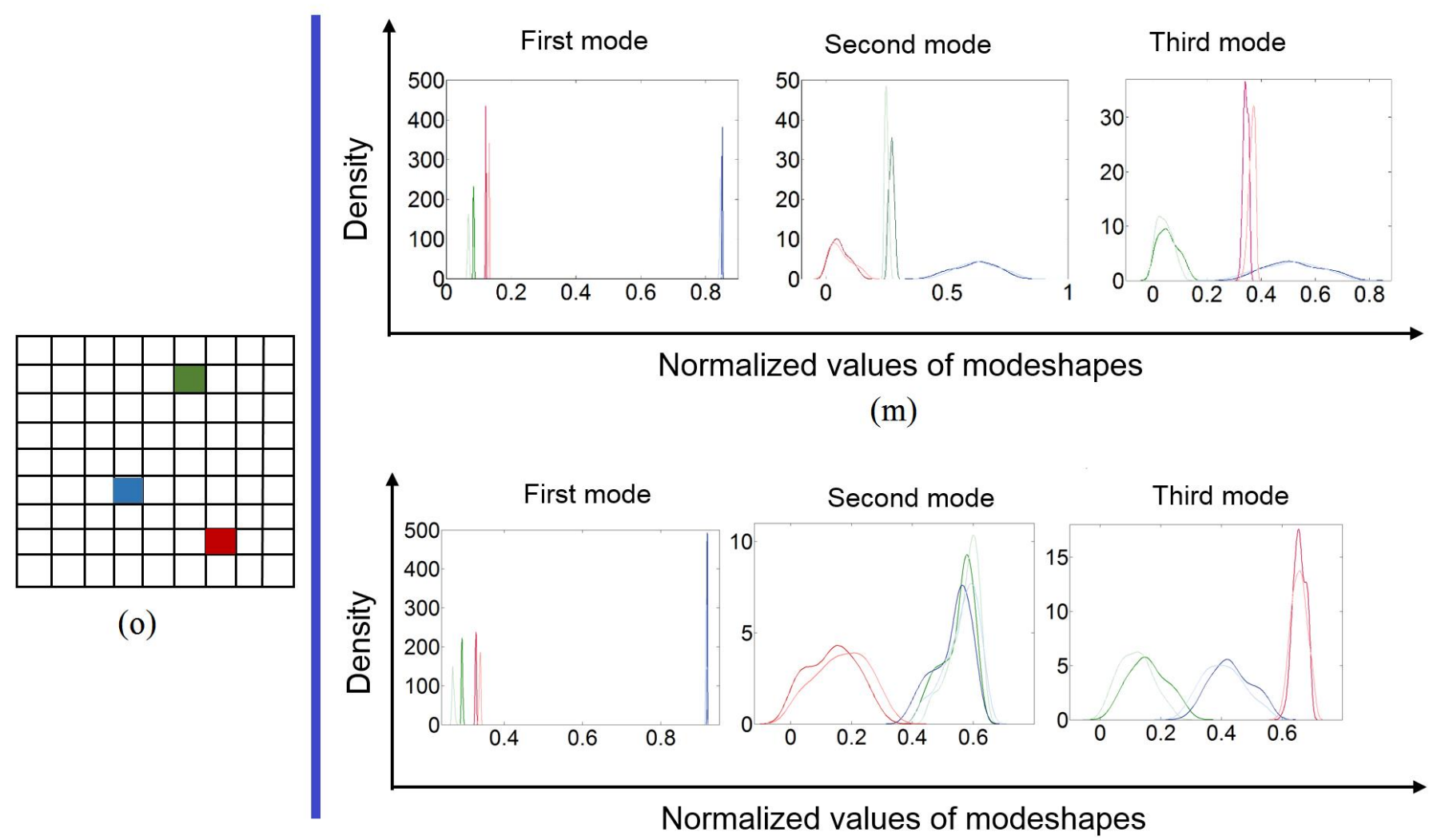

(n)

Fig. 30 Stochastic modeshapes and representative probability distribution of normalized eigenvectors 
in figure 30 for first three modes of vibration. Stochastic mode shapes for first three modes of buckling considering a single random realization are presented in case of: a clamped (CCCC) composite plate with stochasticity in the macromechanical $\left(g_{\text {macro }}^{C}\right)$ properties (refer to figure $30(\mathrm{a}-\mathrm{c})$ ), a simply supported (SSSS) composite plate with stochasticity in the macromechanical $\left(g_{\text {macro }}^{C}\right)$ properties (refer to figure $30(\mathrm{~d}-\mathrm{f}))$, a clamped $(\mathrm{CCCC})$ composite plate with stochasticity in the micromechanical $\left(g_{\text {micro }}^{C}\right.$ )properties (refer to figure 30(g-i)) and a clamped (CCCC) composite plate with stochasticity in the micromechanical $\left(g_{\text {micro }}^{C}\right.$ ) properties (refer to figure $30(j-1)$ ). From the mode shapes presented in figure 30(a - 1), it can be observed that the basic global pattern of the stochastic mode shapes remains similar to the deterministic case. However, the value of normalized eigenvectors becomes stochastic in nature for each of the elements in the composite plate. Probability distribution of the normalized eigenvectors of first three buckling modes for the elements indicated in figure 30(o) are shown considering a clamped (CCCC) boundary condition (refer to figure 30(m)) and a simply supported (SSSS) boundary condition (refer to figure 30(n)). The results for micro and macro mechanical analyses are shown using lighter and darker shades of respective colours indicated in figure $30(\mathrm{o})$. It can be noticed that the probability density function plots depend significantly on the type of analysis (micro and macro mechanical) and location of the element under consideration.

\section{Summary and perspective}

This paper presents an efiicient stochastic bottom-up framework for analyzing the dynamics and stabilty of composite laminates with spatially varying correlated system properties (refer to figure 31 ). A novel idea of SRVE is proposed in the context of two dimensional plate-like structures to incorporate the spatially varying material properties in conjunction with FE analysis. It can be noted here that the conventional methods of representative volume element (RVE) based analyses and other available analytical solutions can not consider the effect of spatial randomness of system properties. In the proposed SRVE based approach, various other spatially varying system properties (such as varying intensity of matrix cracking, varying fibre properties, fibre breakage etc.) can also be easily accounted in future. However, a stochastic simulation involving finite element analysis becomes exorbitantly 
computationally expensive. To mitigate this lacuna, a surrogate based approach (HDMR coupled with DMORPH), which has been proved to be suitable for modelling correlated input parameters (Li and Rabitz (2012)), is adopted to obtain computational efficiency (without compromising the accuracy of results) in the present analysis.

In the stochastic micromechanical characterization of composite laminates, there exists three distinct stages of the analysis: uncertainty modelling at the input level, propagation of uncertainty to the global level and quantification of the global responses such as dynamics and stability characteristics. In most of studies concerning the uncertainty quantification of composite structures, due to simplicity of the approach in implementation, a random variable based strategy is found to be adopted for modelling the material properties, wherein the input material properties are considered to be constant along the two dimensional space (as discussed in the introduction section) for a particular realization of Monte Carlo simulation. However, in reality, the material properties are not constant spatially in a plate-like structure; rather they are spatially varying. Moreover, a correlation often exists in the spatial distribution of the material properties. In the present study, we have adopted a practically relavant random field based uncertainty modelling strategy with correlated input parameter distribution. Two separate analyses have been performed considering the source-uncertainty in micro and macro mechanical properties to present a comparative perspective by higlighting the cascading effect in stochasticity. After modelling the uncertainty in a practically relavant way, the effect of uncertainty needs to be propagated towards the global responses from elementary input level. The propagation of uncertainty is carried out throgh a stochastic computational model of the structure based on Monte Carlo simulation. In general for complex composite laminated structures with spatially varying system properties, the performance functions are not normally available as explicit functions of the random input variables. Thus the performance functions or responses (such as natural frequencies and buckling loads) of the structure can only be computed numerically at the end of an intensive structural analysis procedure (such as the FE method), which is often exorbitantly time-consuming and computationally expensive. The surrogate based uncertainty propagation strategy, as adopted in this study, can develop a representative and predictive mathematical/ statistical metamodel relating the natural frequencies and buckling loads to a number of 


\section{Stochastic micromechanical}

analysis

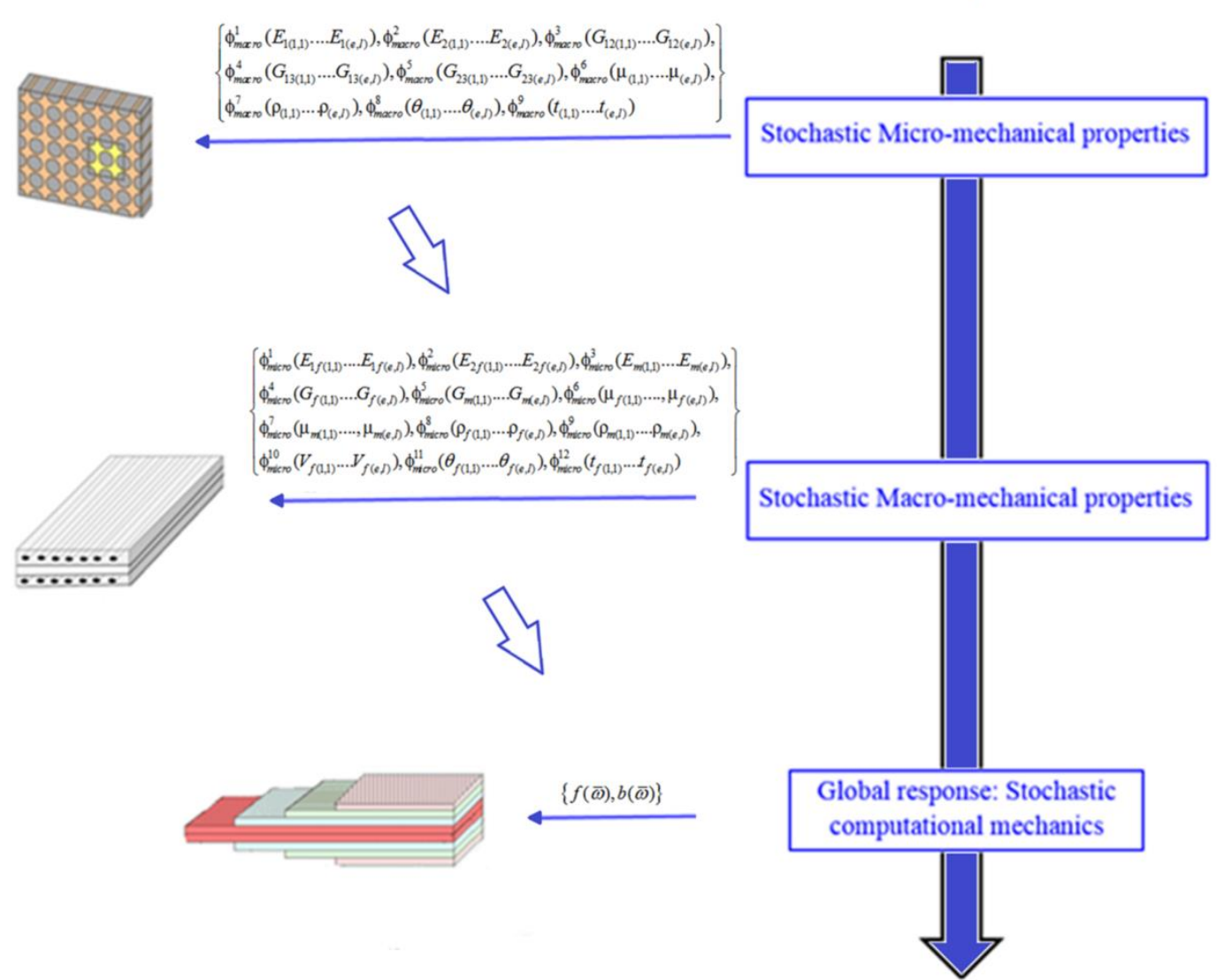

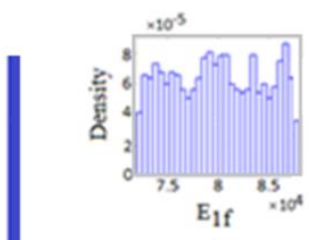

(a)

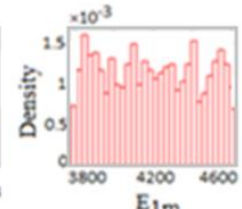

(b)

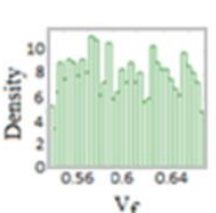

(c)

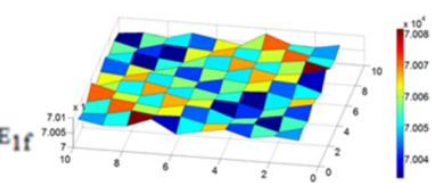

(d)

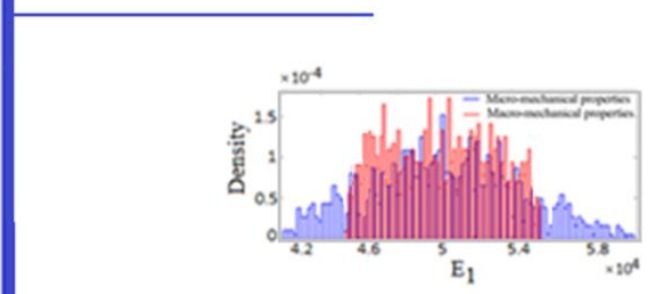

(e)

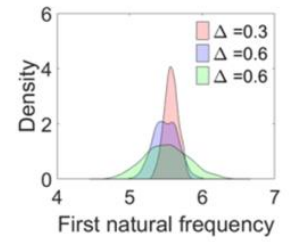

(g)

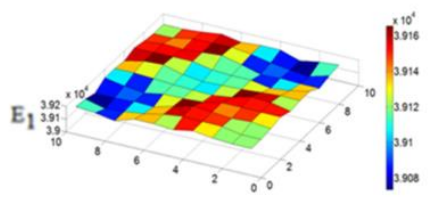

(f)

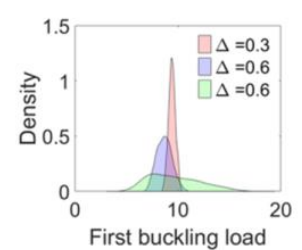

(h)

Fig. 31 (a - c) Typical distribution of the representative micromechanical properties concerning the longitudinal Young's modulus (d) Spatial variation of a representative micromechanical property corresponding to a random realization (e) Typical distribution of the representative macromechanical properties concerning the longitudinal Young's modulus for macromechanical (in red colour) and micromechanical (in blue colour) analyses (f) Spatial variation of a representative macromechanical property corresponding to a random realization $(\mathrm{g}-\mathrm{h})$ Representative results for the probability distributions of first natural frequency and first buckling load considering micro mechanical analysis for different degree of stochasticity (Here $f(\bar{\omega})$ and $b(\bar{\omega})$ denote the stochastic natural frequencies and stochastic buckling loads respectively) 
stochastic input variables. Thereafter the metamodels (response surface) are used to compute the dynamic and stability characteristics corresponding to a given set of input variables, instead of having to simulate repeatedly the time-consuming FE analysis. The response surface here represents the results (or outputs) of the structural analyses encompassing (in theory) every prospective combination of the stochastic input variables. Hence, thousands of combinations of the stochastic input variables can be created and a pseudo analysis (efficient, yet accurate) for each variable set can be performed by adopting the corresponding surrogate model. The final step in the stochastic analysis is uncertainty quantification in the output responses, which is effectively carried out by deriving the probabilistic distributions and the statistical moments.

In case of the micro-mechanical analysis, the source-uncertainty is accounted in the micromechanical material properties (spatially varying with correlated attributes) of composites. On the basis of the SRVE approach, the equivalent macro-scale material properties are computed by considering the stochasticity in micro-mechanical properties as shown in figure 31 . Thereby, the equivalent macro-scale material attributes are fed into the FE code to compute the global responses (like natural frequencies and buckling loads) of the composite plate. In the present paper, as we only deal with the spatial variation of material properties, there is no need for a finite element model at the micro-level to obtain the macromechanical material properties, rather exploitation of the Halpin-Tsai principle is the most efficient way. However, if the problem involves other spatially varying micromechanical structural features (such as damage), then one will need to carry out a finite element modelling at the micro level to obtain the effective macromechanical properties. In the present micromechanical analysis, stochasticity is essentially accounted at the micro-scale level first, and then the effect is propagated to the macro-scale level to characterize the global responses of the structural system. It is common in scientific literature to refer this genre of analysis for composite materials and structures considering micro-mechanical properties (i.e. involving multiple length-scales) based on representative volume element (RVE) as multi-scale analysis (May et al. (2014)). As stochatic material and structural parameters are considered in the present analysis leading to probabilistic characterization of the structural responses, the study is referred as probabilistic micromechanical analysis in this paper. It can be noted in this context that various other micro- 
mechanical attributes (for example micro-scale spatially random damage) can be considered using the proposed framework of SRVE. Thus the concept of SRVE can be regarded as a generalization of the concept of conventional RVE in the stochastic regime involving randomly inhomogeneous systems.

The results in this article capture the influence of inevitable source-uncertainty across the lengthscales in a practically relavant manner. In-depth results are presented for the dynamic and stabilty analysis considering various structural configurations and stochasticity models. It is noted that the stochastic system, in two different limiting cases of the correlation length, tends to become a randomly homogeneous syestem (for very high value of correlation length) and randomly inhomogeneous system with uncorrelated properties (for very low value of correlation length). A general proclivity of increasing response bound and standard deviation for the output quantities are observed with higher degree of stochasticity in the design parameters. For a particular degree of stochasticity, it is noticed that the standard deviation is higher in case of micromechanical analysis than that of the macromechanical analysis, affirming that the probabilistic descriptions of the response quantities substantially differ from each other on the basis of the adopted type of analysis. A micromechanical analysis, which is more accurate for considering the source uncertainty at a more elementary level, shows higher degree of variability in the global responses due to the cascading effect in stochasticity. The stochastic response bounds are found to be up to more than $60 \%$ higher in case of the micromechanical analyses compared to the respective macromechanical analyses (refer to figures 11 and 24). case of stochastic stability analysis, the buckling loads are found to have a response bound (depending on the degree of stochasticity) with respect to the corresponding deterministic values (for instance refer to figure 25). Thus there exists a possibility of overlap in the stochastic responses of the buckling loads corresponding to different modes of buckling resulting in a non-unique critical buckling mode with the minimum value of buckling load. For this reason, it is noted to be essential to consider higher buckling modes in case of considering stochasticity in the system parameters.

For stochasticity in macro-mechanical attributes, it is observed that mass density, longitudinal Young's modulus and transverse Young's modulus (in decreasing order of sensitivity) are most sensitive to the first three modes of free vibration, while for the micro-mechanical analysis, it is observed that the 
most sensitive atributes in decreasing order of sensitivity are mass density of fibre, longitudinal Young's modulus of fibre and volume fraction. In case of the buckling analysis considering stochasticity in macromechanical attributes, it is observed that longitudinal Young's modulus, transverse Young's modulus and Poisson's ratio are most sensitive parameters, while for the micro-mechanical analysis, it is noted that the most sensitive parameters are volume fraction, longitudinal Young's modulus of fibre, Young's modulus of matrix and shear modulus of matrix. The parameters with negligible sensitivity to different response quantities are also identified. Two different forms of analyses, as carried out here accounting the macro and micro mechanical material properties, render an in-depth understanding regarding the relative influence of various stochastic input parameters (source-uncertainty). The results of sensitivity analyses will allow the manufacturers, designers and maintenace personnel to ensure an effective quality control for the structural system.

\section{Conclusion}

A bottom-up micromechanical probabilistic analysis is presented in this article for quantifying the effect of source-uncertainty in the dynamics and stability behaviour of composite plates considering spatially random system attributes. For this purpose, a physically relevant random field based modelling approach with correlated material properties is adopted based on the Karhunen-Loève expansion. A concept of stochastic representative volume element (SRVE) is adopted in the context of two dimensional plate-like structures to consider the spatially varying randomly inhomogeneous form of uncertainty. The probability distributions of the response quantities (such as natural frequencies and buckling loads) due to the source-uncertainty in micro-mechanical properties are compared with that arising from a same degree of stochasticity in the macro-mechanical properties to portray the cascading effect in the stochastic analysis involving different length-scales. The stochastic response bounds are found to be up to more than $60 \%$ higher in case of the micromechanical analyses compared to the respective macromechanical analyses. Effect of the source-uncertainty is investigated on the modeshapes of composite plates. It is noted that the higher buckling modes may assume a critical role in stochastic analysis of composite plates. To understand the relative influence of different input parameters, sensitivity of the micro and macro mechanical material parameters are analysed for the dynamic and stability characteristics of the composite 
plate. For achieving computational efficiency, a coupled HDMR and DMORPH based stochastic analysis algorithm is developed in conjunction with the probabilistic finite element model, wherein it is noted that the required number of original FE simulations can be remarkably reduced without compromising accuracy of results.

It is found that the stochasticity/ source-uncertainty have significant influence on the dynamic and stability behaviour of composite structures. Thus it is imperative to consider the effect of these uncertainties in subsequent analyses/ design process to ensure the desired robust and sustainable system performance. Novelty of this paper includes the consideration of correlated spatial variation of material properties (micro/ macro) following the SRVE approach coupled with the HDMR algorithm in context to composite laminates. The idea of SRVE is quite generic in nature; this concept can be extended to other forms of structures and various stochastic systems with spatial randomness in two/ three dimensions. Moreover, the computationally efficient coupled HDMR and DMORPH based approach for uncertainty quantification in laminated composites can be extended further to other structures and mechanical systems to characterize various output responses in future.

\section{Acknowledgements}

SN and SS are grateful for the support provided through the Lloyd's Register Foundation Centre. The Foundation helps to protect life and property by supporting engineering-related education, public engagement and the application of research.

\section{APPENDIX}

In present article, a laminated composite plate with thickness $h$, length $L$ and width $b$ is analysed as shown in figure 2 and 3. According to the first-order shear deformation theory, the displacement field can be described as

$$
\begin{aligned}
& u(x, y, z)[\bar{\omega}]=u^{0}(x, y)[\bar{\omega}]-z \theta_{x}(x, y)[\bar{\omega}] \\
& v(x, y, z)[\bar{\omega}]=v^{0}(x, y)[\bar{\omega}]-z \theta_{y}(x, y)[\bar{\omega}] \\
& w(x, y, z)[\bar{\omega}]=w^{0}(x, y)[\bar{\omega}]=w(x, y)[\bar{\omega}]
\end{aligned}
$$


where, $u^{0}(\bar{\omega}), v^{0}(\bar{\omega})$, and $w^{0}(\bar{\omega})$ denote displacements of the reference plane and $\theta_{x}(\bar{\omega})$ and $\theta_{y}(\bar{\omega})$ represent the rotations of cross section relative to $\mathrm{x}$ and $\mathrm{y}$ axes, respectively. Each fibre of laminae can have an arbitrary orientation angle ' $\theta$ ' with respect to the x-axis. Here $\bar{\omega}$ is used to denote the stochastic character of the system parameters and matrices. The corresponding strain field is given by

$$
\begin{gathered}
\varepsilon_{x x}=\frac{\partial u^{0}}{\partial x}+z \frac{\partial \theta_{x}}{\partial x}, \varepsilon_{y y}=\frac{\partial v^{0}}{\partial y}+z \frac{\partial \theta_{y}}{\partial y}, \varepsilon_{z z}=0 \\
\gamma_{x y}=\frac{\partial u^{0}}{\partial y}+\frac{\partial v^{0}}{\partial x}+z\left(\frac{\partial \theta_{x}}{\partial y}+\frac{\partial \theta_{y}}{\partial x}\right), \gamma_{x z}=\frac{\partial w^{0}}{\partial x}+\theta_{x}, \gamma_{y z}=\frac{\partial w^{0}}{\partial y}+\theta_{y}
\end{gathered}
$$

The plane of elastic symmetry is given by

$$
\left[\begin{array}{c}
\sigma_{x} \\
\sigma_{y} \\
\tau_{x y} \\
\tau_{y z} \\
\tau_{x z}
\end{array}\right]=\left[\begin{array}{lllll}
Q_{11} & Q_{12} & Q_{16} & 0 & 0 \\
Q_{12} & Q_{22} & Q_{26} & 0 & 0 \\
Q_{16} & Q_{26} & Q_{66} & 0 & 0 \\
0 & 0 & 0 & Q_{44} & Q_{45} \\
0 & 0 & 0 & Q_{45} & Q_{55}
\end{array}\right]
$$

where $Q_{i j}(i, j=1,2,6)$ are the plane stress reduced stiffness components and $Q_{i j}(i, j=4,5)$ are the transverse shear stiffness components of the layer material. The force and moment resultants per unit length are given by (Bertholet (1999))

$$
\begin{gathered}
\left(N_{x}, N_{y}, N_{x y}\right)[\bar{\omega}]=\int_{\frac{-h}{2}}^{\frac{h}{2}}\left(\sigma_{x}, \sigma_{y}, \tau_{x y}\right)[\bar{\omega}] d z \\
\left(Q_{x}, Q_{y}\right)[\bar{\omega}]=\int_{\frac{-h}{2}}^{\frac{h}{2}}\left(\tau_{x z}, \tau_{y z}\right)[\bar{\omega}] d z, \\
\left(M_{x}, M_{y}, M_{x y}\right)[\bar{\omega}]=\int_{\frac{-h}{2}}^{\frac{h}{2}}\left(\sigma_{x}, \sigma_{y}, \tau_{x y}\right)[\bar{\omega}] z d z
\end{gathered}
$$

where $N_{x}, N_{y}$ and $N_{x y}$ are the in plane force resultants, $Q_{x}$ and $Q_{y}$ are the transverse force resultants, $M_{x}$ and $M_{y}$ are bending moment resultants, and $M_{x y}$ is the twisting moment resultants. The constitutive equation of laminates according to the shear deformation theory of first order associates the resultants and moments as follows: 


$$
\left[\begin{array}{l}
N_{x} \\
N_{y} \\
N_{x y} \\
M_{x} \\
M_{y} \\
M_{x y} \\
Q_{y} \\
Q_{z}
\end{array}\right]=\left[\begin{array}{llllllll}
A_{11} & A_{12} & A_{16} & B_{11} & B_{12} & B_{16} & 0 & 0 \\
A_{12} & A_{22} & A_{26} & B_{12} & B_{22} & B_{26} & 0 & 0 \\
A_{16} & A_{26} & A_{66} & B_{16} & B_{26} & B_{66} & 0 & 0 \\
B_{11} & B_{12} & B_{16} & D_{11} & D_{12} & D_{16} & 0 & 0 \\
B_{12} & B_{22} & B_{26} & D_{12} & D_{22} & D_{26} & 0 & 0 \\
B_{16} & B_{26} & B_{66} & D_{16} & D_{26} & D_{66} & 0 & 0 \\
0 & 0 & 0 & 0 & 0 & 0 & S_{44} & S_{45} \\
0 & 0 & 0 & 0 & 0 & 0 & S_{45} & S_{55}
\end{array}\right]\left[\begin{array}{l}
\partial u^{0} / \partial x \\
\partial v^{0} / \partial y \\
\partial u^{0} / \partial y+\partial v^{0} / \partial x \\
\partial \theta_{x} / \partial x \\
\partial \theta_{y} / \partial y \\
\partial \theta_{x} / \partial y+\partial \theta_{y} / \partial y \\
\partial w^{0} / \partial y+\theta_{y} \\
\partial w^{0} / \partial x+\theta_{x}
\end{array}\right]
$$

The $A_{i j}, B_{i j}$ and $D_{i j}$ matrices are given by

$$
\begin{gathered}
{\left[A_{i j}(\bar{\omega}), \quad B_{i j}(\bar{\omega}), \quad D_{i j}(\bar{\omega})\right]=\sum_{k=1}^{n} \int_{z_{k-1}}^{z_{k}}\left[Q_{i j}(\bar{\omega})\right]_{k}\left[1, \quad z, z^{2}\right] d z \quad i, j=1,2,6} \\
{\left[S_{i j}(\bar{\omega})\right]=\sum_{k=1}^{n} \int_{z_{k-1}}^{z_{k}} \alpha_{s}\left[Q_{i j}(\bar{\omega})\right]_{k} d z \quad i, j=4,5}
\end{gathered}
$$

where $\left[Q_{i j}\right]_{k}$ are stiffness coefficients of the $k$-th layer and $\alpha_{s}$ denotes the shear correction factor $(=5 / 6)$.

To study the dynamic behaviour of composite laminates, Hamilton's principle (Meirovitch (1992)) can be used. The principle for Lagrangian is defined as

$$
L_{f}(\bar{\omega})=T(\bar{\omega})-U(\bar{\omega})-W(\bar{\omega})
$$

where $T(\bar{\omega}), U(\bar{\omega})$ and $W(\bar{\omega})$ denote the total kinetic energy, total strain energy and total potential of the applied load, respectively. The Hamilton's principle for a non-conservative system is

$$
\delta H(\bar{\omega})=\int_{t_{i}}^{t_{f}}[\delta T(\bar{\omega})-\delta U(\bar{\omega})-\delta W(\bar{\omega})] d t=0
$$

The energy functional of Hamilton's principle (Lagrangian $(\mathrm{L}(\bar{\omega})))$ includes kinetic energy $(\mathrm{T}(\bar{\omega}))$ and potential strain energy $(\mathrm{U}(\bar{\omega}))$ for an elastic body. The expression of the kinetic energy for an element is

$$
T(\bar{\omega})=\frac{1}{2}\left\{\dot{\delta}_{e}(\bar{\omega})\right\}^{T}\left[M_{e}(\bar{\omega})\right]\left\{\dot{\delta}_{e}(\bar{\omega})\right\}
$$

where $\left[M_{e}\right]$ represents the element-level mass matrix. The parameter $\left\{\delta_{e}\right\}$ is the elemental displacement vector. The potential strain energy of an element can be expressed as, 


$$
U(\bar{\omega})=U_{1}(\bar{\omega})+U_{2}(\bar{\omega})=\frac{1}{2}\left\{\delta_{e}(\bar{\omega})\right\}^{T}\left[K_{e}(\bar{\omega})\right]\left\{\delta_{e}(\bar{\omega})\right\}+\frac{1}{2}\left\{\delta_{e}(\bar{\omega})\right\}^{T}\left[K_{\sigma e}(\bar{\omega})\right]\left\{\delta_{e}(\bar{\omega})\right\}
$$

where $\left[K_{\sigma e}(\bar{\omega})\right]$ and $\left[K_{e}(\bar{\omega})\right]$ are geometric stiffness matrix and elastic stiffness matrix respectively. The Langrange's equation of motion can be expressed as

$$
\frac{d}{d t}\left[\frac{\partial L_{f}(\bar{\omega})}{\partial \delta_{e}}\right]-\left[\frac{\partial L_{f}(\bar{\omega})}{\partial \delta_{e}}\right]=\left\{F_{e}(\bar{\omega})\right\}
$$

where $\left\{F_{e}(\bar{\omega})\right\}$ denote the external elemental force vector and $L_{f}(\bar{\omega})$ represents the Lagrangian function. By replacing the value of $L_{f}$, stochastic dynamic equilibrium expression for each of the elements is obtained as

$$
[M(\bar{\omega})]\left\{\ddot{\delta}_{e}(\bar{\omega})\right\}+\left(\left[K_{e}(\bar{\omega})\right]+\left[K_{\sigma e}(\bar{\omega})\right]\left\{\delta_{e}(\bar{\omega})\right\}\right)=\left\{F_{e}(\bar{\omega})\right\}
$$

The governing equation for stochastic free vibration analysis without damping is expressed as

$$
[M(\bar{\omega})]\{\ddot{\delta}(\bar{\omega})\}+[K(\bar{\omega})]\{\delta(\bar{\omega})\}=0
$$

where $[K(\bar{\omega})]=\left[K_{e}(\bar{\omega})\right]+\left[K_{\sigma e}(\bar{\omega})\right]$. In the finite element formulation of this study, an eight noded element is considered, wherein each node has five degrees of freedom (two rotations and three translations). The natural frequencies $\left(\omega_{k}\right)$ and mode shapes $\left(S_{k}^{f}\right)$ of the composite plate are obtained by solving an eigenvalue problem based on QR iteration algorithm (Bathe (1990), Rayleigh (1945))

$$
[K(\bar{\omega})] S_{k}^{f}(\bar{\omega})=\left[\omega_{k}(\bar{\omega})\right]^{2}[M(\bar{\omega})] S_{k}^{f}(\bar{\omega})
$$

where $k=1,2,3, \ldots, n$. The superscript $f$ is used to denote frequency analysis. Here the orthogonality relationship is satisfied as

$$
\left[S_{i}^{f}(\bar{\omega})\right]^{T}[M(\bar{\omega})] S_{k}^{f}(\bar{\omega})=\bar{\delta}_{i k} \text { and }\left[S_{i}^{f}(\bar{\omega})\right]^{T}[K(\bar{\omega})] S_{k}^{f}(\bar{\omega})=\left[\omega_{k}(\bar{\omega})\right]^{2} \bar{\delta}_{i k}
$$

where $i, k=1,2,3, \ldots . n$ and the Kronecker delta functions $\bar{\delta}_{i k}=0$ for $i \neq k ; \bar{\delta}_{i k}=1$ for $i=k$. The problem of stability analysis is solved through another eigenvalue problem obtained by minimizing the total potential energy (refer to equation 10) as:

$$
\left[K_{e}(\bar{\omega})\right] S_{k}^{b}=\lambda^{b}(\bar{\omega})\left[K_{\sigma e}(\bar{\omega})\right] S_{k}^{b}(\bar{\omega})
$$

where $\lambda^{b}(\bar{\omega})$ is the stochastic buckling load factor and $S_{k}^{b}$ gives the buckling modeshapes. The superscript $b$ is used to denote buckling analysis. 


\section{References}

Agarwal H, Renaud J E, Preston E L, Padmanabhan D, Uncertainty quantification using evidence theory in multidisciplinary design optimization, Reliability Engineering \& System Safety, 85 281-94, 2004.

Bathe K J, Finite Element Procedures in Engineering Analysis, PHI, New Delhi, India, 1990.

Bertholet J M, Composite materials - Mechanical behavior and structural analysis, Springer Verlag, New York, 1999.

Biswal, M., Sahu, S. K., \& Asha, A. V., Vibration of composite cylindrical shallow shells subjected to hygrothermal loading-experimental and numerical results. Composites Part B: Engineering, 98, 108119,2016

Chakrabarti A, Chalak H D, Iqbal M A and Sheikh A H, A new FE model based on higher order zigzag theory for the analysis of laminated sandwich beam with soft core. Composite Structures, 93(2) 271$279,2011$.

Chakrabarti A, Sheikh A H, Griffith M, Oehlers D J, Dynamic Response of composite beams with partial shear interactions using a higher order beam theory, Journal of Structural Engineering, 139 (1) 47-56, 2013.

Charmpis D C, Schuëller G I, Pellissetti M F, The need for linking micromechanics of materials with stochastic finite elements: a challenge for materials science, Computational Materials Science, $4127-$ 37, 2007.

Deodatis G, Shinozuka M, The weighted integral method, ii: response variability and reliability, Journal of Engineering Mechanics-ASCE, 117 (8) 1865-1877, 1991.

Deodatis G, The weighted integral method, i: stochastic stiffness matrix, Journal of Engineering Mechanics-ASCE, 117 (8) 1851-1864, 1991.

Dey P, Haldar S, Sengupta D, Sheikh A H, An efficient plate element for the vibration of composite plates, Applied Mathematical Modelling, 40 (9-10) 5589-5604, 2016d.

Dey S, Mukhopadhyay T, Adhikari S, Stochastic free vibration analysis of angle-ply composite plates-a RS-HDMR approach, Composite Structures, 122 526-536, $2015 \mathrm{c}$.

Dey S, Mukhopadhyay T, Khodaparast H H, Adhikari S, Fuzzy uncertainty propagation in composites using Gram-Schmidt polynomial chaos expansion, Applied Mathematical Modelling, 40 (7-8) 44124428, $2016 b$.

Dey S, Mukhopadhyay T, Khodaparast H H, Kerfriden P, Adhikari S, Rotational and ply-level uncertainty in response of composite shallow conical shells, Composite Structures, 131 594-605, 2015 b.

Dey S, Mukhopadhyay T, Naskar S, Dey T K, Chalak H D, Adhikari S (2019) Probabilistic characterization for dynamics and stability of laminated soft core sandwich plates, Journal of Sandwich Structures \& Materials, DOI: 10.1177/1099636217694229. 
Dey S, Mukhopadhyay T, Sahu S K, Li G, Rabitz H, Adhikari S, Thermal uncertainty quantification in frequency responses of laminated composite plates, Composites Part B: Engineering, 80 186-197, 2015a.

Dey S, Mukhopadhyay T, Spickenheuer A, Adhikari S, Heinrich G, Bottom up surrogate based approach for stochastic frequency response analysis of laminated composite plates, Composite Structures, 140 712-727, 2016c.

Dey S, Mukhopadhyay T, Spickenheuer A, Gohs U, Adhikari S, Uncertainty quantification in natural frequency of composite plates - An Artificial neural network based approach, Advanced Composites Letters, 25(2) 43-48, $2016 \mathrm{a}$.

Dey S., Mukhopadhyay T., Khodaparast H. H., Adhikari S. (2015d) Stochastic natural frequency of composite conical shells, Acta Mechanica, 226 (8) 2537-2553

Dey S., Mukhopadhyay T., Adhikari S. (2017) Metamodel based high-fidelity stochastic analysis of composite laminates: A concise review with critical comparative assessment, Composite Structures, 171 $227-250$

Dey S., Mukhopadhyay T., Adhikari S. (2018a) Uncertainty quantification in laminated composites: A meta-model based approach, CRC Press, Taylor \& Francis Group, ISBN 9781498784450

Dey S., Mukhopadhyay T., Khodaparast H. H., Adhikari S. (2016f) A response surface modelling approach for resonance driven reliability based optimization of composite shells, Periodica Polytechnica - Civil Engineering, 60 (1) 103-111

Dey S., Mukhopadhyay T., Sahu S. K., Adhikari S. (2016e) Effect of cutout on stochastic natural frequency of composite curved panels, Composites Part B: Engineering, 105, 188-202

Dey S., Mukhopadhyay T., Sahu S. K., Adhikari S. (2018b) Stochastic dynamic stability analysis of composite curved panels subjected to non-uniform partial edge loading, European Journal of Mechanics / A Solids, 67 108-122

Dey S., Naskar S., Mukhopadhyay T., Gohs U., Sriramula S., Adhikari S., Heinrich G. (2016g) Uncertain natural frequency analysis of composite plates including effect of noise - A polynomial neural network approach, Composite Structures, 143 130-142

Dey T.K., Mukhopadhyay T., Chakrabarti A., Sharma U.K.(2015e) Efficient lightweight design of FRP bridge deck, Proceedings of the Institution of Civil Engineers - Structures and Buildings, 168 (10) 697 707

Fang Chin, Springer George S, Design of composite laminates by a Monte Carlo method. Compos Mater, 27(7) 721-53, 1993.

Faure H, Good permutations for extreme discrepancy, J Number Theory, 42 47-56, 1992.

Galanti S, Jung A, Low-discrepancy sequences: Monte Carlo simulation of option prices. J Deriv 5 6383, 1997. 
Goyal V K, Kapania R K, Dynamic stability of uncertain laminated beams subjected to subtangential loads, Int J Solids Struct, 45(10) 2799-817, 2008.

Haciyev V. C., Sofiyev A. H., Kuruoglu, N., Free bending vibration analysis of thin bidirectionally exponentially graded orthotropic rectangular plates resting on two-parameter elastic foundations. Composite Structures, 184, 372-377, 2018.

Halton $\mathbf{J} \mathrm{H}$, On the efficiency of certain quasi-random sequences of points in evaluating multidimensional integrals, Numer Math, 2 84-90, 1960.

Huang S P, Quek S T, Phoon K K, Convergence study of the truncated karhunen-loeve expansion for simulation of stochastic processes, International Journal for Numerical Methods in Engineering, 52 1029-1043, 2001.

Jones R M, Mechanics of Composite structures, Taylor \& Francis, Philadelphia, PA, 1999.

Kapania R K, Goyal V K, Free vibration of unsymmetrically laminated beams having uncertain plyorientations, AIAA J, 40(11) 2336-44, 2002.

Karhunen K, ÃIJber lineare methoden in der wahrscheinlichkeitsrechnung, Annales Academiae Scientiarum Fennicae Ser., A137, 1947.

Karsh P K, Mukhopadhyay T, Dey S, Spatial vulnerability analysis for the first ply failure strength of composite laminates including effect of delamination, Composite Structures, 184 554-567, 2018 a.

Karsh P. K., Mukhopadhyay T., Dey S. (2018b) Stochastic dynamic analysis of twisted functionally graded plates, Composites Part B: Engineering, 147 259-278

Kazimierz S, Kirkner D J, Stochastic Modeling of Microstructures, Birkhäuser Basel, DOI: 10.1007/9781-4612-0121-2, 2001.

Klintworth J W, Stronge W J, Elasto-plastic yield limits and deformation laws for transversely crushed honeycombs, International Journal of Mechanical Sciences, 30 (3-4) 273- 292, 1988.

Kumari, P., \& Behera, S., Three-dimensional free vibration analysis of levy-type laminated plates using multi-term extended Kantorovich method. Composites Part B: Engineering, 116, 224-238, 2017

Lal A, Singh B N, Stochastic Free Vibration of Laminated Composite Plates in Thermal Environments, Journal of Thermoplastic Composite Materials, 23(1) 57-77, 2010.

Li C C, Der Kiureghian A, Optimal discretization of random fields, Journal of Engineering Mechanics, 119 (6) 1136-1154, 1993.

Li G, Hu J, Wang S W, Georgopoulos P G, Schoendorf J, Rabitz H, Random sampling-high dimensional model representation (RS-HDMR) and orthogonality of its different order component functions, J. Phys. Chem. A, 110 2474-2485, 2006.

Li G, Rabitz H, General formulation of HDMR component functions with independent and correlated variables, Journal of Mathematical Chemistry, 50(1) 99-130, 2012.

Li G, Wang S W, Rabitz H, Practical approaches to construct RS-HDMR component functions, J Phys Chem A, 106 8721-33, 2002a. 
Li G, Wang S W, Rabitz H, Wang S, Jaffe P, Global uncertainty assessments by high dimensional model representations, Chem Eng Sci, 57:4445-60, 2002b.

Liew K M, Huang Y Q, Bending and buckling of thick symmetric rectangular laminates using the moving least-squares differential quadrature method, International Journal of Mechanical Sciences, 45 95-114, 2003.

Liew K. M., Solving the vibration of thick symmetric laminates by reissner/mindlin plate theory and the p-ritz method, Journal of Sound and Vibration, 198(3) 343-360, 1996

Liu W, Belytschko T, Mani A, Probabilistic finite elements for nonlinear structural dynamics, Computer Methods in Applied Mechanics and Engineering, 56 (1) 61-81, 1986a.

Liu W, Belytschko T, Mani A, Random field finite elements, International Journal for Numerical Methods in Engineering, 23 (10) 1831-1845, 1986b.

Loève M, Probability Theory, Springer-Verlag, fouth Edition, 1977.

Maharshi K., Mukhopadhyay T., Roy B., Roy L., Dey S. (2018) Stochastic dynamic behaviour of hydrodynamic journal bearings including the effect of surface roughness, International Journal of Mechanical Sciences, 142-143 370-383

Mahata A., Mukhopadhyay T., Adhikari S. (2016) A polynomial chaos expansion based molecular dynamics study for probabilistic strength analysis of nano-twinned copper, Materials Research Express, 3036501

Manan A, Cooper J, Design of composite wings including uncertainties: a probabilistic approach, J Aircr, 46(2) 601-7, 2009.

Mandal A, Ray C, Haldar S, Free vibration analysis of laminated composite skew plates with cut-out, Archive of Applied Mechanics, 87 (9) 1511-1523, 2017.

Matthies H G, Brenner C E, Bucher C G, Guedes Soares C, Uncertainties in probabilistic numerical analysis of structures and solids-stochastic finite elements, Structural Safety, 19 (3) 283-336, 1997.

May M, Nossek M, Petrinic N, Hiermaier S, Thoma K, Adaptive multi-scale modeling of high velocity impact on composite panels, Composites: Part A, 58 56-64, 2014.

Meirovitch L, Dynamics and control of structures, NY: John Wiley \& Sons, 1992.

Metya S., Mukhopadhyay T., Adhikari S., Bhattacharya G. (2017) System Reliability Analysis of Soil Slopes with General Slip Surfaces Using Multivariate Adaptive Regression Splines, Computers and Geotechnics, 87 212-228

Mrabet E, Guedri M, Ichchou M N, Ghanmi S, Stochastic structural and reliability based optimization of tuned mass damper, Mech Syst Signal Process, 60-61 437-51, 2015.

Mukherjee D, Rao B N, Prasad A M, Global sensitivity analysis of unreinforced masonry structure using high dimensional model representation, Eng Struct, 33 1316-25, 2011.

Mukhopadhyay T, Adhikari S, Effective in-plane elastic moduli of quasi-random spatially irregular hexagonal lattices, International Journal of Engineering Science, 119 142-179, 2017a. 
Mukhopadhyay T, Adhikari S, Equivalent in-plane elastic properties of irregular honeycombs: An analytical approach, International Journal of Solids and Structures, 91 169-184, 2016a.

Mukhopadhyay T, Adhikari S, Stochastic mechanics of metamaterials, Composite Structures, 162 85-97, 2017b.

Mukhopadhyay T. (2017). Mechanics of quasi-periodic lattices, PhD thesis, Swansea University

Mukhopadhyay T. (2019) A multivariate adaptive regression splines based damage identification methodology for web core composite bridges including the effect of noise, Journal of Sandwich Structures \& Materials, DOI: 10.1177/1099636216682533

Mukhopadhyay T., Mahata A., Dey S., Adhikari S. (2016b) Probabilistic analysis and design of HCP nanowires: an efficient surrogate based molecular dynamics simulation approach, Journal of Materials Science \& Technology, 32(12) 1345-1351

Mukhopadhyay T., Adhikari S. (2016b) Effective in-plane elastic properties of auxetic honeycombs with spatial irregularity, Mechanics of Materials, 95 204-222

Mukhopadhyay T., Adhikari S. (2016c) Free vibration analysis of sandwich panels with randomly irregular honeycomb core, Journal of Engineering Mechanics, 142(11) 06016008

Mukhopadhyay T., Adhikari S., Batou A. (2018a) Frequency domain homogenization for the viscoelastic properties of spatially correlated quasi-periodic lattices, International Journal of Mechanical Sciences, DOI: 10.1016/j.ijmecsci.2017.09.004

Mukhopadhyay T., Chowdhury R., Chakrabarti A. (2016a) Structural damage identification: A random sampling-high dimensional model representation approach, Advances in Structural Engineering, 19(6) 908-927

Mukhopadhyay T., Dey T. K.,Chowdhury R., Chakrabarti A., Adhikari S. (2015) Optimum design of FRP bridge deck: an efficient RS-HDMR based approach, Structural and Multidisciplinary Optimization, 52 (3) 459-477

Mukhopadhyay T., Mahata A., Adhikari S., Asle Zaeem M. (2017b) Effective elastic properties of two dimensional multiplanar hexagonal nano-structures, 2D Materials, 4025006

Mukhopadhyay T., Mahata A., Adhikari S., Asle Zaeem M. (2018b) Probing the shear modulus of twodimensional multiplanar nanostructures and heterostructures, Nanoscale, 105280 - 5294

Mukhopadhyay T., Mahata A., Adhikari S., Asle Zaeem M. (2017a) Effective mechanical properties of multilayer nano-heterostructures, Scientific Reports, 715818

Mukhopadhyay T., Naskar S., Dey S., Adhikari S. (2016c) On quantifying the effect of noise in surrogate based stochastic free vibration analysis of laminated composite shallow shells, Composite Structures, 140 798-805

Naskar S, Mukhopadhyay T, Sriramula S, Adhikari, Stochastic natural frequency analysis of damaged thin-walled laminated composite beams with uncertainty in micromechanical properties, Composite Structure, $160312-334,2017 \mathrm{a}$ 
Naskar, S. \& Sriramula, S. (2017a). Random Field Based Approach for Quantifying the Spatial Variability in Composite Laminates. Paper presented at 20th International Conference on Composite Structures (ICCS20), Paris, France, 4/09/17 - 7/09/17.

Naskar, S. \& Sriramula, S. (2017b). Vibration analysis of hollow circular laminated composite beams - A stochastic approach. Paper presented at 12th International Conference on Structural Safety \& Reliability, Vienna, Austria, 6/08/17 - 10/08/17.

Naskar, S., Mukhopadhyay, T. \& Sriramula, S. (2017b). Non-probabilistic analysis of laminated composites based on fuzzy uncertainty quantification. Paper presented at 20th International Conference on Composite Structures (ICCS20), Paris, France, 4/09/17 - 7/09/17.

Naskar, S. (2017). 'Effective Elastic Property of Randomly Damaged Composite Laminates'. Engineering Postgraduate Research Symposium, Aberdeen, United Kingdom, 26/04/17 - 26/04/17

Naskar, S. \& Sriramula, S. (2017c) On Quantifying the Effect of Noise in Radial Basis Based Stochastic Free Vibration Analysis of Laminated Composite Beam, 18 European Conference on Composite Materials, Athens, Greece, 25/06/2018-28/06/2018

Neves A M A, Ferreira A J M, Free vibrations and buckling analysis of laminated plates by oscillatory radial basis functions, Curved and Layered Structures, 3(1) 17-21, 2016.

Niederreiter H, Random number generation and quasi-Monte Carlo methods, CBMS-NSF regional conference series in applied mathematics. Society for Industrial and Applied Mathematics, vol. 63, 1992.

Oberkampf W L, Helton J C, Sentz K, Mathematical representation of uncertainty, In: 42nd AIAA/ASME/ASCE/AHS/ASC structures, structural dynamics, and materials conference and exhibit, Seattle, WA, 2001.

Patel, S. N., \& Sheikh, A. H. (2016). Buckling response of laminated composite stiffened plates subjected to partial in-plane edge loading. International Journal for Computational Methods in Engineering Science and Mechanics, 17(5-6), 322-338

Pawar P M, Nam Jung S., Ronge B P, Fuzzy approach for uncertainty analysis of thin walled composite beams, Aircraft Engineering and Aerospace Technology, 84 (1) 13-22, 2012.

Qatu M S, Leissa A W, Natural frequencies for cantilevered doubly-curved laminated composite shallow shells, Composite Structures, 17 227-55, 1991.

Rabitz H, Alıs O F, General foundations of high-dimensional model representations, J Math Chem, 25 197-233, 1999.

Rayleigh J W, Theory of Sound, Dover Publications, New York, re-issue, second edition, 1945.

Sakata S, Ashida F, Zako M, Kriging-based approximate stochastic homogenization analysis for composite materials, Comput Methods Appl Mech Eng, 197(21) 1953-64, 2008.

Scarth, C, Adhikari, S, Modelling spatially varying uncertainty in composite structures using lamination parameters, AIAA Journal, 55(11) 3951-3965, 2017. 
Sobol I M, On the distribution of points in a cube and the approximate evaluation of integrals, USSR Comput Math Math Phys, 7 86-112, 1967.

Soden P D, Hinton M J, Kaddour A S, Lamina properties, lay-up configurations and loading conditions for a range of fibre reinforced composite laminates, Compos. Sci. Technol., 58 (7) 1011-1022, 1998.

Sofiyev A. H., Hui D., Haciyev V. C., Erdem H., Yuan G. Q., Schnack E., Guldal V. , The nonlinear vibration of orthotropic functionally graded cylindrical shells surrounded by an elastic foundation within first order shear deformation theory. Composites Part B: Engineering, 116;170-185, 2017.

Sofiyev A.H., Najafov A.M., Kuruoğlu N., The effect of non-homogeneity on the non-linear buckling behavior of laminated orthotropic conical shells. Composites Part B: Engineering, 43, 1196-1206, 2012.

Sofiyev, A. H., Kuruoglu N., Combined influences of shear deformation, rotary inertia and heterogeneity on the frequencies of cross-ply laminated orthotropic cylindrical shells. Composites Part B: Engineering, $66,500-510,2014$.

Sofiyev, A. H., \& Kuruoglu, N., Determination of the excitation frequencies of laminated orthotropic non-homogeneous conical shells. Composites Part B: Engineering, 132, 151-160, 2018

Sriramula S, Chryssanthopoulos K M, Quantification of uncertainty modelling in stochastic analysis of FRP composites, Composites Part A: Applied Science and Manufacturing, 40 1673-1684, 2009

Sudret B, Der Kiureghian A, Stochastic Finite Element Methods and Reliability - A State-of-the-Art Report, Tech. Rep. UCB/SEMM- 2000/08, Department of Civil \& Environmental Engineering, Univ. of California, Berkeley, Nov. 2000.

Talha Md, Singh B N, Stochastic perturbation-based finite element for buckling statistics of FGM plates with uncertain material properties in thermal environments, Compos Struct, 108 823-33, 2014.

Umesh K, Ganguli R, Material uncertainty effect on vibration control of smart composite plate using polynomial chaos expansion, Mech Adv Mater Struct, 20 (7) 580-591, 2013.

Vanmarcke E, Grigoriu M, Stochastic finite-element analysis of simple beams, Journal of Engineering Mechanics-ASCE, 109 (5) 1203-1214, 1983.

Vanmarcke E, Random fields: analysis and synthesis, World Scientific Publishing Co. Pte Ltd., Cambridge, 1983.

Zhang J, Ellingwood B, Orthogonal series expansion of random fields in reliability analysis, Journal of Engineering Mechanics-ASCE, 120 (12) 2660-2677, 1994.

Ziehn T, Tomlin A S, GUI-HDMR - A software tool for global sensitivity analysis of complex models, Environ Model Softw, 24 (7) 775-85, 2009. 